\title{
Our astrochemical heritage
}

\section{Paola Caselli · Cecilia Ceccarelli}

Received: 3 September 2012 / Accepted: 7 October 2012

\begin{abstract}
Our Sun and planetary system were born about 4.5 billion years ago. How did this happen and what is our heritage from these early times? This review tries to address these questions from an astrochemical point of view. On the one hand, we have some crucial information from meteorites, comets and other small bodies of the Solar System. On the other hand, we have the results of studies on the formation process of Sun-like stars in our Galaxy. These results tell us that Sun-like stars form in dense regions of molecular clouds and that three major steps are involved before the planet formation period. They are represented by the pre-stellar core, protostellar envelope and protoplanetary disk phases. Simultaneously with the evolution from one phase to the other, the chemical composition gains increasing complexity.

In this review, we first present the information on the chemical composition of meteorites, comets and other small bodies of the Solar System, which is potentially linked to the first phases of the Solar System's formation. Then we describe the observed chemical composition in the pre-stellar core, protostellar envelope and protoplanetary disk phases, including the processes that lead to them. Finally, we draw together pieces from the different objects and phases to understand whether and how much we inherited chemically from the time of the Sun's birth.
\end{abstract}

P. Caselli

School of Physics and Astronomy, University of Leeds, Leeds LS2 9JT, UK

Tel.: +44-(0)113-3434065

Fax: +44-(0)113-3433900

E-mail: P.Caselli@leeds.ac.uk

C. Ceccarelli

UJF-Grenoble 1/CNRS-INSU, Institut de Planétologie et d'Astrophysique de Grenoble (IPAG) UMR 5274, Grenoble, F-38041, France

Tel.: +33-(0)476-514201

Fax: +33-(0)476-448821

E-mail: Cecilia.Ceccarelli@obs.ujf-grenoble.fr 
Keywords Astrochemistry - ISM: clouds - Stars: formation - Protoplanetary disks · Comets: general · Meteorites, meteors, meteoroids 


\section{Introduction}

Once upon a time, there was a small cold cloud of gas and dust in an interstellar medium broken into several clumps and filaments of different masses and dimensions. Then, about 4.5 billion years ago, the small cloud became the Solar System. What happened to that primordial cloud? When, why and how did it happen? Does the Earth receive a heritage from those old eons? Can this heritage help us to understand our origins?

The answers to these questions can only come from putting together many pieces of a giant puzzle that covers different research fields: from what the Earth is made of to its evolution, from what are the most pristine meteorites from outer space that have fallen on Earth to their present composition, from which other small bodies of the Solar System, comets and asteroids, have the imprint of the first composition of the solar nebula to their origin and evolution. Last but not least, the study of other small clouds and young Sunlike stars in our Galaxy gives us the wide range of possible outcomes of star and planet formation, from which we would like to understand why the Solar System and the Earth chose one of them.

Each single piece of the puzzle brings precise and precious information. The problem is that sometimes the information is hidden in a scrambled code whose key is unknown. Take meteorites as an example. As explained to us by an expert colleague, assessing the composition of the Solar Nebula from the study of the meteorites is like trying to assess Napoleon's army structure looking at the few survivors of the Russian war. How representative are those survivors? Although, evidently, they still provide very precious information, extracting the whole information from them is far from obvious. The example is to say that every single piece of the puzzle is important, even the pieces that seem to be redundant. Actually, the redundant ones are likely the most important, as they may allow to distinguish and disentangle all the various intervening effects. In this context, the study of the objects similar to the Solar System progenitor takes a particular relevance, because it can provide us with plenty of pieces to compare with the other pieces from the present Solar System. The hope is that they will provide us with the keys of the scrambled codes.

In this review, we will focus on just a subset of these pieces, those coming from the study of the chemical composition during the birth of stars and planetary systems like our Solar System. In \$2, we will first give a very general overview of how we think the Solar System and stars of similar mass have formed and how this process influences the chemistry. This is based on the ensemble of observations and studies on star-forming regions and Solar System objects. Then, in $\$ 3$ we will describe in detail some pieces of the puzzle which potentially connect what we observe in the objects of the Solar System nowadays and what we know about star formation in our Galaxy. The next sections will discuss star and planet formation studies. We will describe how the evolution of the matter from a cold cloud (\$4) to a protostellar envelope (\$5) and a protoplanetary disk ( $\$ 6$ corresponds to an increase of the molecular complexity. Section 7 will provide specific examples on the link between 


\section{FROM A DIFFUSE CLOUD TO A SUN + PLANETARY SYSTEM FROM ATOMS \& SIMPLE MOLECULES TO LIFE}

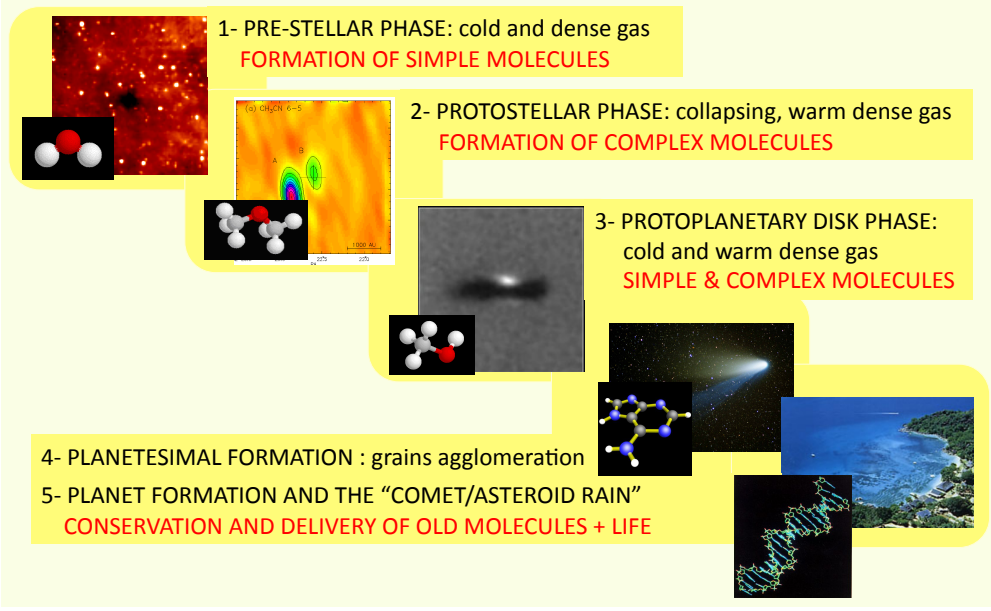

Fig. 1 Star formation and chemical complexity. The formation of a star and a planetary system, like the Solar System, passes through five fundamental phases, marked in the sketch.

the present Solar System small bodies with the pre- and proto-stellar phase. A final section will try to draw some conclusions.

We emphasize that the present review is complementary to several reviews recently appeared in the literature on different aspects just touched upon by us and that will be cited in the appropriate sections.

\section{Solar-type star formation and chemical complexity}

The formation of a Sun-like star and molecular complexity proceed hand in hand. As the primordial cloud evolves into a protostellar envelope, protoplanetary disk and planetary system, the chemical composition of the gas becomes increasingly more complex. The five major phases of the process that we think have formed the Earth are sketched in Figure 1 and here listed.

Phase 1: Pre-stellar cores. These are the "small cold clouds" mentioned above. During this phase, matter slowly accumulates toward the center of the nebula. As a result, the density at the center increases while the temperature decreases. Atoms and molecules in the gas-phase freeze-out onto the cold surfaces of the sub-micron dust grains, forming the so-called icy grain mantles. Thanks to the mobility of the $\mathrm{H}$ atoms on the grain surfaces, hydrogenation of atoms and CO (the most abundant molecule, after $\mathrm{H}_{2}$, in cold molecular gas) takes place, forming molecules such as water 
$\left(\mathrm{H}_{2} \mathrm{O}\right)$, formaldehyde $\left(\mathrm{H}_{2} \mathrm{CO}\right)$, methanol $\left(\mathrm{CH}_{3} \mathrm{OH}\right)$ and other hydrogenated species.

Phase 2: Protostellar envelopes. The collapse proceeds, gravitational energy is converted into radiation and the envelope around the central object, the future star, warms up. The molecules frozen in grain mantles during the previous phase acquire mobility and likely form new, more complex species. When the temperature reaches the mantle sublimation temperature, in the so-called hot corinos, the molecules in the mantles sublimate back in the gas-phase, where they react and form new, more complex, molecules. Simultaneously to the collapse, a fraction of matter is violently ejected outward in the form of highly supersonic collimated jets and molecular outflows. When the outflowing material encounters the quiescent gas of the envelope and the molecular cloud, it creates shocks, where the grain mantles and refractory grains are (partially) sputtered and vaporized. Once in the gas phase, molecules can be observed via their rotational lines.

Phase 3: Protoplanetary disks. The envelope dissipates with time and eventually only a circumstellar disk remains, also called protoplanetary disk. In the hot regions, close to the central object, new complex molecules are synthesized by reactions between the species formed in the protostellar phase. In the cold regions of the disk, where the vast majority of matter resides, the molecules formed in the protostellar phase freeze-out again onto the grain mantles, where part of the ice from the pre-stellar phase may still be present. The process of "conservation and heritage" begins.

Phase 4: Planetesimal formation. The sub-micron dust grains coagulate into larger rocks, called planetesimals, the seeds of the future planets, comets and asteroids. Some of the icy grain mantles are likely preserved while the grains glue together. At least part of the previous chemical history may be conserved in the building blocks of the Solar System rocky bodies.

Phase 5: Planet formation. This is the last phase of rocky planet formation, with the embryos giant impact period and the formation of the Moon and Earth. The leftovers of the process, comets and asteroids, copiously "rain" on the primitive Earth, forming the oceans and the Earth second atmosphere. The heritage conserved in the ices trapped in the planetesimals and rocks is released onto the Earth. Life emerges sometime around 2 billion years after the Earth and Moon formation 1

The sections 4 to 6 will review and discuss in detail the chemistry in the first three phases of the process, those where the heritage is likely accumulated. Box 1 briefly explains the data and tools needed to interpret the observations and Table 1 summarizes some key proprieties of the phase 1 to phase 3 objects.

\footnotetext{
1 The famous fossils of cyanobacteries of Australia and for long considered as the first traces of life dated 3.5 Myr ([393), are interpreted as inorganic condensations ([403]; 167]) and still source of intense debate (284]). Conversely, there is consensus on the rise of life about $2 \mathrm{Gyr}$ after the Earth formation, as testified by the rise in the $\mathrm{O}_{2}$ abundance in the atmosphere (121]).
} 
Table 1 Summary of the proprieties of the objects in the first three phases of the solar-type star formation process, before planet formation.

\begin{tabular}{|c|c|c|c|c|c|}
\hline Phase \& Object & $\begin{array}{l}\text { Age } \\
(\mathrm{yr})\end{array}$ & $\begin{array}{l}\text { Radius } \\
\text { (AU) }\end{array}$ & $\begin{array}{r}\text { Temp. } \\
(K)\end{array}$ & $\begin{array}{l}\text { Density } \\
\left(\mathrm{cm}^{-3}\right)\end{array}$ & Chemical processes \\
\hline 1- Pre-stellar core & $\sim 10^{5}$ & $\sim 10^{4}$ & $7-15$ & $10^{4}-10^{6}$ & $\begin{array}{l}\text { Ice formation \& } \\
\text { molecular deuteration }\end{array}$ \\
\hline 2- Protostellar envelope: & $10^{4}-10^{5}$ & $\sim 10^{4}$ & & & \\
\hline Cold envelope & & $100-10^{4}$ & $\leq 100$ & $10^{5}-10^{7}$ & $\begin{array}{l}\text { Ice formation \& } \\
\text { molecular deuteration }\end{array}$ \\
\hline Hot corinos & & $\leq 100$ & $\geq 100$ & $\geq 10^{7}$ & $\begin{array}{l}\text { Complex molecules } \\
\text { formation }\end{array}$ \\
\hline 3- Protoplanetary disk: & $\sim 10^{6}$ & $\sim 200$ & & & \\
\hline Outer midplane & & $20-200$ & $100-10$ & $10^{8}-10^{6}$ & $\begin{array}{l}\text { Ice formation \& } \\
\text { molecular deuteration }\end{array}$ \\
\hline Inner midplane & & $\leq 20$ & $\geq 100$ & $\geq 10^{8}$ & $\begin{array}{l}\text { Complex molecules } \\
\text { formation }\end{array}$ \\
\hline
\end{tabular}

Box 1: Data needed to interpret the astronomical observations In order to derive the chemical composition of a celestial body from line observations one needs to identify correctly the lines as due to a specific molecule and to convert the observed line intensity into the species abundance. Then, to understand what these abundances mean one needs to compare the observed with model predicted abundances. The process, sketched in Fig.2 requires, therefore, data from different communities: i) spectroscopic data, to identify the lines; ii) collisional coefficients, to convert them into abundances; iii) chemical reactions to build up astrochemical models. The three sets of data necessitate specific skills and enormous laboratory and computational efforts. The available information is centralised in the following databases:

- Spectroscopic databases:

+ JPL Molecular spectroscopy database ([348]):

http://spec.jpl.nasa.gov/home.html

+ Cologne Database for Molecular Spectroscopy database (CDMS, 308):

http://www.astro.uni-koeln.de/cdms/

+ Splatalogue database for astronomical spectroscopy (SPLATALOGUE):

http://splatalogue.net/

- Collisional excitation databases:

+ Ro-vibrational collisional excitation database (BASECOL, 134):

http://basecol.obspm.fr/index.php?page=pages/generalPages/home

+ Leiden Atomic and Molecular Database (LAMDA, [392]):

http://home.strw.leidenuniv.nl/ moldata/

- Chemical reaction databases:

+ The KInetic Database for Astrochemistry (KIDA, 445]):

http://kida.obs.u-bordeaux1.fr/

+ The Ohio State University (OSU) gas-phase and gas-grain chemical models (e.g. [172]):

http://www.physics.ohio-state.edu/ eric/research.html

+ The UMIST database for astrochemistry (UDFA, [469]):

http://www.udfa.net/

We emphasize that the databases just collect the data which are provided by several colleagues from all over the world. We would like here to pay our tribute to Pierre Valiron and Fredrik Schoier, who enormously contributed to the collisional excitation coefficients and to the set up of the LAMDA database respectively, and who prematurely passed away. 


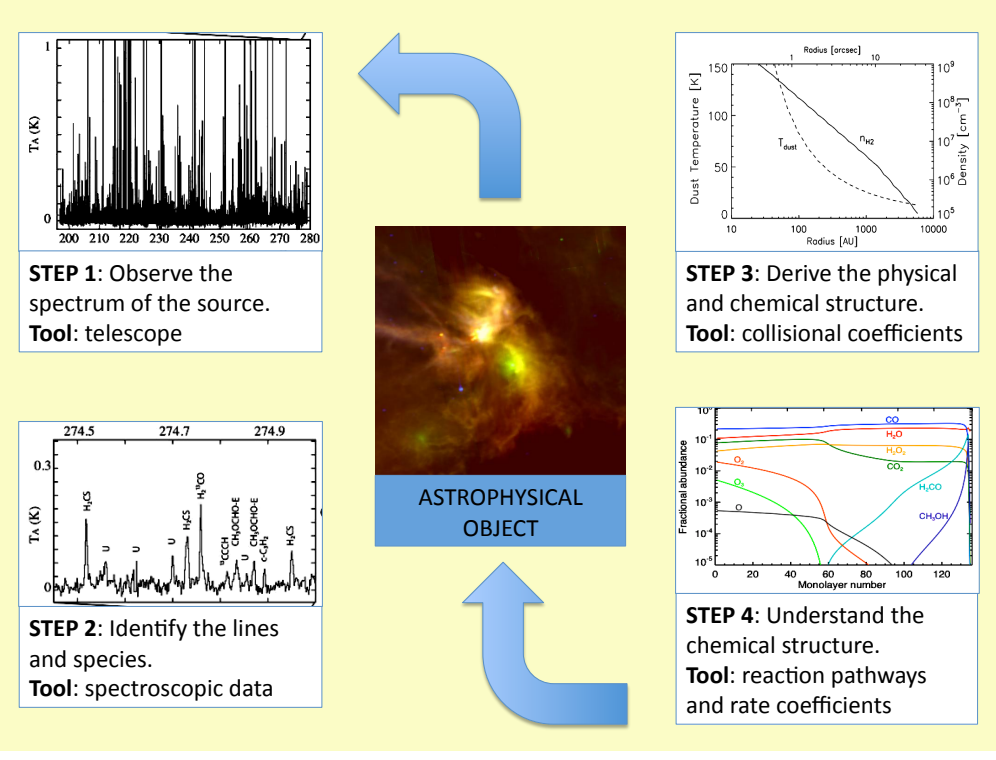

Fig. 2 The four steps required to measure the chemical structure of an astrophysical object, as described in Box 1, including the tools needed to complete each step: (1) observations at the telescope, (2) identification of the lines and species, (3) derivation of the physical and chemical structure using radiative transfer codes, which require accurate collisional coefficients, and (4) chemical models.

\section{Pieces of the puzzle from the Solar System}

A variety of information on the formation process of the Solar System is provided to us by the small bodies believed to be the most pristine objects of the Solar System: Kuiper Belt Objects (KBOs), comets, meteorites and particularly carbonaceous chondrites, and interplanetary dust particles (IDPs). Here we will review some properties of these objects that can shed light on the formation process when compared with what we know about other solartype forming stars in the Galaxy. We emphasize that this summary is far from being exhaustive and the reader is invited to look at the reviews cited in the following subsections.

3.1 Where does the terrestrial water come from?

We all know how fundamental water is for the terrestrial life. It is the best solvent, allowing chemical reactions to form large biotic molecules and to break down ATP (Adenosine TriPhosphate), a process at the very base of the energy metabolism of living cells. Water had a fundamental role also on planet Earth, 
its history, evolution and equilibrium, for example allowing the magma to be viscous enough for tectonics to take place.

Sometimes the most obvious questions, like the one on the origin of water or why the night sky is dark, do not have obvious answers. The explanation of the dark night sky had to wait for the discovery of the expansion of the Universe, while the explanation of why Earth is so abundant in water is still hotly debated. But what are the facts? Two main facts are fundamental pieces of this puzzle. The first one is the quantity of terrestrial water, the second is its isotopic composition.

Regarding the amount of water on Earth, we can easily measure it in the Earth's crust where it is $\sim 3 \times 10^{-4}$ the Earth mass (260). It is much less obvious to measure it in the mantle and core, where the vast majority of the Earth's mass resides and where it is impossible to directly measure the volatile components. Measurements of Earth's mantle water content are in fact based on indirect evidence, mostly using noble gases as proxies for the volatile hydrogen (152; 10]), which implies assuming that the solar abundance ratios are maintained in the Earth mantle. The most recent estimates give a total amount of $\sim 2 \times 10^{-3}$ Earth masses (285]), namely almost ten times more than in the crust. It has to be noted, though, that Earth in the Archaean was most likely more volatile-rich than in our days (e.g. 241).

The second fundamental piece of the puzzle is the $\mathrm{HDO} / \mathrm{H}_{2} \mathrm{O}$ ratio, $1.5 \times$ $10^{-4}$ in the terrestrial oceans, namely about ten times larger than the elemental D/H ratio in the Solar Nebula ([174]). Direct measurements of the $\mathrm{HDO} / \mathrm{H}_{2} \mathrm{O}$ ratio in the Earth mantle are impossible, but indirect ones seem to suggest a slightly lower value than that of the oceans (285]).

The problem on the origin of the terrestrial water comes from the fact that the planetesimals that built up the Earth, if they were located at the same place where Earth is today, must have been dry. Therefore, either water came later, when Earth was mostly formed, or the planetesimals that formed the Earth were from a zone more distant than 1 AU. The first theory, also called "late veneer", was first proposed by [129] and 332 and postulates that water is mostly delivered to Earth from comets, especially during the Late Heavy Bombardment ([128; [180). For almost a decade, the theory had the problem, though, that the $\mathrm{HDO} / \mathrm{H}_{2} \mathrm{O}$ abundance ratio in the six comets where it had been measured is about a factor of two too high (224]; see 3.4 and Fig. 4). However, new Herschel measurements are changing the situation. The measure on the 103P/Hartley2 comet gives exactly the terrestrial value ([195]) whereas measurements toward $\mathrm{C} / 2009 \mathrm{P} 1$ give again a larger $\mathrm{HDO} / \mathrm{H}_{2} \mathrm{O}$ value, $2 \times 10^{-4}([51])$. The other possibility is that Earth was partly built from water-rich planetesimals from the outer zone ([303]). Two arguments are in favor of this theory. First, the $\mathrm{HDO} / \mathrm{H}_{2} \mathrm{O}$ ratio of carbonaceous chondrites is very similar to the terrestrial one $\left(1.3-1.8 \times 10^{-4}, 375\right]$; see $\$ 3.4$ and Fig. 44). Second, numerical simulations of the young Solar System from several authors predict that up to $10 \%$ of the Earth may have been formed by planetesimals from the outer asteroid belt, providing enough water to Earth (e.g. 303; [367]). The same simulations tend to exclude the cometary delivery as a major 
contribution. However, as any model, the predictions are subject to a number of uncertainties, a major one being how much water is in the outer asteroid belt planetesimals $([269])$.

Finally, the question on the origin of Earth's water is somewhat linked to the question on the origin of the Earth's atmosphere. Even though the methods are different, also for the Earth's atmosphere it is discussed a cometary delivery versus a meteoritic origin. Likely, in this case, both sources are necessary (e.g. [126]).

We emphasize the key role played, in both theories, by the $\mathrm{HDO} / \mathrm{H}_{2} \mathrm{O}$ ratio in the terrestrial water, comets and asteroids. In the following sections of this review, we will see why, when and how water becomes enriched of deuterium.

\subsection{Molecular species in comets and KBOs}

Several molecular species have been detected in comets since decades and in KBOs since the last decade. Here we briefly summarise which species have been detected and recommend to the interested reader the reviews by 309. and [49], and 69]. on the comets and KBOs, respectively.

Comets: Two dozens of molecular species have been identified in various comets by several authors (e.g. [43]; 118]). More specifically:

i) $\mathrm{H}_{2} \mathrm{O}, \mathrm{CO}, \mathrm{CO}_{2}, \mathrm{CH}_{4}, \mathrm{C}_{2} \mathrm{H}_{2}, \mathrm{C}_{2} \mathrm{H}_{6}, \mathrm{CH}_{3} \mathrm{OH}, \mathrm{H}_{2} \mathrm{CO}, \mathrm{NH}_{3}, \mathrm{HCN}, \mathrm{HNC}$, $\mathrm{CH}_{3} \mathrm{CN}$ and $\mathrm{H}_{2} \mathrm{~S}$ have been detected in more than 10 comets;

ii) $\mathrm{HCOOH}, \mathrm{HNCO}, \mathrm{HC}_{3} \mathrm{~N}$, OCS and $\mathrm{S}_{2}$ have been detected in more than 1 comet;

iii) $\mathrm{HOCH}_{2} \mathrm{CH}_{2} \mathrm{OH}, \mathrm{HCOOCH}_{3}, \mathrm{CH}_{3} \mathrm{CHO}, \mathrm{NH}_{2} \mathrm{CHO}, \mathrm{SO}_{2}, \mathrm{H}_{2} \mathrm{CS}$ have been observed in one comet, Hale-Bopp.

Not all species are considered primary species, namely species present in the sublimated ices. Some, like HNC, are product species, namely they are the products of chemical reactions involving the primary species once ejected in the gas. Other species, like $\mathrm{H}_{2} \mathrm{CO}$ and $\mathrm{CO}$, have contributions from both primary and product species. The measured abundances are summarised in Fig. 3. To this list one has to add the recent detection of glycine, the simplest of amino acids, in the $81 \mathrm{P} /$ Wild2 comet by the mission STARDUST ([146]).

KBOs: KBOs are the objects beyond Neptun's orbit, at an heliocentric distance between 30 and $50 \mathrm{AU}$, and are thought to hold precious information on the pristine chemical composition of the Solar Nebula at those distances. Being relatively small objects, they are difficult to study. However, in the last decade, important progress has been made. Briefly, the six large KBOs where spectroscopic observations could be obtained showed the presence in their atmosphere of $\mathrm{H}_{2} \mathrm{O}, \mathrm{CH}_{4}, \mathrm{~N}_{2}$ and $\mathrm{CO}$, even though with different proportions from object to object (e.g. [26] [390; 70]). In addition, ethane $\left(\mathrm{C}_{2} \mathrm{H}_{6}\right)$, believed to be the result of $\mathrm{CH}_{4}$ photolysis processes caused by the solar wind and cosmic rays, has been detected in Makemake ([33]). In other smaller KBOs, spectroscopic 


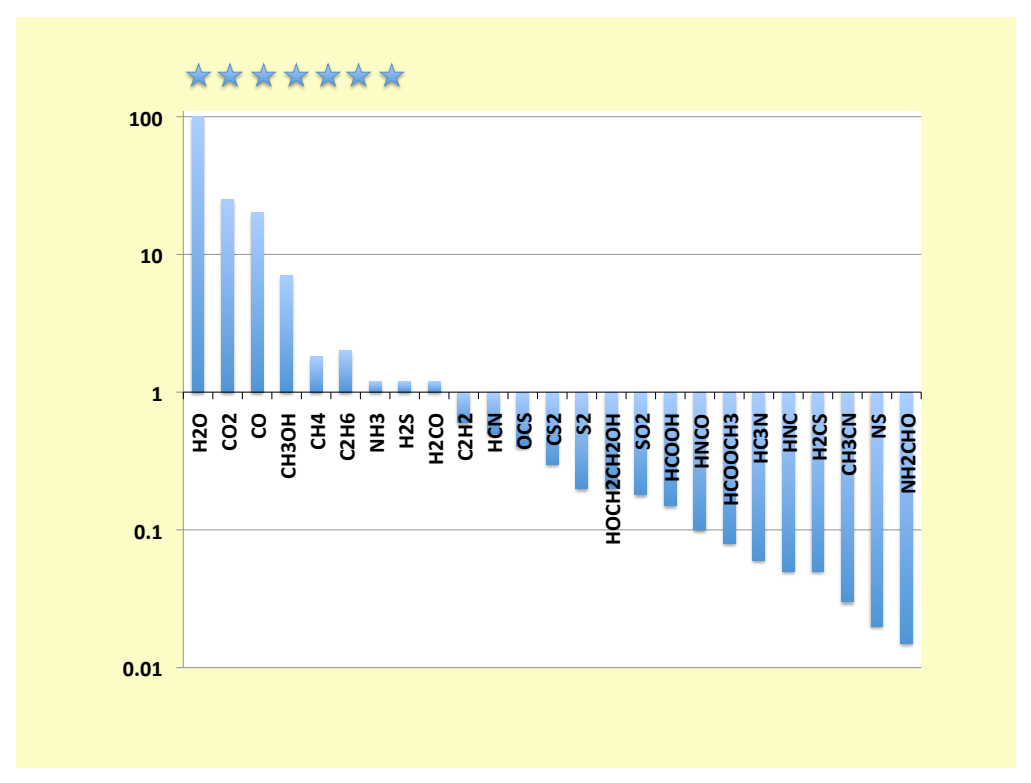

Fig. 3 Abundances of molecular species in comets, with respect to $\mathrm{H}_{2} \mathrm{O}$. The species with an asterisk have also been detected in KBOs.

observations showed the presence of water, ammonia and likely methanol ices ([27; ; 70]).

From Fig. 3 , it is clear that the most abundant species in comets $\left(\mathrm{H}_{2} \mathrm{O}\right.$, $\mathrm{CO}, \mathrm{CO}_{2}, \mathrm{CH}_{4}, \mathrm{NH}_{3}, \mathrm{CH}_{3} \mathrm{OH}$ and $\mathrm{C}_{2} \mathrm{H}_{6}$ ) are observed also in $\mathrm{KBOs}$ and, in turn, the species observed in KBOs are the most abundant species in comets. Formaldehyde and hydrogen sulfide have abundances in comets comparable to $\mathrm{CH}_{4}$ and $\mathrm{NH}_{3}$. Their non detection in KBOs may, however, be due to observational effects only.

\subsection{Organics in meteorites and IDPs}

Carbonaceous chondrites are rich in carbon, which constitutes about $1-4 \%$ of this kind of meteorites. Organic carbon is present in two forms, following the methods to extract the organic material: insoluble organic matter (IOM) and soluble organic matter $(\mathrm{SOM})$.

IOM is mainly $(\geq 70 \%)$ constituted of organic compounds with a relatively complex structure (nanoglobules, venatures...). The compounds are made of small aromatic units (with up to six rings) linked by branched aliphatic linkages shorter than seven carbon atoms (e.g. [370]; 259]). Similarly, IDPs contain about $10-12 \%$ carbon, mostly in organic material, including aromatic and aliphatic compounds ([420; [243]). 
SOM is principally made of carboxylic acids, aliphatic and aromatic hydrocarbons, and amino acids (e.g. 354]). In the Murchison meteorite, they represent $\sim 50 \%, \sim 25 \%$ and $\sim 10 \%$, respectively, of the organic soluble matter. Of particular interest, amino acids with no known terrestrial distribution have been found in meteorites. In addition, a sub-group of amino acids shows a small but significant L-enantiomeric excesses (e.g. 355]), namely one of the two chiral forms is more abundant than the other, a characteristic of chiral biomolecules in terrestrial life.

\subsection{The hydrogen and nitrogen isotopic anomalies}

One direct evidence of the link between pristine small Solar System bodies like carbonaceous chondrite, IDPs and comets, and the first phases of the Sun formation (phases 1 to 3 in Fig. 1) comes from the presence of the so-called isotopic anomalies. Among the five most abundant elements in the Universe $(\mathrm{H}, \mathrm{He}, \mathrm{O}, \mathrm{C}$ and $\mathrm{N})$, three present large anomalies, namely they have isotopic values more than twice different than in the Solar Nebula: hydrogen, oxygen and nitrogen (while carbon also shows different values but to a lesser extent). Each of them brings different information. Here, we briefly review the information provided by the hydrogen and nitrogen isotopes. Oxygen isotopic anomalies are discussed in 3.5 .

Deuterium in comets: The first and most important isotopic anomaly, the deuterium enrichment of terrestrial water, has been already discussed in 33.1 . We also briefly mentioned that comets show a D-enrichment one to two times the one of the terrestrial oceans (Fig. 4). Regardless whether comets substantially contributed or not to the terrestrial water, the relatively high abundance of deuterated water can help us to understand when and where comets formed and, consequently, how the Solar System formed. So far, the $\mathrm{HDO} / \mathrm{H}_{2} \mathrm{O}$ ratio has been observed in seven comets from the Oort Cloud, the most recent being the $\mathrm{C} / 2009 \mathrm{P} 1$ comet, and in one, 103P/Hartley2, from the Jupiter family comets. In the first six comets, $\mathrm{HDO} / \mathrm{H}_{2} \mathrm{O}$ has been measured to be $\sim 3 \times 10^{-4}$ ([307]; [52]), in C/2009 P1 it is $2 \times 10^{-4}$ ([51]), and in 103P/Hartley2 it is $\left.1.5 \times 10^{-4}(195]\right)$. If, on the one hand, this last measurement has brought back to life the debated late veneer theory (3.1), it has also challenged the present view of where these comets are formed. In fact, according to the widely-accepted theory, comets from the Oort Cloud and the Jupiter family were likely formed in the Uranus-Neptune zone ([132]), even though the Oort Cloud comets may also originate from the Jupiter-Saturn region ([66]). The $\mathrm{HDO} / \mathrm{H}_{2} \mathrm{O}$ ratio is an almost direct measure of the temperature where the comet is formed and larger heliocentric distances are expected to correspond to colder regions. Therefore, one would expect that comets in the Oort Cloud present a similar or lower $\mathrm{HDO} / \mathrm{H}_{2} \mathrm{O}$ ratio than the Jupiter family comets, contrary to what is measured. Dedicated models support this simple intuitive argument (e.g. 214]; 240; 347]). Therefore, either comet formation theory 
is not correct in this aspect (for example a new theory postulates that Oort Cloud comets are captured from nearby stars; 268), or the temperature in the Solar Nebula was not monotonically decreasing with increasing heliocentric distance. This is in principle possible during the accreting disk phase where viscosity may have created warm regions (e.g. 478). This will be further discussed in \$7. So far for water, but deuterium enrichment is also observed in $\mathrm{HCN}$, in one comet (294]), and it is about 10 times larger than the water Denrichment. This difference is not necessarily a problem as it may just outline the different chemical formation pathway of these two species, as explained in $\$ 4.2 .1$.

Deuterium in carbonaceous chondrites and IDPs: The bulk of carbonaceous chondrites contains hydrated silicates and hydrous carbon with a $\mathrm{D} / \mathrm{H}$ ratio $=$ $1.2-2.2 \times 10^{-4}$ (e.g. 375$]$ ), very similar to that of the terrestrial oceans. However, D-enrichment, similar to that measured in comets and even higher, has also been found in the so called "hot spots", namely micrometer-scale regions with positive isotope anomalies, in the IOM of chondrites and IDPs. These hot spots are in fact so named because of the enrichment of D and ${ }^{15} \mathrm{~N}$, and are systematically found in small regions of organic material. The D-enrichment in carbonaceous chondrites and IDPs is very variable, with regions having $\mathrm{D} / \mathrm{H} \sim 8 \times 10^{-5}$ close to the Solar Nebula value, and others having $\mathrm{D} / \mathrm{H}$ up to $\sim 10^{-2}$ (9]; 371]). High spatial resolution measurements suggest that the largest D-enrichment is associated with organic radicals (371]). Similarly, molecules in the soluble organic matter component show enhanced abundances of D-species with respect to $\mathrm{H}$-species, at a level of $\mathrm{D} / \mathrm{H}$ up to almost $10^{-2}$ ([353).

${ }^{15} N$ in comets: Several measurements of the bulk of the ${ }^{14} \mathrm{~N} /{ }^{15} \mathrm{~N}$ in the Solar Nebula, from observations of $\mathrm{NH}_{3}$ in Jupiter ([333; ; 155]) and the solar wind ([286]) give a value of $\sim 440$, consistent with standard stellar nucleosynthesis models. In comets, though, the ${ }^{14} \mathrm{~N} /{ }^{15} \mathrm{~N}$ ratio measured in $\mathrm{CN}$ and $\mathrm{HCN}$ species is more than a factor 2 lower, around 150 (19; ; 279). The origin of this ${ }^{15} \mathrm{~N}$ enrichment in comets has puzzled astrochemists for years. One possibility is that this is a direct heritage of the pre-stellar core phase $(\sqrt{4})$ or proto-planetary disk phase ( $(6)$. Finally, it is also possible that ${ }^{15} \mathrm{~N}$ has been injected in the material forming the Solar System by the explosion of a nearby supernova $(3.5$.

${ }^{15} \mathrm{~N}$ in chondrites and IDPs: The ${ }^{14} \mathrm{~N} /{ }^{15} \mathrm{~N}$ as measured in TiN in a pristine condensate $\mathrm{Ca}-\mathrm{Al}$-rich inclusion of a carbonaceous chondrites is very similar to the Solar Nebula value $(\sim 440,[293])$. However, the ${ }^{14} \mathrm{~N} /{ }^{15} \mathrm{~N}$ in the IOM material of carbonaceous chondrites and IDPs is low, up to $\sim 50$ ([58; [57; [288]), as low as in comets and significantly lower than in the Solar Nebula and the interstellar medium. Similar ${ }^{15} \mathrm{~N}$ enrichment has been reported in two amino acids ([352]). Therefore, the same question on the origin of the ${ }^{15} \mathrm{~N}$ enrichment 


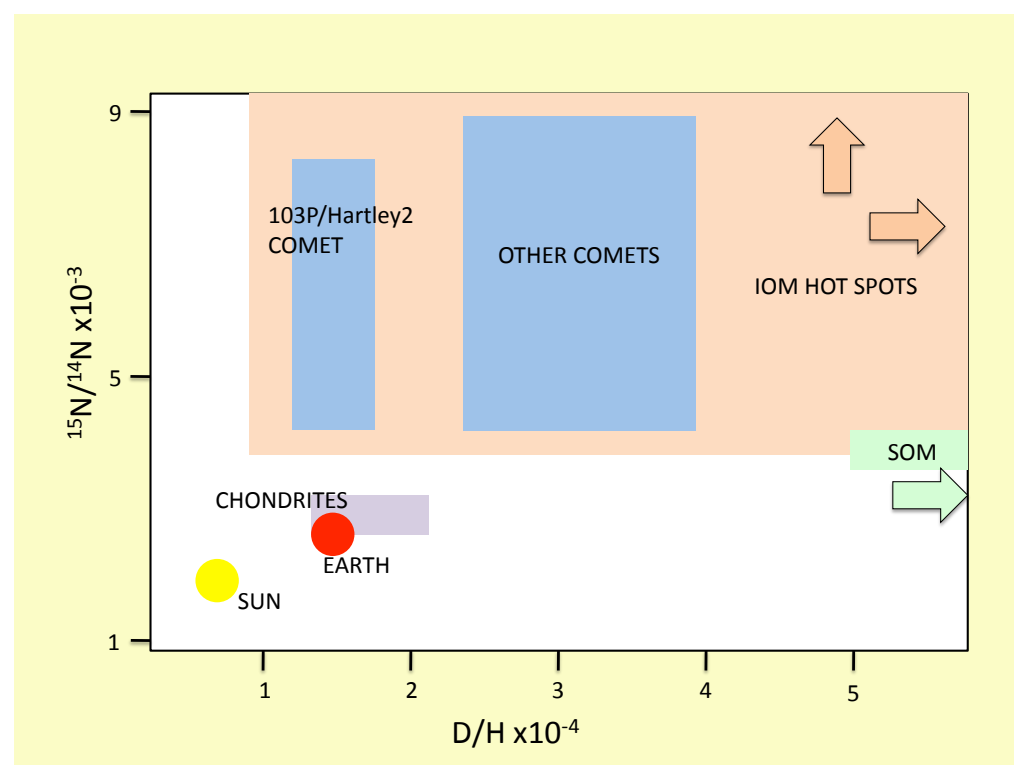

Fig. $4{ }^{15} \mathrm{~N} /{ }^{14} \mathrm{~N}$ versus $\mathrm{D} / \mathrm{H}$ in comets, chondrites, hot spots in the IOM of meteorites and IDPs, SOM in meteorites, Earth and Sun.

in comets applies to the organic material in carbonaceous chondrites and IDPs.

A common origin for the $D$ - and ${ }^{15} N$-enrichment in comets and the organic material in carbonaceous chondrites and IDPs? Since comets and the organic material in chondrites and IDPs are enriched in both $\mathrm{D}$ and ${ }^{15} \mathrm{~N}$, the question whether the enrichment has a common origin is a natural one (e.g. 8] ). Against this hypothesis is that D-enriched spots in chondrites and IDPs do not coincide spatially with ${ }^{15} \mathrm{~N}$-enriched ones $\left.([8] ; 376]\right)$. Similarly, while the D-enrichment differs by a factor two in $103 \mathrm{P} /$ Hartley 2 and the other six comets, the ${ }^{15} \mathrm{~N}$ enrichment is practically the same in all comets (Fig. 4). Therefore, very likely D- and ${ }^{15} \mathrm{~N}$-enrichments do not have a common origin (see also [466]).

\subsection{A violent start in a crowded violent environment}

Short-lived nuclides ${ }^{2}$ present at the formation of the Solar System and now disappeared, and isotopic oxygen anomalies in meteorites tell us that the Solar System had a violent start in a violent environment. First, the young Sun irradiated the forming planetary system with a strong wind of energetic particles. Second, the Sun was likely born in a large cluster of stars where one or more massive stars exploded. All this is based on anomalies with respect to

2 Short-lived nuclides are the radionuclides with half-lives shorter than about $10 \mathrm{Myr}$. 
the "normal" values of the abundances of these elements, which can only be firmly known by assessing what is the normality in other forming stars and, therefore, it is an important piece of the puzzle to mention here.

A violent start: It is now well-known that young solar-type stars are bright X-rays emitters, about $10^{3}$ times brighter than the present day Sun ([151]; [361). It is very likely that, together with X-rays, $\mathrm{H}$ and He nuclei with energies larger than $10 \mathrm{MeV}$ are also emitted in large quantities in the early stages of star formation (e.g. 263). The Sun likely passed through a similar violent phase and irradiated the forming planetary system with energetic particles (sometimes also referred as "early solar cosmic rays"). Extinct short-lived nuclides bring traces of this violent past. Specifically, the enhanced abundances of ${ }^{10} \mathrm{Be},{ }^{7} \mathrm{Li}$ and ${ }^{21} \mathrm{Ne}(292$; 102]) can only be explained by spallation reactions of solar energetic particles with $\mathrm{O}$ and $\mathrm{C}$ atoms of the Solar Nebula. Similarly, other short-lived nuclides, ${ }^{36} \mathrm{Cl},{ }^{53} \mathrm{Mg}$ and ${ }^{41} \mathrm{Ca}$, are now explained in terms of irradiation from the early Sun (e.g. [283]; [185]).

A crowded violent environment: Several lines of evidence converge toward a picture where the Sun was born in a cluster of at least 1000 stars (see the review by [2]). Likely, within this cluster, some were massive stars and some exploded a little before or during the formation of the Solar System. Since its discovery in meteorites, ${ }^{26} \mathrm{Al}$ (247]; 437]) became one of the proofs, indeed highly debated for decades, that the Solar System was polluted with material ejected from a nearby type II supernova, whose progenitor mass is $\sim 25 \mathrm{M}_{\odot}$ ([76] [185]). Support to this hypothesis was added by the discovery of ${ }^{60} \mathrm{Fe}$ ([248), but the value of the ${ }^{60} \mathrm{Fe}$ excess with respect to the Galactic one has been revised since and nowadays it is believed to be close to zero ([305]). As a consequence, theories based on ${ }^{60} \mathrm{Fe}$ have to be taken with a grain of salt (see the review by [127]). Recently, the anomalous ${ }^{18} \mathrm{O} /{ }^{17} \mathrm{O}$ in meteorites, $5.2 \pm 0.2$ (see the compilation in [480]), with respect to the Galactic one, $4.1 \pm 0.1$ (474]) has also been taken as a proof of the injection of material from a type II supernova exploded just before the birth of the Solar System.

\section{The calm before the storm: pre-stellar cores}

Stars like our Sun form in slowly rotating and collapsing magnetized dense cloud cores (e.g. 181]; 423]). Dense cores not associated with stars are called "starless cores" and they represent the initial conditions in the process of star formation ([399]). They are the starting point of our journey. These objects have average volume densities at least one order of magnitude larger than the surrounding medium, have typical kinetic and dust temperatures of $10 \mathrm{~K}$ and their internal energy is dominated by thermal motions (see review by [40]). Not all starless cores give birth to stars, though. Some of them reach configurations close to hydrostatic equilibrium and display kinematic features consistent with oscillations ([255]). Others show expanding motions ([408). This class of 
starless cores typically displays a relatively flat density distribution, with central densities below $10^{5} \mathrm{H}_{2}$ molecules $\mathrm{cm}^{-3}$. This is the critical density for gas cooling by gas-dust collisions ([179]) and it represents the "dividing line" for dynamical stability. Starless cores with central densities below this critical density are thermally subcritical (245) and they may disperse back into the interstellar medium. When the central densities of $\mathrm{H}_{2}$ molecules overcome $\simeq 10^{5} \mathrm{~cm}^{-3}$, starless cores become thermally supercritical and gravitational forces take over. These are the so-called pre-stellar cores, first identified by 450 in the sub-millimeter continuum and then chemically and kinematically labelled by [114] using millimeter spectroscopy. It is within pre-stellar cores that future star and planetary systems will form.

4.1 Freeze-out, deuterium fractionation and the ionization fraction

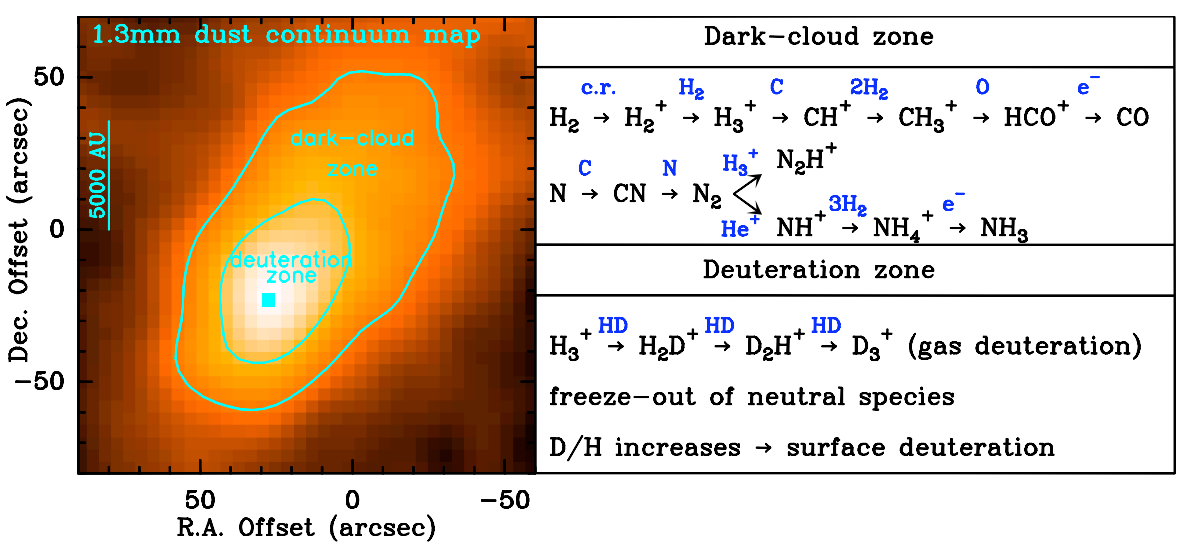

Fig. 5 The chemical zones of the prototypical pre-stellar core L1544, embedded in the Taurus Molecular Cloud Complex, at a distance of $140 \mathrm{pc}$. The background color image is the $1.3 \mathrm{~mm}$ dust continuum emission map obtained with the IRAM-30m antenna (449]). The cyan contours show the different chemical zones, with the corresponding main chemical processes listed in the right panel. Blue labels indicate reaction partners.

Pre-stellar cores span a range of number densities which goes from a few times $10^{3} \mathrm{~cm}^{-3}$ toward the outer edges, where they merge with the surrounding molecular cloud, to about $10^{7} \mathrm{~cm}^{-3}$ within the central 1,000 AU (e.g. [148; [246]), where the gas and dust temperature drops to 6-7 K ([115]; 335]). These gradients in physical properties affect the chemical structure. Fig. 5 schematically shows the main chemical processes in the two-zones of the prototypical pre-stellar core L1544, embedded in the Taurus molecular cloud. In the outer part of the core (between about 7,000 and $15,000 \mathrm{AU}$ ), the gas density is $\simeq 10^{4} \mathrm{~cm}^{-3}$ and the temperature $\simeq 10 \mathrm{~K}$. "Classical" dark-cloud chemistry is at work, with ion-molecule reactions (202]) dominating the carbon chemistry, and neutral-neutral reactions which start the transformation of nitrogen atoms 
into $\mathrm{N}_{2}$ (e.g. [205]). These reactions form the "popular" species $\mathrm{CO}, \mathrm{N}_{2} \mathrm{H}^{+}$and $\mathrm{NH}_{3}$, which are widely used to study cloud structures and kinematics.

Freeze-out. Within the central 7,000 AU, the density increases above $10^{5} \mathrm{~cm}^{-3}$, the temperature drops below $10 \mathrm{~K}$ and species heavier than He tend to disappear from the gas-phase due to the process of freeze-out (the adsorption of species onto dust grain surfaces). CO freeze-out has been measured in starless and pre-stellar cores at a 80-90\% level ([461; 82$] ; 23] ; 368])$. Nitrogen-bearing species have also been found to deplete from the gas-phase, although not as much as CO (e.g. [38; 409]; 160). The reason for this differential freezeout has to be found in the fact that N-bearing species, such as $\mathrm{N}_{2} \mathrm{H}^{+}$and $\mathrm{NH}_{3}$, experience larger production rates when neutral species (in particular $\mathrm{CO})$ start to disappear from the gas-phase. The freeze-out is a natural consequence of the quiescent nature of pre-stellar cores: once species land on a grain surfaces, they cannot thermally evaporate (because dust temperatures are $T_{\text {dust }} \leq 10 \mathrm{~K}$, typical binding energies are $E_{\mathrm{B}} \geq 1000 \mathrm{~K}$ and the thermal evaporation rate is $\left.\propto \exp \left[-E_{\mathrm{B}} /\left(k T_{\mathrm{dust}}\right)\right]\right)$ and they cannot photodesorb as interstellar photons cannot penetrate within pre-stellar cores (whose central regions have visual extinctions larger than $50 \mathrm{mag}$ ). Only a small fraction of the adsorbed species can return in the gas-phase via non-thermal desorption mechanisms mainly driven by cosmic-rays, such as dust impulsive heating due to cosmic-ray bombardment (e.g. 267) and photodesorption due to the FarUV (FUV) field produced by cosmic-ray impacts with $\mathrm{H}_{2}$ molecules (360]; [187; 396]), although molecular hydrogen formation (462] 378]) and surface reactions involving radicals (130]) may also play a role. Desorption of mantle species by FUV photons has been included in the chemical-dynamical models of L1544, to explain the recent Herschel detection of water vapor in the center of this prototypical pre-stellar core (79). Freeze-out time scales $\left(t_{\text {freeze-out }} \propto 10^{9} / n_{\mathrm{H}} \mathrm{yr}\right.$, where $n_{\mathrm{H}}$ is the total number density of hydrogen nuclei, 226]) are significantly shorter than the dynamical (free-fall) time scale $\left(t_{\text {free-fall }} \propto 4 \times 10^{7} / \sqrt{n_{\mathrm{H}}},[405]\right)$, so dust grains are expected to build thick icy mantles during the pre-stellar phase of the star formation process $(4.2)$.

Deuterium fractionation. In the cold environments of pre-stellar cores, another important process takes place: deuterium fractionation. The starting point is the exothermic reaction between $\mathrm{H}_{3}^{+}$and $\mathrm{HD}$, which produces $\mathrm{H}_{2} \mathrm{D}^{+}$ and $\mathrm{H}_{2}\left(\mathrm{H}_{3}^{+}+\mathrm{HD} \rightarrow \mathrm{H}_{2} \mathrm{D}^{+}+\mathrm{H}_{2}+230 \mathrm{~K}\right.$, 453]). This reaction cannot proceed from right to left when the kinetic temperature is below $\simeq 20 \mathrm{~K}$ and if a large fraction of $\mathrm{H}_{2}$ molecules is in para form, as expected in cold and dense cores ([153]; 337]; 424]). Therefore, the $\mathrm{H}_{2} \mathrm{D}^{+} / \mathrm{H}_{3}^{+}$abundance ratio becomes significantly larger than the $\mathrm{D}$ elemental abundance with respect to $\mathrm{H}$. When the freeze-out of neutral species (especially $\mathrm{CO}$ and $\mathrm{O}$, which are the main destruction partners of $\mathrm{H}_{2} \mathrm{D}^{+}$) becomes important, deuterium fractionation is further enhanced ([125). In fact, the deuteration zone of Fig. 5 is the region where the brightest line of ortho- $\mathrm{H}_{2} \mathrm{D}^{+}$has ever been detected (81). This deuteration "jump" allows multiply deuterated forms of $\mathrm{H}_{3}^{+}$to thrive (434]; 
[339]) and their dissociative recombinations with electrons liberate D atoms, locally increasing the $\mathrm{D} / \mathrm{H}$ ratio to values larger than 0.1 ([377]). The large $\mathrm{D} / \mathrm{H}$ ratio in the gas-phase implies efficient deuteration of surface species (in particular CO), with the consequent production of deuterated and doubly deuterated formaldehyde as well as singly, triply and doubly deuterated methanol (e.g. 421]; 101; 80; 410]; 413]). $\mathrm{HDCO}, \mathrm{D}_{2} \mathrm{CO}$ and $\mathrm{CH}_{2} \mathrm{DOH}$ have been detected in pre-stellar cores (24); 41]), while doubly and triply deuterated methanol have been detected in the envelope of young stellar objects (343; 340, see \$5).

The ionization fraction. Deuterated species are the main probes of the central regions of pre-stellar cores, the future stellar cradles. Their observation allows us to trace the kinematics (e.g. [425; [115]) and, together with the non-deuterated isotopologue, to measure the elusive electron number density $n\left(\mathrm{e}^{-}\right)$, which plays a crucial role in the dynamical evolution of the cloud. In fact, electrons and ions gyrate around magnetic field lines which permeate the clouds, and decouple from the bulk motions. During the gravitational collapse, neutral species slip through magnetic field lines and collide with molecular ions in a process called ambipolar diffusion ([304]; 399]). Depending on the fraction of ions present in the gas-phase, neutral-ion collisions can significantly slow down the collapse compared to free-fall. How do we measure the ionization degree? Using simple steady-state chemistry of (easy-to-observe) molecular ions, such as $\mathrm{HCO}^{+}$and $\mathrm{DCO}^{+}$, which form from the reaction of $\mathrm{CO}$ with $\mathrm{H}_{3}^{+}$and $\mathrm{H}_{2} \mathrm{D}^{+}$and are destroyed by electrons, it is easy to arrive at analytic expressions relating the observed $\mathrm{DCO}^{+} / \mathrm{HCO}^{+}$abundance ratio to $n\left(\mathrm{e}^{-}\right)([188$; 473 ). Using time dependent chemical codes, 83] and [39] obtained values of $x\left(\mathrm{e}^{-}\right)\left(\equiv n\left(\mathrm{e}^{-}\right) / n\left(\mathrm{H}_{2}\right)\right)$ between $10^{-8}$ and $10^{-6}$. Given that the time scale for ambipolar diffusion is $t_{\mathrm{AD}} \simeq 2.5 \times 10^{13} x\left(\mathrm{e}^{-}\right) \mathrm{yr}$ (405]), the above measurements imply values of $t_{\mathrm{AD}} \simeq 2.5 \times 10^{5}$ and $2.5 \times 10^{7} \mathrm{yr}$, factors of 2-200 larger than $t_{\text {free-fall }}$ for pre-stellar cores with an average $n_{\mathrm{H}}=10^{5} \mathrm{~cm}^{-3}$.

${ }^{15} \mathrm{~N}$-fractionation. On the one hand, no significant ${ }^{15} \mathrm{~N}$ fractionation (compared to the Solar Nebula value of $\sim 440$, see \$3.4) has been found in $\mathrm{NH}_{3}$ $\left({ }^{14} \mathrm{~N} /{ }^{15} \mathrm{~N} \simeq 350-850,[176\right.$; 334 150, [270]) toward pre-stellar cores and protostellar envelopes, and in $\mathrm{N}_{2} \mathrm{H}^{+}\left({ }^{14} \mathrm{~N} /{ }^{15} \mathrm{~N}=446 \pm 71\right.$, 44 $)$ toward the prototypical pre-stellar core L1544. On the other hand, 299] and Hily-Blant et al. (submitted) found significant ${ }^{15} \mathrm{~N}$-enrichment in $\mathrm{HCN}$ toward pre-stellar cores (between 70 and 380). Similar values have been found by [3] in HNC observations of star-forming regions across the Galaxy. It is interesting to point out here that the ${ }^{15} \mathrm{~N}$-fractionation observed in comets $(3.4$ has been measured for $\mathrm{CN}$ and $\mathrm{HCN}\left({ }^{14} \mathrm{~N} /{ }^{15} \mathrm{~N} \sim 130-170\right.$, [50]). This differential ${ }^{15} \mathrm{~N}$ fractionation for amines and nitriles has been recently reproduced in chemical models of dense clouds by [466], who suggest that the processes able to reproduce the observed differentiation could be at the origin of the poor correlation between D- and ${ }^{15} \mathrm{~N}$-fractionation observed in some primitive material in our Solar System (3.4). Thus, a further link between pre-stellar core chemistry and the Solar System composition has been found (see \$7). 
4.2 Ice formation and evolution

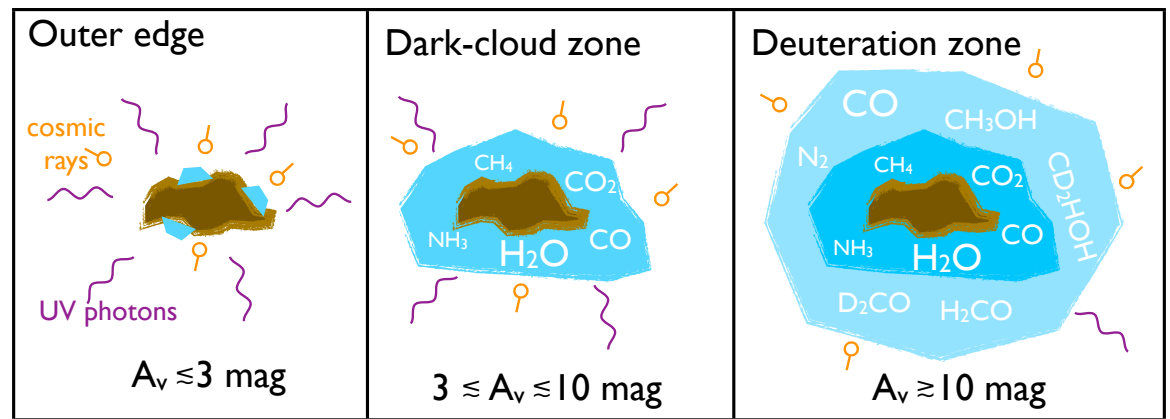

Fig. 6 Ice mantle evolution within a pre-stellar core, from the outer-edge, where the core merges with the surrounding molecular cloud, to the dark-cloud zone and deuteration zone as depicted in Fig.5 Ice mantles become thicker and richer in complex organic molecules moving toward the center of a pre-stellar core, where star- and planet-formation takes place.

Interstellar dust grains are crucial for the chemical and physical evolution of interstellar clouds and for our astrochemical origins. First of all, hydrogen atoms can quickly scan their surfaces, meet and form volatile $\mathrm{H}_{2}$ molecules at rates large enough to defeat $\mathrm{H}_{2}$ photodissociation due to the interstellar radiation field (212] 351]; 88]; 119]). Thus, dust grains are responsible for the transition of interstellar gas in our Galaxy (as well as in external galaxies) from atomic to molecular - the first step toward chemical complexity. Secondly, they are efficient absorbers of the FUV photons, so that they act as "UV-filters", protecting molecules within clouds from the UV destructive action. Thirdly, they catalyze the formation of important species, in particular $\mathrm{H}_{2} \mathrm{O}$, with such high efficiency that more than $30 \%$ of oxygen atoms are locked into water ice as soon as the visual extinction reaches values $\geq 3$ mag (e.g. [310; 210]; 455; [104]). Finally, they become the main gas coolants in the central regions of pre-stellar cores, where the densities are above $\simeq 10^{5} \mathrm{~cm}^{-3}$, the temperatures fall below $10 \mathrm{~K}$ and species heavier than He (including important coolants such as $\mathrm{CO}$ ) are mostly frozen onto their surfaces. In such conditions, the freeze-out rate will become even more extreme and dust grains should develop thick ice mantles. How thick? A simple estimate can be made considering that levels of CO freeze-out of about $90 \%$ are seen within the central pre-stellar core regions (see \$4.1). Assuming that all species heavier than Helium are affected by a similar amount of freeze-out (including nitrogen; 205]), then in clouds with total hydrogen density of $2 \times 10^{6} \mathrm{~cm}^{-3}$, the total number density of heavy species frozen onto dust grains is about $1.3 \times 10^{3} \mathrm{~cm}^{-3}$. Further assuming that they are combined in molecules with two heavy elements on average (e.g. $\mathrm{CO}, \mathrm{CH}_{3} \mathrm{OH}, \mathrm{CO}_{2}, \mathrm{H}_{2} \mathrm{O}$ ), the total number of solid species will be about $660 \mathrm{~cm}^{-3}$. Now, we just need to divide this number by the total number of sites on an average grain with radius $\left.0.1 \mu \mathrm{m}\left(\simeq 10^{6} ; 196\right]\right)$ to have the number 
of monolayers $(\simeq 250)$. Considering a monolayer thickness of about $1 \AA$, the total mantle thickness is then $2.5 \times 10^{-6} \mathrm{~cm}$, or about a quarter of the grain radius. Such thick mantles boost dust coagulation ([331]).

What are the main chemical processes on the surface of dust grains? Our understanding is based on (i) observations of absorption features along the line of sight of stars located behind molecular clouds or protostars embedded in dense cores (e.g. 456] and references therein), and on (ii) laboratory work (e.g. 4452; 208]; 300; 219; [161; [136]). From these studies, we now know that surface reactions are mainly association reaction: oxygen is transformed into water via successive association reactions with hydrogen (e.g. $\mathrm{O}+\mathrm{H} \rightarrow$ $\mathrm{OH} ; \mathrm{OH}+\mathrm{H} \rightarrow \mathrm{H}_{2} \mathrm{O}$, but see $\$ 4.2 .1$ for more pathways to water ice); similarly, $\mathrm{CO}$ is transformed first into formaldehyde, $\mathrm{H}_{2} \mathrm{CO}$, and then into methanol, $\mathrm{CH}_{3} \mathrm{OH}$, via two and four association reactions, respectively; atomic nitrogen saturates into ammonia, $\mathrm{NH}_{3}$. Other important processes are photoprocesses and cosmic-ray bombardments. Photoprocesses are experimentally found to promote the formation of organic species more complex than $\mathrm{CH}_{3} \mathrm{OH}([175]$; 34; 320; 327) up to amino acids (e.g. 42; 307; 306]) and allow solid species to return into the gas-phase ([326]; 321]). Cosmic-rays, unlike UV photons, traverse dense cores relatively unhampered, although their flux may be reduced by a factor of a few by the mirroring effect of magnetic fields (334]). When colliding with dust grains, they can alter mantle compositions (e.g. 338; 220; 301; 400; 53]; 349]). Cosmic rays also play a crucial role in molecular desorption, as mentioned in the previous section. Surface chemistry is one of the most challenging disciplines in astrochemistry, but in the recent years several models have been successful in reproducing the observed abundance of some simple and complex species (e.g. [7]; 170]; 210; [120; 86]; 411]; 410]).

The picture that has emerged from the combination of observations, laboratory work and modeling is sketched in Fig 6, which shows the evolution of a dust grain mantle from the outer edge to the central regions of a pre-stellar core embedded in a molecular cloud bathed by the interstellar radiation field (with reference to Fig 5 to locate the various zones). At the outer edge of the pre-stellar core, photoprocesses are important and the ice mantles are just beginning to form. Here, oxygen atoms are transformed into water, carbon (still not locked in $\mathrm{CO}$ ) into methane $\left(\mathrm{CH}_{4}\right)$ and nitrogen into ammonia. Water dominates the mantle composition (probably reflecting the larger cosmic abundance of oxygen relative to $\mathrm{C}$ and $\mathrm{N}$ ). Moving toward the dark-cloud zone (where the pre-stellar core merges with the molecular cloud within which it is embedded), UV photons are absorbed by dust grains, CO becomes the second most abundance molecule (after $\mathrm{H}_{2}$ ) and the mantle starts to accumulate $\mathrm{CO} . \mathrm{CO}_{2}$ also starts to form, either via cosmic-ray bombardment ([220]) and/or via the $\mathrm{CO}+\mathrm{OH}$ reaction ([319]; 221]; [317]; 169]). Here, the limited amount of $\mathrm{CO}$ freeze-out limits the degree of deuteration to levels of $\leq$ a few $\%$ (as measured from the observed $\mathrm{DCO}^{+} / \mathrm{HCO}^{+}$abundance ratio; e.g. 84]). Deeper into the pre-stellar core, CO molecules are mostly in solid form, deuteration processes are dominant and the $\mathrm{D} / \mathrm{H}$ ratio reaches values above 0.1 (see $\$ 4.1$. When freeze-out is dominant, the main reactive species landing on dust 
grain surfaces are atomic $\mathrm{H}$ and $\mathrm{D}$. Thus, $\mathrm{CO}$ is not only hydrogenated into formaldehyde and methanol, but also deuterated. Large amounts of deuterated and multiply deuterated $\mathrm{H}_{2} \mathrm{CO}$ and $\mathrm{CH}_{3} \mathrm{OH}$ are produced (see 4.1 .

\subsubsection{The origin of water}

Extra attention is given here to the production of water, because of its dominant presence in interstellar ices and its crucial role in our astrochemical origins. Recent measurements of water vapor toward a pre-stellar core with the Herschel Space Observatory and the use of chemical/dynamical/radiative transfer models, allowed [79] to measure a total mass of water vapor of 0.5 Earth masses within the central 10,000 AU and predicted about 2.6 Jupiter masses of water ice (thus, plenty of ice to boost dust coagulation and the formation of giant planets via core accretion models, e.g. 357). From observations of water ices in molecular clouds (e.g. [456] and references therein), it is now well established that water ice forms on the surface of dust grains in regions of molecular clouds where the visual extinction is at least 3 mag (when the impinging radiation field is close to the average Galactic value, called the Habing field; larger extinctions are needed for stronger fields). For lower extinction values, the interstellar UV field does not allow dust grain surfaces to accumulate a significant amount of water molecules, as they are efficiently photodesorbed (321). Laboratory work shows that $\mathrm{H}_{2} \mathrm{O}$ can form via hydrogenation of atomic oxygen ([207; ; 136]; 225]), molecular oxygen (219]; 300]), ozone (302]; 381]) and via $\mathrm{OH}+\mathrm{H}_{2}$ at $10 \mathrm{~K}(318)$. As the abundance of water ice in molecular clouds, within which pre-stellar cores form, is already large (close to $10^{-4}$ w.r.t. $\mathrm{H}_{2}$ molecules; e.g. [457]), we now generally believe that the main production of water happens before the formation of a pre-stellar core, as also found by chemical models (e.g. 7]; 210; ; 87; 411]). This suggests that also the production of heavy water must be regulated by the molecular cloud characteristics. This is an important point, as the $\mathrm{HDO} / \mathrm{H}_{2} \mathrm{O}$ ratio is well measured on Earth, comets and asteroids $(\$ 3.1$, as well as in star-forming regions (\$5.4). Therefore, one could use our current understanding of surface chemistry and the observed $\mathrm{HDO} / \mathrm{H}_{2} \mathrm{O}$ abundance ratios in star-forming regions to find the link between interstellar chemistry and the Solar System.

Cazaux et al. (86]) predict that significant variations in the $\mathrm{HDO} / \mathrm{H}_{2} \mathrm{O}$ ratio can be attributed to small variations of the dust temperature at the time of ice formation. In particular, if the dust temperature is lower than $\simeq 15 \mathrm{~K}$, the $\mathrm{HDO} / \mathrm{H}_{2} \mathrm{O}$ ratio is predicted to be $\leq 0.01 \%$, because, in these conditions, a large fraction of the dust surface is covered by $\mathrm{H}_{2}$ molecules, allowing the reaction of $\mathrm{H}_{2}+\mathrm{O}$ to proceed despite the large barrier of $3000 \mathrm{~K}$ (318 did not find evidence in the laboratory that this reaction is indeed proceeding, but more laboratory work is ongoing to assess this). The $\mathrm{HDO} / \mathrm{H}_{2} \mathrm{O}$ ratio in these conditions simply reflects the $\mathrm{HD} / \mathrm{H}_{2}$ ratio, always close to the interstellar $\mathrm{D} / \mathrm{H}$ value $\left(\simeq 1.5 \times 10^{-5},[329)\right.$. For dust temperatures above $\simeq 15 \mathrm{~K}, \mathrm{H}_{2}$ molecules do not stay on the dust surface for long (as their evaporation rate becomes an increasingly large fraction of their accretion rate) and water formation will 
mostly happen via the reaction of oxygen with atomic hydrogen. As the gasphase $\mathrm{D} / \mathrm{H}$ ratio sharply increases above the cosmic deuterium abundance when ice formation takes place (see Figure 1 of [86]), then the $\mathrm{HDO} / \mathrm{H}_{2} \mathrm{O}$ ratio can be as large as a few \%. In this scenario, our Solar System formed in a pre-stellar core embedded in a molecular cloud with dust temperature slightly above $15 \mathrm{~K}$. Taquet et al. (411), using a multilayered formation mechanism of ice mantles (411]), find that water is formed first on dust surfaces and that the $\mathrm{HDO} / \mathrm{H}_{2} \mathrm{O}$ ratio depends on the (poorly constrained) ortho:para ratio of $\mathrm{H}_{2}$, on the cloud volume density and, to a lesser extent, on the dust temperature and visual extinction. However, water deuteration can also occur in the gas-phase: Thi et al. (419]) found that significant deuteration levels $\left([\mathrm{HDO}] /\left[\mathrm{H}_{2} \mathrm{O}\right] \simeq 10^{-3}-10^{-2}\right)$ can be produced without surface reactions and at high temperature $(T>100 \mathrm{~K})$, in the inner regions of protoplanetary disks (\$6.2). The fractionation occurs because of the difference in activation energy between deuteration enrichment and the back reactions.

\subsection{Complex organic molecules}

In the freezing cold of dark clouds and pre-stellar cores, active gas-phase and surface chemistry produce complex organic molecules (COMs). Since the '80s, organic molecules have been discovered in the TMC-1 dark cloud, part of the Taurus Molecular Cloud complex: methyl cyanide $\left(\mathrm{CH}_{3} \mathrm{CN} ;\right.$ 290]), methylcyanoacetylene $\left(\mathrm{CH}_{3} \mathrm{C}_{3} \mathrm{~N}\right.$, 67]), acetaldehyde $\left(\mathrm{CH}_{3} \mathrm{CHO}\right.$; 289]), ketene $\left(\mathrm{CH}_{2} \mathrm{CO}\right.$; 222] $)$, methanol $\left(\mathrm{CH}_{3} \mathrm{OH}\right.$; 159]), methylcyanodiacetylene $\left(\mathrm{CH}_{3} \mathrm{C}_{5} \mathrm{~N}\right.$, [404]), methyltriacetylene $\left(\mathrm{CH}_{3} \mathrm{C}_{6} \mathrm{H}\right.$, [369]), propylene $\left(\mathrm{CH}_{2} \mathrm{CHCH}_{3}\right.$, 281]), methyldiacetylene $\left(\mathrm{CH}_{3} \mathrm{C}_{4} \mathrm{H}\right)$, cyanopolyynes $\left(\mathrm{HC}_{2 \mathrm{n}+1} \mathrm{~N}, n=0,1, \ldots, 5\right)$ and $\mathrm{C}_{2 \mathrm{n}+1} \mathrm{~N}$ radicals (447]; 206]; 328]; 236]) and the negative ions $\mathrm{C}_{6} \mathrm{H}^{-}, \mathrm{C}_{8} \mathrm{H}^{-}$(291]; 72]). Complex organics have also been found in two pre-stellar cores: L183 $\left(\mathrm{CH}_{3} \mathrm{CHO}\right.$; 289]; $\mathrm{HCOOH}$, 372]) and L1689B $\left(\mathrm{CH}_{3} \mathrm{CHO}, \mathrm{HCOOCH}_{3}, \mathrm{CH}_{3} \mathrm{OCHO}\right.$, $\left.\mathrm{CH}_{2} \mathrm{CO},[25]\right)$. The chemistry of C-bearing species such as cyanopolyynes and $\mathrm{CH}_{3} \mathrm{C}_{5} \mathrm{~N}$ can be understood if the gas-phase is carbon-rich $\left.(\mathrm{C} / \mathrm{O} \simeq 1.2 ; 446]\right)$ or if polycyclic aromatic hydrocarbons (PAHs) are included in the chemistry (with a standard $\mathrm{C} / \mathrm{O}$ abundance ratio of $\simeq 0.4$, [44]). More problematic is the explanation of complex O-bearing species, such as methanol, which require surface chemistry. Garrod et al. ([171]) assumed that the energy released during the formation process could be at least partially used for the surface species to desorb upon formation, reconciling observations with theory for $\mathrm{CH}_{3} \mathrm{OH}$ and propylene (if the desorption of this species is efficient). Oxygen-bearing species more complex than methanol can also be formed on the surface of low temperature dust grains if a source of UV photons is present $(4.2)$. For example, in the laboratory experiments of [320], it has been shown that the photodissociation of $\mathrm{CH}_{3} \mathrm{OH}$ produces radicals such as $\mathrm{CH}_{3}$ and $\mathrm{CH}_{3} \mathrm{O}$ (recently discovered in a dark cloud by Cernicharo et al., in press), which can then recombine to form $\mathrm{CH}_{3} \mathrm{OCH}_{3}$ or react with $\mathrm{CHO}$ (probably produced by the photodissociation of solid $\mathrm{CH}_{4}$ and $\mathrm{H}_{2} \mathrm{O}$, see below) to form $\mathrm{CH}_{3} \mathrm{CHO}$ and 
$\mathrm{HCOOCH}_{3}$, respectively. Interstellar UV photons are expected to be important up to values of visual extinction of $\simeq 3 \mathrm{mag}$ (e.g. [210]), where CO is not yet significantly frozen onto dust grains (see Fig. 5). Deeper into pre-stellar cores, a significantly more tenuous field of UV photons can be produced by the collisions of cosmic-rays with $\mathrm{H}_{2}$ molecules ([360]; [187]). It is not yet clear if this cosmic-ray induced field is able (i) to produce enough radicals, (ii) to furnish them enough energy to move on the surface, recombine and form complex molecules, and (iii) to release them into the gas-phase where they are observed (see also the discussion in 411). Consequently, it is not yet clear whether models are able to reproduce the abundances of complex molecules observed by 25$]$.

In summary, possible first steps toward the formation of COMs in the ice (before the switch-on of the protostar) are:

(1) Production and storage of radicals. In the molecular cloud within which the pre-stellar core forms, at $A_{\mathrm{V}} \simeq 3 \mathrm{mag}$, interstellar UV photons can still partially dissociate important ice components $\left(\mathrm{H}_{2} \mathrm{O}\right.$ and $\left.\mathrm{CH}_{4}\right)$ and some of the products can be trapped within the ice, which already contains significant fractions of water (e.g. 104). Alternatively, because of the multilayered nature of icy mantles, radicals can be stored in the inner layers during mantle formation ([41] ).

(2) Radical-radical reactions. As the density increases and CO starts to freezeout onto the first water-dominated ice layers, the $\mathrm{CO}$ is transformed into $\mathrm{CH}_{3} \mathrm{OH}$ more and more with increasing freeze-out (given that with the freezeout of $\mathrm{CO}$ and $\mathrm{O}$, the $\mathrm{H} / \mathrm{O}$ and $\mathrm{H} / \mathrm{CO}$ abundance ratios in the gas-phase increase, as the number density of $\mathrm{H}$ atoms is kept about constant to $1 \mathrm{~cm}^{-3}$ by the cosmic-ray dissociation and surface re-formation of $\mathrm{H}_{2}$ molecules). The energy released during the formation of methanol is partially used by methanol itself to evaporate and partially released as heat on the icy surface, allowing some of the previously trapped radicals to move. The new radicals produced in the dissociation of $\mathrm{CH}_{3} \mathrm{OH}$ by cosmic-ray-induced UV photons (and probably some of the intermediate compounds produced during the $\mathrm{CO} \rightarrow \mathrm{CH}_{3} \mathrm{OH}$ conversion) will then participate in the formation of the observed complex organic molecules (e.g. 320]). As for the case of methanol, the energy released in the process of formation of these COMs can be partially used to return in the gas-phase. The impulsive heating of dust grains due to the impact of heavy cosmic rays (267]) may also temporarily enhance the mobility of the stored radicals, allowing complex molecule formation.

We emphasize that the above steps remain highly speculative and more experimental and theoretical work is necessary to better understand the grain surface chemistry processes. Given that the observed COMs are building blocks of biologically important species, this once again underlines the importance of pre-stellar cores for the first steps toward our astrochemical origins. 


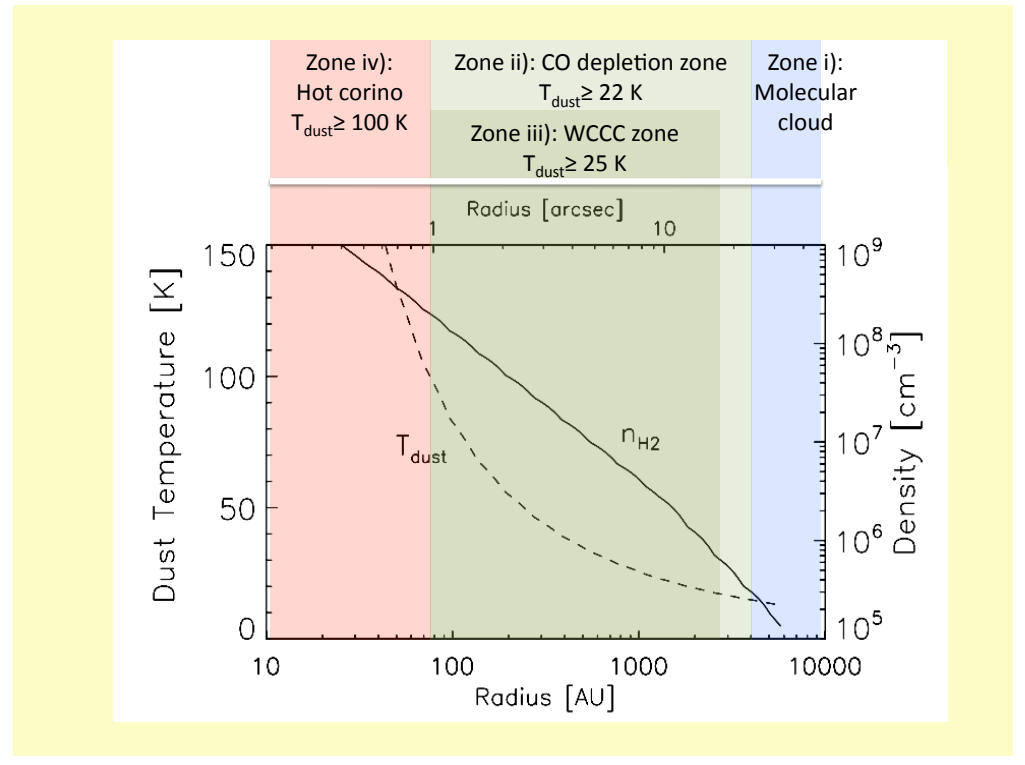

Fig. 7 Temperature and density profile of IRAS16293-2422, the prototype for chemical studies in Class 0 sources (from [117]). The colored boxes represent the four chemical zones described in the text: i) molecular cloud, ii) CO depletion, iii) Warm-Carbon-ChainChemistry (WCCC); iv) hot corino.

\section{The cocoon phase: protostars}

Once the collapse starts, the gravitational energy released at the center of the infalling envelope is converted into radiation. During the first phases of star formation, this is the main source of the protostar luminosity $L_{*}$ and it is given by $L_{*}=G M_{*} \dot{M} / R_{*}$, where $M_{*}$ and $R_{*}$ are the mass and radius of the central object, and $\dot{M}$ is the mass accretion rate. The approximate structure of the envelope, as derived by observations of the continuum and line emission (e.g. 91; 231; 379]) is reported in Fig. 7. Both the density and temperature increase toward the center. Similarly, the velocity of the infalling gas increases with decreasing distance from the center with an $r^{-1 / 2}$ power law, although part of the envelope may not be collapsing yet. The infall motion has proved difficult to disentangle from the outflow motions, but high spatial and spectral resolution observations recently obtained with ALMA $^{3}$ have succeeded to probe it unmistakably toward IRAS16293-2422 (350]). Finally, new Herschel observations provide a much more complicated picture where, at least in some sources, the cavity created by the outflowing gas is illuminated and heated by the UV photons of the central star, making the interpretation of the observed lines not straightforward (440]).

3 The Atacama Large Millimeter/submillimeter Array. 
5.1 The chemical composition of protostellar envelopes: a powerful tool to understand the present and the past

Chemistry has been recognised to be a powerful diagnostic tool in several fields of astrophysics to understand the present and the past of the studied object. For example, at large scale, the chemical enrichment in stars throughout the Milky Way tells us about different star populations and ages, and, consequently, how the Milky Way formed (e.g. [186]). Similarly, at much smaller scales, the chemical composition in protostellar envelopse tell us about their present status and past history.

Figure 7] shows the approximate and very simplified density and temperature profiles of a typical protostellar envelope. To a scale of $\geq 100 \mathrm{AU}$, a roughly spherical envelope heated by the internal new born star this is probably a correct description. However, at smaller scales, the envelope is not spherical, because of the presence of a circumstellar disk (\$6) and the presence of multiple sources, as in the case of IRAS16293-2422 and NGC1333-IRAS4 (e.g. 472]), among the two most studied examples of solar-type protostars. Nonetheless, from a chemical point of view, four major zones can be identified (Fig. 7): i) an outer zone, with the same chemical composition as that of the placental molecular cloud; ii) a CO depleted zone, usually called cold envelope, with the chemistry is very similar to that of pre-stellar cores (\$4); iii) a $\mathrm{CH}_{4}$ ice sublimation region, where the chemistry is dominated by the warm carbon chain chemistry, called WCCC, triggered by sublimation of the methane from the grain mantles; iv) the hot corino zone, where the chemistry is dominated by the water-matrix grain mantle sublimation and hot gas chemistry. The transition between zones ii) to iv) is determined by the dust temperature, which governs the sublimation of the icy mantles, whereas the $\mathrm{CO}$ depleted region depends on the density and age of the protostellar envelope. In the following we summarise the characteristics of the four zones.

Zone i) The chemical composition in this zone is similar to typical molecular clouds, with no particularly important freeze-out of species. Whether this zone is present or not in a protostellar envelope depends on the envelope density and age, which determines the existence of zone ii).

Zone ii) As described in $\$ 4$, if the density and age of the envelope are high enough, molecules freeze-out onto dust surfaces. Important for the various reasons again described in 8 is the region where $\mathrm{CO}$ freezes out, defined by a dust temperature lower than about $22 \mathrm{~K}$. Jørgensen et al. (232]) found that a large fraction of Class 0 and Class I protostars have CO-depleted regions in their envelopes, typically where the density is larger than $\sim 10^{5}$ $\mathrm{cm}^{-3}$. Models of the chemistry in young protostellar envelopes provide a theoretical interpretation to these observations (e.g. [261]).

Zone iii) When the dust temperature exceeds the methane sublimation temperature, $\sim 25 \mathrm{~K}$, the chemistry is governed by the injection of methane in the gas-phase, if the $\mathrm{CH}_{4}$ abundance is larger than $\sim 10^{-7}$. In this case, $\mathrm{CH}_{4}$ becomes a major destruction partner for $\mathrm{C}^{+}$, starting the efficient 
formation of C-chain molecules in the relatively warm (30-60 K) gas ( $[7$; [198; [197]). So far, only a few protostellar envelopes with very abundant C-chain molecules have been discovered. L1527 is the prototype of this class of sources, called Warm-Carbon-Chain-Chemistry (WCCC) sources (383; [385]; 385]). Note that the abundance of methane has been indirectly inferred in those sources by modelling the observed C-chain molecules, as gaseous $\mathrm{CH}_{4}$ does not have observable rotational transitions.

Zone iv) When the dust temperature exceeds about $100 \mathrm{~K}$, the grain mantles evaporate and all species trapped in them are released in the gas-phase, giving rise to a rich chemistry, first discovered in high-mass protostellar envelopes and called hot core chemistry (e.g. [48), and successively unveiled in lowmass protostellar envelopes ([89]). However, as it will be discussed in detail in $\$ 5.2$, the chemical composition of low- and high- mass cores is not identical.

The transition zones in Fig. reffig:i16293-structure are, of course, approximate, as laboratory experiments show that ice sublimation is a complex process where molecules are released into the gas through several steps at different dust temperatures (e.g. 442]). Also, the outflows emanating from the central objects open up cavities which are directly illuminated by the UV photons of the new born star (e.g. 429]; 479;; 440]). In these cases, large PhotonDominated-Regions (PDRs) may dominate and mask the molecular emission from the various zones, depending on the extent of the cavity.

As already mentioned, the presence of the WCCC zone (zone iii) depends on the abundance of methane in the dust mantles. Methane is formed, as the vast majority of the grain mantles, during the pre-stellar phase (\$4). Specifically, it is believed to form by hydrogenation of neutral carbon. However, in typical molecular clouds, neutral carbon is a rare species because of the efficient formation of $\mathrm{CO}$. Therefore, to have a large quantity of iced $\mathrm{CH}_{4}$, one needs particular conditions, namely a relatively high abundance of neutral carbon in the gas-phase. This occurs when the transition from the diffuse cloud to molecular cloud is very fast, and a substantial fraction of carbon atoms freezeout into the grain mantles before the CO formation is achieved (e.g. 197]). Therefore, the presence of a WCCC zone may be a signature of fast collapse (383]), for example triggered by a shock from a nearby forming star or two encountering diffuse clouds. Alternatively, if the pre-stellar core is embedded in a relatively tenuous cloud, $\mathrm{CO}$ photodissociation could still play a role and led to a large amount of methane ice. Unfortunately, the limited number of observations do not allow us to go much further in the interpretation of this peculiar chemistry, and more studies are needed to fully exploit it. In the same vein, the chemical composition in the hot corino zone, as well as the observed molecular deuteration, are all largely influenced by the pre-stellar phase. These cases will be discussed in detail in the following paragraphs.

Last, a potentially powerful diagnostic is provided by the relative abundances of isomers of the same generic formula. Since the interstellar chemistry is dominated by kinetics, different isomers have in principle the imprint of the 
different chemical formation routes. Therefore, the isomer relative abundances help understanding the reactions at work and, consequently, how well we understand the interstellar chemistry. A puzzling and interesting example is provided by the isocyanic acid (HNCO) and its isomers fulminic acid (HCNO) and cyanic acid (HOCN), which have zero energies respectively 71 and $25 \mathrm{kcal} / \mathrm{mol}$ above $\mathrm{HNCO}$. In cold gas, the $\mathrm{HNCO} / \mathrm{HCNO}$ and $\mathrm{HNCO} / \mathrm{HOCN}$ abundance ratios are about 50 , whereas in warm gas $\mathrm{HNCO} / \mathrm{HCNO}$ is about 50 and $\mathrm{HNCO} / \mathrm{HOCN}$ more than 5 times larger (280]). The difference of abundances between the different isomers is thought to be due to the different chemical routes of formation and destructions (364]). However, the available gas-phase and gas-grain+gas-phase models have some difficulties in reproducing the observations and the results very much depend on the assumption made on the $\mathrm{CH}_{2}+\mathrm{NO}$ reaction rate coefficient. Even more puzzling, these models do not explain the observed difference in the $\mathrm{HCNO} / \mathrm{HOCN}$ ratio between cold and warm sources. Marcelino et al. ([280]) speculate the presence of a mechanism that converts HCNO into HOCN, despite the large energy barrier necessary for the isomerisation. On the other hand, Lattelais et al. (257]) already noted that a pseudo-isomerisation seems to occur to the majority of species where different isomers have been detected. They studied 14 species and 32 isomers and found that the larger the energy difference, the larger the abundance ratio between the most stable species and its isomer, with a few exceptions. They called it the "minimum energy principle" and its origin is still unclear, as the isomerisation barriers are generally very large and different isomers are formed from different "mother" species.

Similar arguments on the diagnostic value applies for the isotopologues of a species. Nice examples are provided by the CCS and CCH studies by Sakai and collaborators (e.g. [382]; 384]). Studying the abundance ratio of ${ }^{13} \mathrm{CCS} / \mathrm{C}^{13} \mathrm{CS}$ and ${ }^{13} \mathrm{CCH} / \mathrm{C}^{13} \mathrm{CH}$, they constrained the formation routes of CCS and $\mathrm{CCH}$ and demonstrated that the ${ }^{12} \mathrm{C} /{ }^{13} \mathrm{C}$ depends on the position of the carbon in the chain.

\subsection{The chemical complexity in hot corinos}

In the 90s, several abundant complex organic molecules (COMs) were discovered in an unbiased spectral survey of the prototype massive star forming region, the Orion Molecular Cloud (48]). Soon after, a similar rich chemistry was observed in several other massive protostellar envelopes. The proprieties of the line emission indicate that these COMs reside in compact $(\leq 0.01 \mathrm{pc})$, dense $\left(\geq 10^{7} \mathrm{~cm}^{-3}\right)$ and hot $(\geq 100 \mathrm{~K})$ regions, soon called "hot cores". A simple and obvious interpretation is that the observed rich chemistry is due to the sublimation of some species from the grain mantles, called "mother" or "primary" species, and the synthesis of others, called "daughter" or "secondary" species, thanks to the high gas temperature (e.g. [100]). Almost two decades later, similar results were obtained toward the envelope of the prototype low-mass protostar IRAS16293-2422 ([96] [89]), where several COMs 
were detected. Since then, more low-mass hot cores have been discovered and, to distinguish them from the high-mass hot cores, they were called hot corinos (60; 61; 62] 256]; 230; see also the review by 203]). Hot corinos differ from hot cores not only for the smaller sizes, lower temperatures and densities, but also chemically. In fact, when normalized to methanol or formaldehyde, hot corinos have typically one order of magnitude more abundant COMs (such as $\mathrm{HCOOCH}_{3}$ or $\mathrm{CH}_{3} \mathrm{OCH}_{3}$ ) than hot cores ( 90 ; [62; 203]; 325]; 111]). The difference in the richness and COMs abundances between hot cores and hot corinos is likely due to various factors. Among them, two certainly play a major role: i) the gas temperature, which governs the neutral-neutral reactions that often possess large activation energy barriers; ii) the composition of the sublimated ices, governed by the past pre-stellar history (4).

In addition to being weak line emitters and small objects, the study of hot corinos is also complicated by the fact that low-mass protostars are often binary or multiple systems (as in the case of high-mass protostars). The hot corino prototype IRAS16293-2422 is in fact a binary system and the two objects composing it, called $\mathrm{A}$ and $\mathrm{B}$ in the literature, show definitively a different chemistry (see for example the recent articles by [85] and [229], and reference therein). To illustrate this aspect, Fig. 8 shows a sketch of the chemical composition of IRAS16293-2422, based on the analysis of the single-dish unbiased spectral millimeter and sub-millimeter survey carried out by [85] and confirmed by the submillimeter interferometric unbiased survey of [229]. Four groups of species are identified:

Group I: Millimeter lines from simple molecules, like $\mathrm{CN}$ and $\mathrm{HCO}^{+}$, are dominated by the cold envelope. Also, emission from simple carbon-chains are associated with the cold envelope (see the discussion on their chemistry in \$5.1). Group II: Source A is rich in N- and S- bearing molecules.

Group III: Source B is rich in O-bearing COMs.

Group IV: Molecules like $\mathrm{CH}_{3} \mathrm{OH}, \mathrm{H}_{2} \mathrm{CO}, \mathrm{CH}_{3} \mathrm{CCH}$ and OCS emit low-lying lines in the cold envelope and high-lying lines in the two sources A and B.

The obvious question is: why are source A and B so chemically different? They must have had a similar composition of the sublimated ices, as they belong to the same core, so that the difference is probably originating from the different evolutionary status caused by the difference in mass of the two objects ([61]; 85]; 350]; 230]). However, so far no attempt has appeared in the literature to theoretically model the two sources to understand what exactly causes the observed chemical differences.

Finally, as mentioned in 4 . COMs are predicted to be formed on grain surfaces. Four fundamental steps are involved: i) freeze-out of atoms and simple molecules (such as $\mathrm{O}$ and $\mathrm{CO}$ ) on the grain surface; ii) successive additions of $\mathrm{H}$ atoms to form hydrogenated species (such as $\mathrm{CH}_{3} \mathrm{OH}$ ); iii) formation and trapping of radicals, such as $\mathrm{CH}_{3}$, on the grain surfaces; iv) combination of radicals to form COMs in the warm-up period. While laboratory experiments and quantum chemistry calculations have tested and quantified the second step, the third step is still a matter of debate. Garrod \& Herbst [168] and subsequent works from the same authors assume that the radicals are formed 


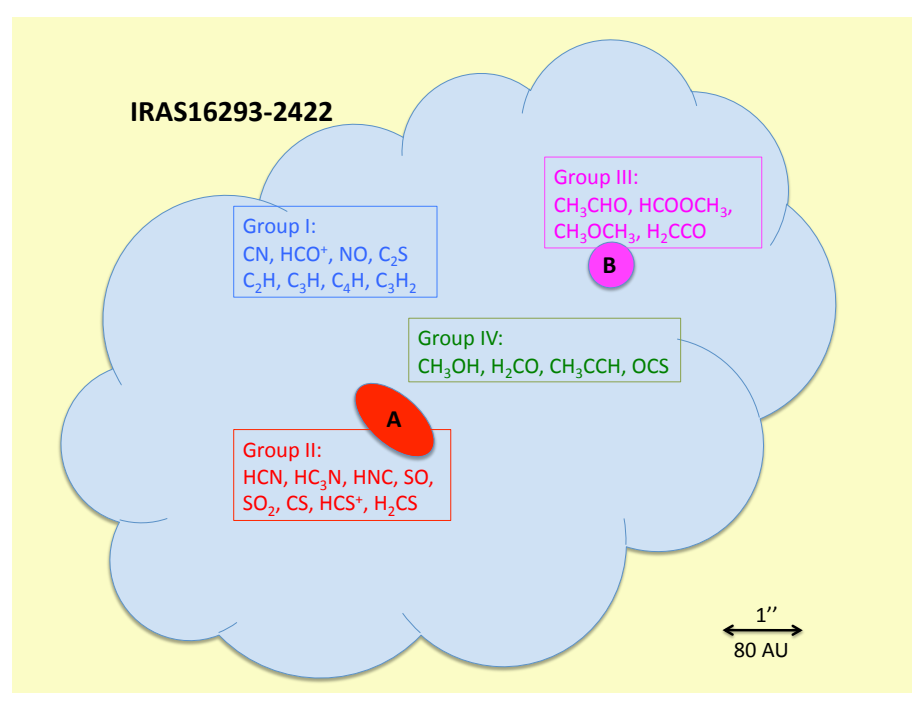

Fig. 8 Sketch of the chemical composition of the protostellar envelope of IRAS16293-2422, a protobinary system composed of two sources, A and B, as marked. The four boxes list the species in the different components of the system: species in Group I are associated with the cold envelope surrounding A and B; species in Group II are associated with source A and in Group III with source B; species in Group IV are present in the cold envelope and the two sources.

from the secondary UV photons emitted by the interaction of cosmic rays with $\mathrm{H}_{2}$ molecules. Specifically, it is assumed that UV photons break iced species like $\mathrm{CH}_{3} \mathrm{OH}$ into radicals like $\mathrm{CH}_{3}$ and that the broken pieces remain frozen on the grains, which may not be necessarily the case. On the other hand, Taquet et al. [411] showed that radicals can indeed be trapped in the grain mantles without the intervention of UV photons, just because of the intrinsic layered structure of the forming mantle.

However, it is important to emphasize that, whatever is the possible origin of the radicals, models still fail to reproduce the observed amount of COMs. For example, Fig. 9 shows the comparison between the observed and predicted methyl formate abundance normalized to the methanol one. Published models are off by at least one order of magnitude. Considering that COMs are also observed in pre-stellar cores (see $\$ 4$ and outflows $(\$ 5.3$, something basic on how COMs are formed in the ISM must still escape our understanding.

\subsection{The chemical complexity in molecular outflows}

The birth of a star is accompanied by a violent and substantial ejection of material simultaneous to the accretion toward the central object. The process has an enormous importance in the star formation process because i) it allows 


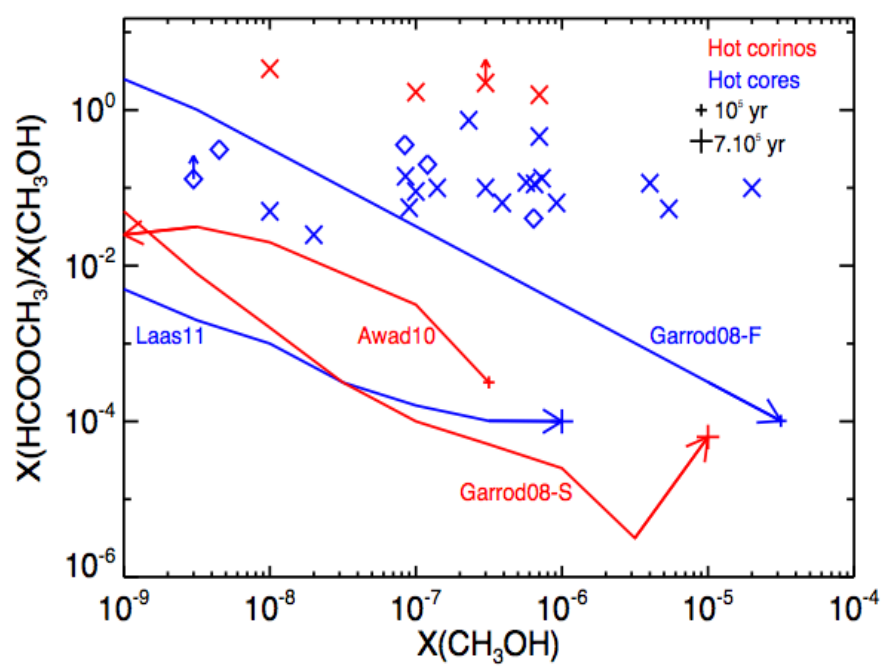

Fig. 9 Observed (red and blue crosses: hot corinos and cores) and predicted (continuum lines) methyl formate normalized to methanol abundance as function of methanol abundance. The blue lines refer to models of hot cores (172] and 254]) and red lines to models of hot corinos ([172] and [21]). Figure from [411, with permission. It is probably safe to assume that the plotted values are correct within one order of magnitude.

the infalling matter to lose angular momentum and accrete onto the central object, and ii) the ejected material interacts with the surroundings, deeply modifying it and completely destroying, in some cases, the parental cloud (e.g. [265]; 397]; 15]; Lopez-Sepulcre et al., submitted). The ejected material creates shocks at the interface between the outflowing jet and the quiescent material. Those shocks are chemically rich sites, showing a chemical composition very similar to hot cores/corinos. In fact, in the shocks, dust grains are sputtered and vaporized releasing the mantle components and part of their refractory material into the gas phase. Moreover, shocked regions become hot enough to allow neutral-neutral reactions to take over and produce complex molecules. In the following, we will only review the studies on the chemical composition of the outflow shocks, leaving out the many and important questions on the physical structure of the shock and the acceleration mechanisms of the jet.

Although several molecular outflows have been observed and mapped in the past three decades, the study of their molecular complexity started much later. Bachiller et al. [22] were the first to show the chemical structure of L1157B1, considered nowadays a prototype for the studies of molecular complexity in molecular outflows. Toward this source, not only relatively simple complex molecules, like methanol, have been detected ([110]), but also molecules considered hot cores/corinos tracers, like methyl formate $\left(\mathrm{HCOOCH}_{3}\right)$, ethanol $\left(\mathrm{C}_{2} \mathrm{H}_{5} \mathrm{OH}\right)$, formic acid $(\mathrm{HCOOH})$ and methyl cyanide $\left(\mathrm{CH}_{3} \mathrm{CN}\right)([16])$. High 
spatial resolution observations show that emission of these species is concentrated in a small region associated with the violent shocks at the head of the outflowing material (107). The presence of COMs in molecular outflows strongly suggests that these species were part of the sputtered icy mantles (as the time elapsed since the shock is too short for any gas-phase route to build up COMs) and provides us with another piece of the puzzle regarding their formation. The abundances normalized to methanol are at least one order of magnitude lower in molecular outflows than in hot corinos.

It is worth noticing the presence of species not even detected in other sources, like the phosphorus nitride $(\mathrm{PN})$, whose abundance is only a few times $10^{-10}$ with respect to $\mathrm{H}_{2}$ (477]). In fact, molecular outflows can be considered, for some aspects, unique laboratories to understand interstellar medium chemistry. For example, hydrogen chloride $(\mathrm{HCl})$ has been recently detected with the Herschel Space Observatory in L1157-B1 ([108). The measured abundance is $3-6 \times 10^{-9}$, practically the same value as in high- and low-mass protostellar envelopes (e.g. 344]) and about 200 times lower than the $\mathrm{Cl}$ elemental abundance. This is a puzzling result, as chemical models predict that $\mathrm{HCl}$ would be the major reservoir of chlorine and observational evidence suggests that L1157-B1 is a shock site where grains are sputtered/vaporized and mantles almost entirely destroyed, as also suggested by the large fraction of silicon found in the gas-phase as $\mathrm{SiO}$. Therefore, the low measured $\mathrm{HCl}$ abundance raises the question "where is chlorine?". It is not in the mantle, but not even in the vaporized refractory material of dust grains where silicates reside. Is then chlorine in a significantly more refractory component than silicates? Which one? All questions that will need more observations to be answered.

\subsection{Water and deuterated water}

Water and deuterated water are special species, because of the hints on the Earth and Solar System formation that they bring ( $\$ 3$ and because water plays a leading role in the thermal and chemical evolution of protostellar envelopes ([95; [133]; 427]). However, since water lines can only be observed from outof-the atmosphere telescopes, the water content in the envelope of solar-type protostars has been estimated only recently.

Water abundance in hot corinos. The first estimates based on the Infrared Space Observatory (ISO) suggested that the water abundance in the hot corino region is only a few times $10^{-6}$ (e.g. 91]). The more recent observations obtained with Herschel, with a much better spatial and spectral resolution, have confirmed that first claim with an increased reliability and in a larger number of sources (252]; 250]; 440]; 113]). If, on the one hand, these observations confirm the old theoretical predictions that water should be abundant in the innermost and warmest regions of the envelopes surrounding Class 0 protostars ([95]; 116]), they also raise the question why the measured water abundance is much lower than that expected, $\sim 10^{-4}$, based on the ice measurements (\$4). Finally, interferometric observations have shown that a compact $\mathrm{H}_{2}^{18} \mathrm{O}$ 


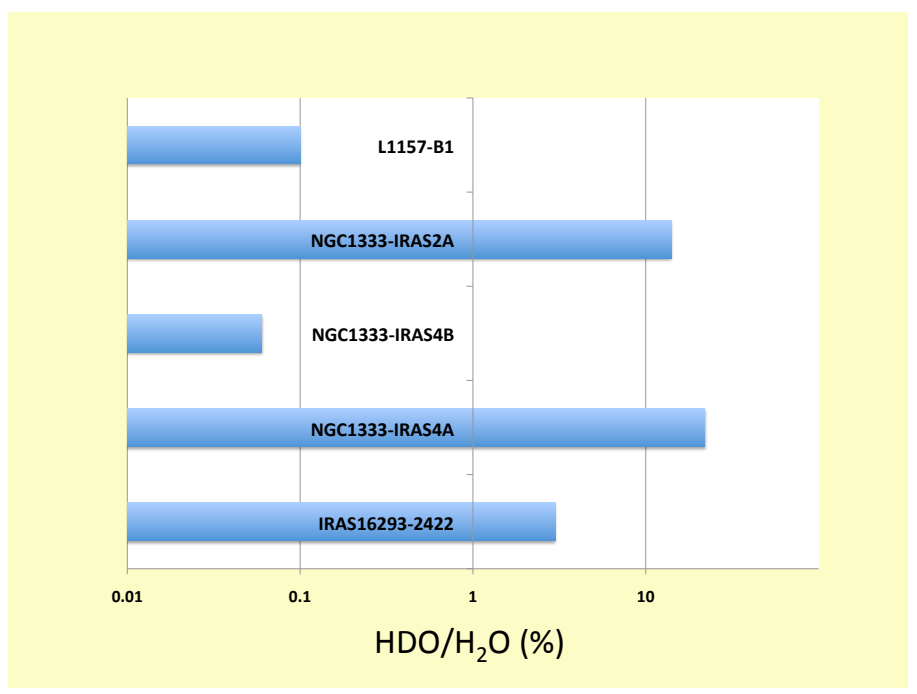

Fig. $10 \mathrm{HDO} / \mathrm{H}_{2} \mathrm{O}$ abundance ration in the envelope of Class 0 protostars and the L1157-B1 outflow shock. References: IRAS16293-2422 ([13), NGC1333- IRAS2A (412]), NGC1333- IRAS4B (234]), NGC1333- IRAS4A (412]), L1157-B1 ([109]). The differences in the $\mathrm{HDO} / \mathrm{H}_{2} \mathrm{O}$ abundance ratio probably reflect the different conditions, density and temperature, at the time when ice mantles formed (see 4 and 7.2 .

emitting region is associated with the hot corinos/disk of a few Class 0 sources $(233] ; 346])$.

Water abundance in molecular outflows. Again, the first estimates of the water abundance in molecular outflows were obtained with ISO and gave abundances varying from $\sim 10^{-5}$ to $\sim 10^{-4}$ ([271; 316]; 31]). Water in outflows was also the target of the Submillimeter Wave Astronomy Satellite (SWAS) and Odin satellite, which were tuned on the $\mathrm{H}_{2} \mathrm{O}$ ground-state transition at $557 \mathrm{GHz}$ (157; 47]; 32]). More recently, the new Herschel observations are providing a mine of new information, allowing us to map the water emission along the outflow and to distinguish the water content in low to high velocity shocks. The Herschel maps show bright water emission at the shock sites of the molecular outflows ([316; 30; 252; 46; 45]). The study of the water abundance as a function of the velocity of the shock then shows that high velocity shocks are associated with larger water abundances ([264]; 251]; 46]; 388]; 431]; 30]), as predicted by the C-shock models (239]). These models predict that $\mathrm{H}_{2} \mathrm{O}$ is formed in the gas-phase via reactions with large activation barriers (e.g. $\mathrm{O}+\mathrm{H}_{2}$ and $\mathrm{OH}+\mathrm{H}_{2}$; see also [211]). Finally, interferometric observations show that the dense shock very close to the central source produces a large quantity of water (266]). 
Deuterated water. The $\mathrm{HDO}$ abundance and $\mathrm{HDO} / \mathrm{H}_{2} \mathrm{O}$ abundance ratio have been measured toward a handful of hot corinos, with different techniques. From single dish telescopes (IRAM 30m and ISO first, then Herschel) the $\mathrm{HDO} / \mathrm{H}_{2} \mathrm{O}$ has been estimated to be $3 \%$ in IRAS16293-2422 ([341; ; 113]) and $\geq 1 \%$ in NGC1333-IRAS2A ([272). Estimates obtained with interferometric observations of $\mathrm{HDO}$ and $\mathrm{H}_{2}^{18} \mathrm{O}$ lines give $\leq 0.06 \%$ in NGC1333- IRAS4B ([233]), and $14 \%$ and $22 \%$ toward NGC1333- IRAS2A and NGC1333- IRAS4A, respectively $(412])$. Note that the interferometric observations provide a direct, almost model-independent, estimate of the $\mathrm{HDO} / \mathrm{H}_{2} \mathrm{O}$ abundance ratio as they do measure the extent of the emission and use the rare $\mathrm{H}_{2}^{18} \mathrm{O}$ isotopologue reducing the problem of line opacity. In summary, the $\mathrm{HDO} / \mathrm{H}_{2} \mathrm{O}$ ratio has been measured toward four hot corinos: in three of them it is larger than a few percent, whereas in NGC1333- IRAS4B it is at least one order of magnitude lower. Herschel observations have also allowed, for the first time, to estimate the $\mathrm{HDO} / \mathrm{H}_{2} \mathrm{O}$ in a molecular outflow shock, L1157-B1 $\left(0.4-2 \times 10^{-3}\right.$, 109]), a likely direct measure of the deuteration in the ice. The situation is summarised in Fig. 10. The differences in the $\mathrm{HDO} / \mathrm{H}_{2} \mathrm{O}$ abundance ratio probably reflect the different conditions, density and temperature, when the ice was formed (see $\$$ and $\$ 7.2$.

Doubly deuterated water. Although it has a very low abundance, $\mathrm{D}_{2} \mathrm{O}$ has an important diagnostic power as it sets very tight constraints to models of water formation. So far, thanks to Herschel, $\mathrm{D}_{2} \mathrm{O} / \mathrm{H}_{2} \mathrm{O}$ has been measured only toward the cold envelope of IRAS16293-2422, with the observations of both the para and ortho forms of $\mathrm{D}_{2} \mathrm{O}([73]$; 432] $)$. The $\mathrm{D}_{2} \mathrm{O} / \mathrm{H}_{2} \mathrm{O}$ abundance ratio results to be $1-4 \times 10^{-3}([113])$. Similarly, the para- $\mathrm{D}_{2} \mathrm{O} / \mathrm{H}_{2} \mathrm{O}$ toward the hot corino is $\sim 5 \times 10^{-5}$ ([73]). Assuming an ortho-to-para ratio equal to 2 gives $\mathrm{D}_{2} \mathrm{O} / \mathrm{H}_{2} \mathrm{O} \sim 10^{-4}$. For example, comparison with the model by 413 . indicates that the bulk of water was formed on grains when the cloud/envelope temperature was $10 \mathrm{~K}$ and the density between $10^{4}$ and $10^{5} \mathrm{~cm}^{-3}$. In other words, when the density at the center of the IRAS16293-2422 pre-stellar cloud reached $10^{6} \mathrm{~cm}^{-3}$, the oxygen not locked into $\mathrm{CO}$ was almost entirely already converted into water.

\subsection{Deuteration of other species}

As water, several molecules present large deuteration factors in low mass protostellar envelopes and molecular outflows (e.g. 342 and 109] respectively). Figure 11 presents a graphic summary of the observations of species with detected doubly or triply deuterated isotopologues. The deuterated ratios are extremely high, with enhancements of the $\mathrm{D} / \mathrm{H}$ of up to 13 orders of magnitude with respect to the elemental D/H abundance ratio. Given the conditions in the envelopes of the protostars $(\$ 5.1$ and Fig. 7 , the observed deuteration is mostly an inherited product of the pre-stellar phase (\$4). Furthermore, for the typical physical condition where the deuterated molecules have been de- 


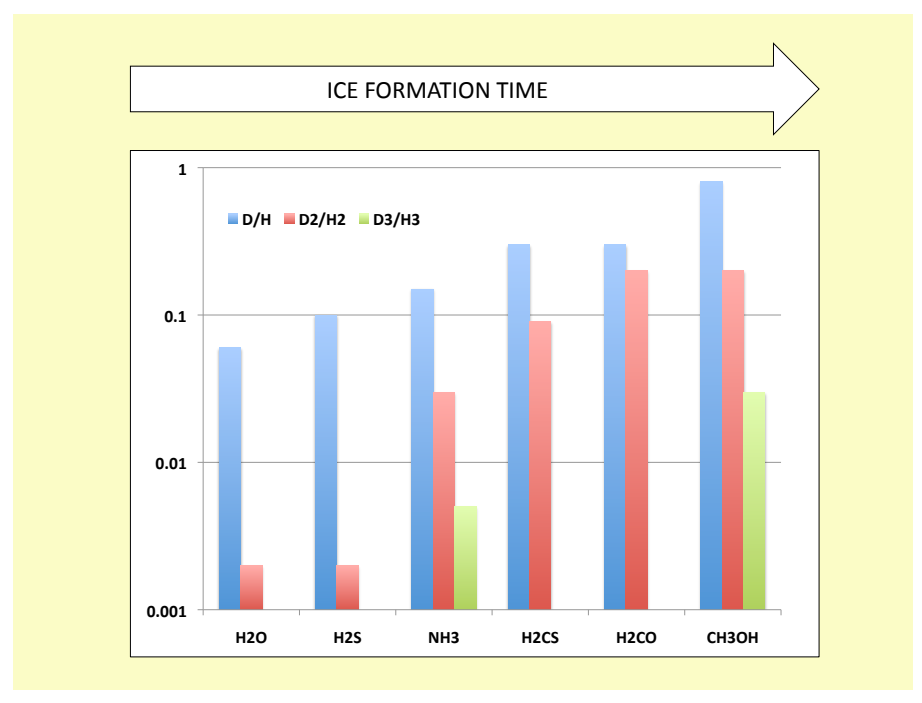

Fig. 11 Measured deuteration ratios of singly, doubly and triply deuterated isotopologues. Based on the modelling of the formation of $\mathrm{H}_{2} \mathrm{O}, \mathrm{H}_{2} \mathrm{CO}$ and $\mathrm{CH}_{3} \mathrm{OH}(86]$; 413 ) we speculate that the increasing deuteration reflects the formation time of the species on the ices. References: $\mathrm{H}_{2} \mathrm{O}$ : [272], [113, [412, [73], 432; $\mathrm{H}_{2} \mathrm{~S}$ : [433] $\mathrm{NH}_{3}$ : 273, 426]; $\mathrm{H}_{2} \mathrm{CS}$ : 282]; $\mathrm{H}_{2} \mathrm{CO}$ : 92]; 342]; $\mathrm{CH}_{3} \mathrm{OH}: 343$, 340, 342].

tected, the measured deuteration ratios likely reflect the deuteration on the grain mantles (e.g. [101]).

We emphasize here that the deuteration ratio is not the same for all species. As mentioned in 4 , the lower deuteration ratio of water with respect to formaldehyde and methanol probably reflects the different epoch in which the bulk of the iced species has been formed (during the pre-stellar phase). Specifically, water is (mostly) formed before formaldehyde, and methanol is the last in the sequence ( 86 ; 410; 413$])$. Even though not specific modelling has been published for all observed deuterated species, we speculate that the sequence in the figure represents a temporal sequence of the species formation.

Finally, the comparison between the singly and doubly deuterated isotopologues provides some interesting additional information. First, if the deuterium atoms were purely statistically distributed, namely just proportional to the $\mathrm{D} / \mathrm{H}$ ratio, then it would hold: $\mathrm{D}$-species $/ \mathrm{D}_{2}$-species $=4(\mathrm{D}$-species $/ \mathrm{H}$ species $)^{-1}$. As shown in Fig. 12, this is not the case for the measured deuteration of $\mathrm{H}_{2} \mathrm{O}, \mathrm{NH}_{3}, \mathrm{H}_{2} \mathrm{CS}$ and $\mathrm{H}_{2} \mathrm{CO}$. As also noted by [3], this points to a change of the atomic $\mathrm{D} / \mathrm{H}$ ratio during the formation of those species or to an origin from gas phase reactions. On the contrary, the statistical relation is roughly valid for $\mathrm{H}_{2} \mathrm{~S}$ and $\mathrm{CH}_{3} \mathrm{OH}$. This suggests that these two species have been formed on the grain surfaces in a very short time, when the atomic $\mathrm{D} / \mathrm{H}$ ratio can be considered roughly constant. 


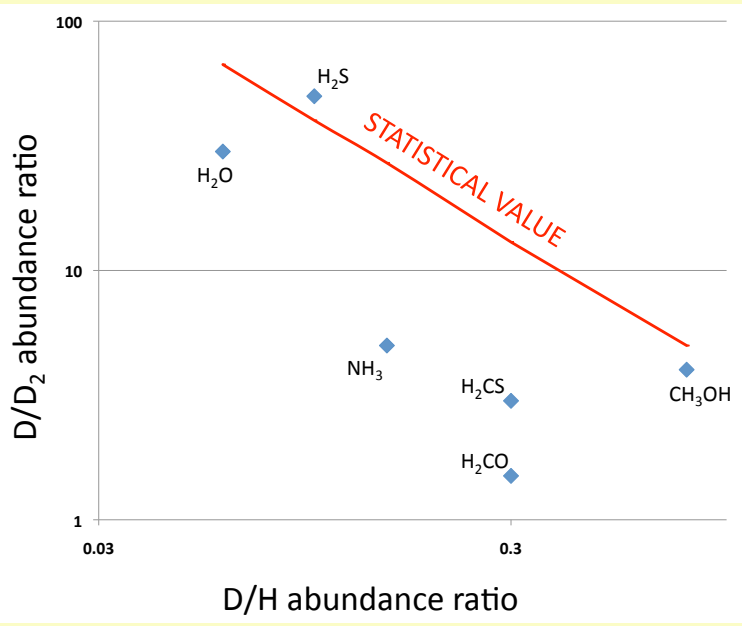

Fig. 12 Measured ratios of singly to doubly deuterated isotopologues of the species marked in the plot. References as in Fig. 11

In summary, the observed deuteration ratios tell us that $\mathrm{H}_{2} \mathrm{O}, \mathrm{NH}_{3}, \mathrm{H}_{2} \mathrm{CS}$ and $\mathrm{H}_{2} \mathrm{CO}$ were formed at various stages during the star formation process, with different values of the atomic gas $\mathrm{D} / \mathrm{H}$ ratio. On the other hand, $\mathrm{H}_{2} \mathrm{~S}$ and $\mathrm{CH}_{3} \mathrm{OH}$ were formed in a shorter time range. This behavior roughly agrees with the models of the formation of $\mathrm{H}_{2} \mathrm{O}, \mathrm{H}_{2} \mathrm{CO}$ and $\mathrm{CH}_{3} \mathrm{OH}$ on grains, that predict that methanol is only formed at very late time, whereas water and formaldehyde are formed over a larger period of time ( 86 ; 410; 411]). It is however possible that the species not close to the statistical value are, at least in part, gas-phase products.

As a final remark, it is important to emphasize that low- and high-mass protostellar envelopes present important differences in the molecular deuteration. A clear example is provided by the $\mathrm{CH}_{2} \mathrm{DOH} / \mathrm{CH}_{3} \mathrm{OD}$ abundance ratio, which is at least one order of magnitude larger in low-mass than in high-mass protostellar envelopes ([366]; 345]).

\section{Toward planet formation: protoplanetary disks}

Starless and pre-stellar cores present evidence of overall (slow) rotation ([20]; [181; [78]), thus they possess an initial angular momentum. As a natural consequence of angular momentum conservation, the collapse of pre-stellar cores produces flattened structures which harbor the future protoplanetary disks. Even non-rotating collapsing cores are expected to produce flattened structures in the presence of magnetic fields, as explained in the following. As 
ionized particles within the core are linked to the magnetic field lines, while neutrals only feel the gravitational field, a drag between ions and neutrals is established during the collapse phase (see \$4). Galli \& Shu ([165; ; 166]) found that during the collapse of a singular isothermal sphere (i.e. an unstable spherical cloud with a density profile proportional to $r^{-2}$, thus with a singularity in the center, [398]), the magnetic field, dragged by the flow, deflects the infalling gas toward the midplane, forming a large ( $\simeq 2000 \mathrm{AU})$ "pseudodisk". The magnetic field lines, initially parallel, are shaped as an hourglass, consistent with observations of polarization maps of the dust continuum emission toward young stellar objects (e.g. 177]; 158]). The twisting of magnetic field lines in the pseudodisk acts as a "magnetic break", in the sense that it slows down the rotation by transferring angular momentum from the inner regions (which tend to rotate faster for angular momentum conservation) of the pseudodisk toward its outer parts ([28]). Indeed, magnetic breaking is so efficient, that disks cannot form at all in ideal magneto-hydrodynamic (IMHD ${ }^{4}$ simulations of collapsing cores (e.g. 11]; 296]; 200]). More recently, the inclusion of non-ideal MHD effects, in particular the Hall effect $t^{5}$ (65]; 249]), has helped to avoid this so-called magnetic breaking catastrophe, allowing disks of about $100 \mathrm{AU}$ to form (even without initial rotation of the collapsing cloud, 65]). This has also been shown in simulations by 276], who found rapid growth to $\geq 100 \mathrm{AU}$ of the circumstellar disk when depletion of the infalling envelope is taken into account, and by [227, who explored the case of magnetic fields non-aligned with the rotation axis and found less efficient angular momentum transport, allowing the formation of $\simeq 100-200 \mathrm{AU}$ disks, with masses as large as $10 \%$ the original core mass. These characteristics are similar to the young self-gravitating protoplanetary disks (we refer to them as "embedded disks") which can become gravitationally unstable (e.g. 258; 59]; 140 and references therein; [55]; 443]) and which represent the starting point of our final journey toward the formation of a planetary system. Here we will focus on the chemical evolution (see [18] and [463] for comprehensive reviews on the physical characteristics and evolution of protoplanetary disks).

\subsection{Embedded disks: chemistry at the dawn of planet formation}

Young disks are embedded within the thick and massive envelopes of Class 0 sources (see \$5). Therefore, they are not easy to study and it is hard to put constraints on theoretical predictions. Indirect evidence of young disks in Class 0 sources is given by the presence of collimated outflows, observed with millimeter and sub-millimeter telescopes $(\$ 5$ ). ALMA will of course revolutionize this

\footnotetext{
4 IMHD assumes that the mass to magnetic-flux ratio is constant, which implies that magnetic field lines follow the gas motions, i.e. the magnetic field is "frozen" into the neutral medium.

5 The Hall effect mainly operates at volume densities between $10^{8}$ and $10^{11} \mathrm{~cm}^{-3}$ ([451]), where the more massive charged particles (ions and charged dust grains) decouple from the magnetic field and collisionally-couple with the neutral gas.
} 


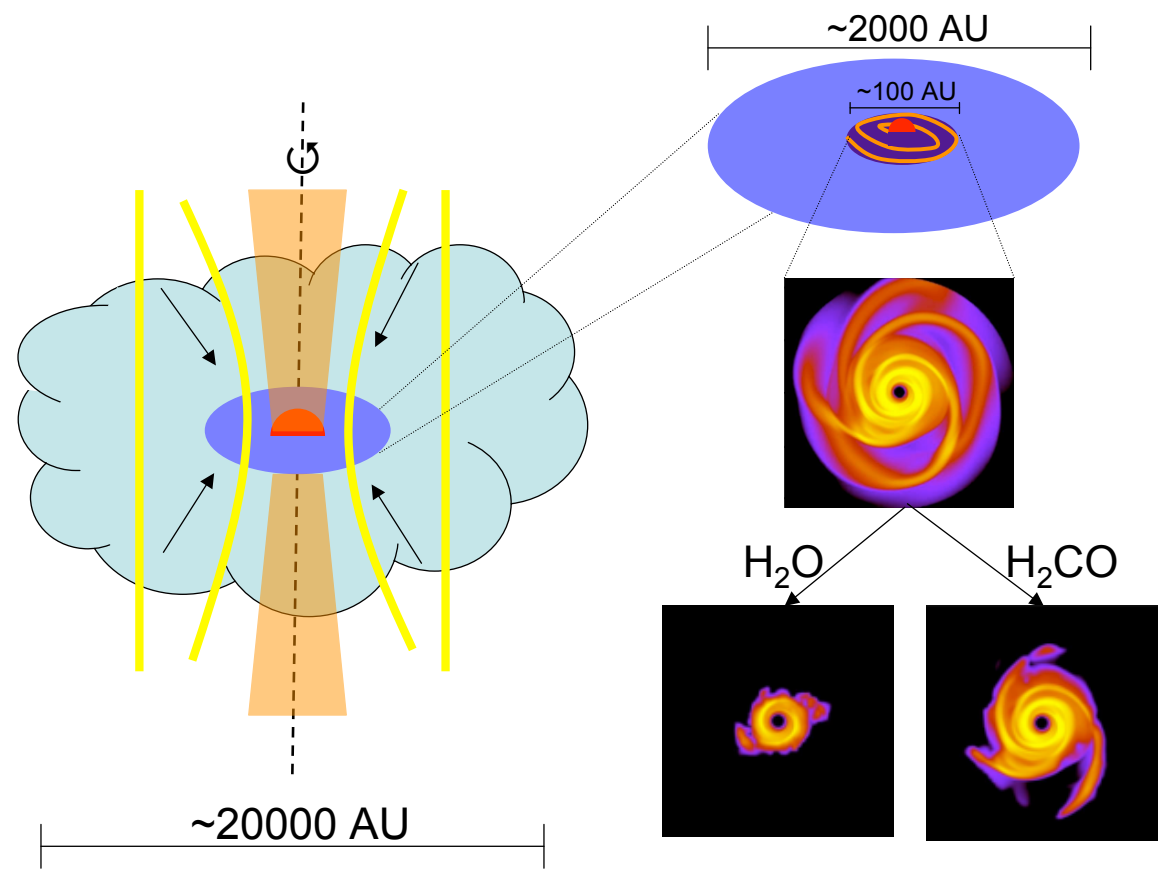

Fig. 13 The earliest stages of a protoplanetary disk. Magnetic fields (yellow curves) and the initial rotation of the pre-stellar core lead to the formation of a flattened structure (the "pseudodisk", size $2000 \mathrm{AU}$ ) surrounding the accreting protostar. In the central few hundred $\mathrm{AU}$, the embedded disk can be self-gravitating, develop spiral structure and experience fragmentation. Molecules such as $\mathrm{H}_{2} \mathrm{O}$ and $\mathrm{H}_{2} \mathrm{CO}$ are good tracers of these central regions of young embedded disk (217]).

field. After the pioneer work by, e.g., [97, 68] and [275], further steps toward the characterization of these embedded disks have been made by [228, 235] and [147. With the help of interferometric observations (able to filter out the surrounding envelopes), these authors found evidence of compact embedded disks in Class 0 sources, with masses ranging from 0.04 to $1.7 \mathrm{M}_{\odot}$. Choi et al. ([105]) observed $\mathrm{NH}_{3}$ with the Very Large Array (VLA) and found a $130 \mathrm{AU}$ circumstellar disk around NGC1333 IRAS4A2. With the IRAM Plateau de Bure Interferometer (PdBI), 234] measured water vapor $\left(\mathrm{H}_{2}^{18} \mathrm{O}\right)$ in the inner $25 \mathrm{AU}$ of the NGC1333 IRAS4B disk, suggesting the presence of a thin warm layer containing about 25 Earth masses of material. Toward the same object, 233] also set a stringent upper limit on the $\mathrm{HDO} / \mathrm{H}_{2} \mathrm{O}$ abundance ratio to $6 \times 10^{-4}$ (\$5). Pineda et al. ([350]) observed methyl formate with ALMA toward IRAS16293-2422, a binary Class 0 source in Ophiuchus (\$5), and found the first evidence of infall toward source B and evidence of rotation toward source A, consistent with an almost edge-on disk (see also [380]). If confirmed, this could be the first chemically and kinematically characterized embedded disk (discovered with a complex organic molecule!). 
What are the chemical model predictions of these embedded disks? Visser et al. (441, 438]) have been the first to self-consistently follow the chemistry in a two-dimensional axisymmetric model of a collapsing (initially) spherical and slowly rotating cloud, on its way toward the formation of a protoplanetary disk. The material infalling in the equatorial plane, within the centrifugal radius 6 forms the disk, whose evolution is also considered assuming no mixing. The disk-envelope boundary and the outflow cavities are well defined. Detailed predictions are given about the ice and gas-phase composition of the cloud-disk system at different evolutionary phases. At the end of the collapse phase, they find that disks can be divided in zones with different chemical history, which will ultimately affect the composition of comets formed in different zones. Different results are found by [217, who used the hydrodynamic simulations of a young and relatively massive $\left(0.39 \mathrm{M}_{\odot}\right)$ disk by [54] as input in their gas-phase and simple surface chemistry network. Boley's disk resembles in mass and size the embedded disk mentioned above, it is non-axysimmetric and present complex spiral and physical structure, with shocks moving with the spirals arms (see Figure 13, middle panel in the left, which reports the gas column density map). No accretion of material from the envelope and no outflow is considered. Despite these assumptions, the disk structure is complex and its physical characteristics are continuously stirred by the rotating spiral arms. Because of this continuous mixing, 217 found no separated chemical zones as in the case of [438, but they identified species able to trace the inner regions of the disk (such as $\mathrm{H}_{2} \mathrm{O}$, $\mathrm{HNO}$ and $\mathrm{NH}_{3}$ ) and those tracing the spiral arms (e.g. $\mathrm{H}_{2} \mathrm{CO}$ and $\mathrm{HCO}^{+}$). Examples of these column density maps are given in Fig. 13 (bottom right panels), which also summarizes the various physical mechanisms to be considered for a comprehensive study of the earliest stages of star formation: the collapsing envelope of a Class 0 source (red semicircle) under the influence of magnetic fields (yellow lines and curves in the figure), the pseudodisk (blue), the central embedded disk (violet) and the outflow (orange) driven by the central protostar (red semicircle). Furuya et al.([164]) studied the chemical evolution of a molecular core toward the formation of the first hydrostatic core (protostellar precursor) using three-dimensional radiation hydrodynamic simulations. They show that after a first destruction of molecules, simple species such as $\mathrm{CO}, \mathrm{H}_{2} \mathrm{O}$ and $\mathrm{N}_{2}$ reform and more complex molecules $\left(\mathrm{CH}_{3} \mathrm{OH}\right.$ and $\left.\mathrm{HCOOCH}_{3}\right)$ can trace the first hydrostatic core, on its way to becoming a protostar. ALMA observations are needed to disentangle the various phenomena at work during the earliest stages of star formation, to test model predictions of collapsing magnetized pre-stellar cores and to unveil the physical and chemical structure of the embedded disks, precursors to the protoplanetary disks which will be reviewed in the next sections. 


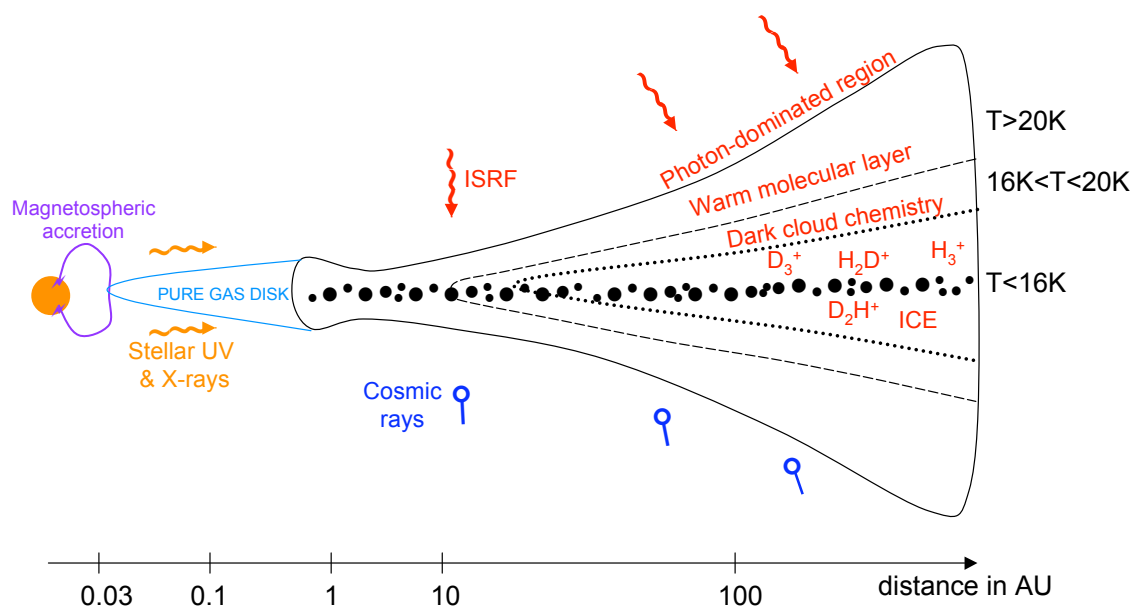

Fig. 14 Schematic structure of a "naked" protoplanetary disk, adapted from 324, 139, [395, 138 and 37. The various regions are labeled. The black dots with various sizes represent the coagulated dust in the disk midplane. See text for details.

\section{2 "Naked" protoplanetary disks}

The embedded phase of disks does not last long. After about $0.5 \mathrm{Myr}$ since the birth of the protostar/disk/outflow system, the parent core envelope quickly disperses and the disk enters a new phase which lasts several Myr (463]). This is the T Tauri (or Class II) phase. The disk mass is now only a few $\%$ the stellar mass ([463]) and the motions are expected to be Keplerian. Despite being "naked" disks, thus easier to observe than during the earlier embedded phase, the physical and chemical processes at work are complex and more (interferometric) data are sorely needed to fully understand them. Figure 14 shows a schematic picture of a T Tauri disk, compiled from a combination of figures found in [324, 139, 395, [138] and 37]: (1) within the central $1 \mathrm{AU}$ from the star, a pure gas disk and the dust inner rim are present. This zone is mainly probed by $\mathrm{Br}-\gamma$ lines (e.g. 311]; 277; ; 414; ; 184]), $\mathrm{H}_{2}$ ([36; 156]), as well as near-infrared lines of $\mathrm{CO}, \mathrm{H}_{2} \mathrm{O}, \mathrm{OH}(386$; [77] ; 358] ) and simple organic molecules ([278]). (2) Moving away from the central star, one finds the "puffed-up" inner dust wall ([313]), clearly seen in the near-infared continuum, where the higher temperature affects its vertical scale height, which is set by hydrostatic equilibrium. Within the central few $\mathrm{AU}$, mid-infrared emission of $\mathrm{H}_{2} \mathrm{O}, \mathrm{CO}$ and the organic molecules $\mathrm{HCN}$ and $\mathrm{C}_{2} \mathrm{H}_{2}$ have been measured ([256]; 77]). Carr \& Najita (77]) note that the $\mathrm{HCN} / \mathrm{H}_{2} \mathrm{O}$ abundance ratio is largest in the most massive disks and speculate that this may be indication of the sequestration of $\mathrm{H}_{2} \mathrm{O}$ in the outer disk during the process of planetesimal formation. It is interesting to note that toward the disks surrounding the intermediate mass $\left(\simeq 2<\mathrm{M} / \mathrm{M}_{\odot}<8\right)$ Herbig Ae/Be stars, no

6 The radius at which the gravitational force is balanced by the centrifugal force. 
organic molecules have been detected ([359]; 387]) and water is only seen in the far-infared at larger radii ( $\simeq 15-20 \mathrm{AU}$; 149$]$ ), probably due to the larger UV fluxes compared to T Tauri stars. Beyond the "wall", the disk is thought to have a layered structure. (3) A photon-dominated region (PDR) is present all around the disk, which is exposed to the stellar and interstellar UV field, as well as the stellar X-rays. Here, forbidden line emission from the well-known PDR coolants, [CII]158 $\mu \mathrm{m},[\mathrm{OI}] 63 \mu \mathrm{m}$ and $145 \mu \mathrm{m}$, are observed ([407] 356]), although the [CII] $158 \mu \mathrm{m}$ and the [OI]145 $\mu \mathrm{m}$ are not always detected (287]; 417]). (4) A warm molecular layer. Just below the PDR zone, molecules survive, although photochemistry is still playing an important role ([201; ;17]). The gas and dust are warm and radical and ions dominate the gas composition ([395]). (5) A dark-cloud chemistry zone, where the temperature drops below $20 \mathrm{~K}$, molecular freeze-out becomes important and simple species typically found in dark clouds are detected: $\mathrm{CO}$ isotopologues with evidence of depletion (141; 143 ; 362]), CN, HCN, HNC, CS, HCO ${ }^{+}, \mathrm{C}_{2} \mathrm{H}$ and $\mathrm{H}_{2} \mathrm{CO}$ ([142; 430; 418; 99]), $\mathrm{N}_{2} \mathrm{H}^{+}$(144]), SO ([162]), CS ([145]), $\left.\mathrm{DCO}^{+}(428]\right)$, $\mathrm{H}_{2} \mathrm{D}^{+}([94])$, $\mathrm{HDO}\left(\left[93\right.\right.$, but see [190]), $\mathrm{HC}_{3} \mathrm{~N}([98])$. Qi et al. ([363]) spatially resolved the emission of $\mathrm{DCO}^{+}$and measured the deuterium fraction across the disk of TW Hydrae, finding a range between 0.01 and 0.1 , with a peak around $70 \mathrm{AU}$. They also measured the DCN/HCN abundance ratio, $\simeq 0.02$, similar to that measured in the jets of material coming from the nucleus of comet Hale-Bopp ([294]). Öberg et al. ([322]) used the Sub-Millimeter Array (SMA) to image disks of six Taurus sources with spectral type from M1 to A4, finding similar intensities of $\mathrm{CN}$ and $\mathrm{HCN}$ lines in T Tauri and Herbig Ae stars, but a significantly different chemical richness: deuterated molecules, $\mathrm{N}_{2} \mathrm{H}^{+}$and $\mathrm{H}_{2} \mathrm{CO}$ were only detected toward T Tauri star disks, implying a lack of long-lived cold regions in the disks of the more massive Herbig Ae stars (see also 323]). Water vapor in the cold outer disk has been detected toward TW Hydrae by [209] with Herschel, revealing a hidden large reservoir of water ice at large radii (between 100 and $200 \mathrm{AU}$ ). Indeed, ice features have been detected in the direction of edge-on protoplanetary disks by [415] and 213. More recently, 4 measured with the AKARI satellite several ice features in edge-on Class II disks, including a faint HDO feature, which allowed them to measure a solid $\mathrm{HDO} / \mathrm{H}_{2} \mathrm{O}$ abundance ratio between $2 \%$ and $22 \%$ (significantly larger than the $\mathrm{HDO} / \mathrm{H}_{2} \mathrm{O}$ ratio measured in comets and in star-forming regions; see $\$ 3$ and $\$ 5$. (6) The midplane, characterized by cold and dense regions, with large amounts of molecular freeze-out, where only light species can survive ([324]), in analogy with the central $\simeq 1000$ AU of pre-stellar cores (\$4).

Several chemical models of this protoplanetary disk phase, with various degrees of complexity, have been developed: X-ray chemistry ([178]; 295]; 406]), surface chemistry (e.g. [460]), accretion flows ([6]; 218]), thermal balance ([182]), grain growth ([5]; 436]), UV continuum and Ly $\alpha$ radiation ([35]; 154]), turbulence-driven diffusion (476]; 459]), viscous accretion, turbulence mixing and disk winds ([204]; 199]), photochemistry and wavelength-dependent reaction cross sections, (448), comprehensive physical, chemical and radiative transfer modeling ([183]; 467]; 238]). Despite the advances in chemical com- 
plexity, large uncertainties are still present on several reaction rates (435]) and collisional coefficients, so that laboratory studies and theoretical investigations are still sorely needed to improve the reliability of modern astrochemical models. Moreover, the large uncertainties in the process of dust evolution and coagulation in disks are also shaking our understanding of the disk chemical structure. Laboratory experiments (e.g. 191]; 394]), numerical simulations (e.g. 482]) and theoretical work (e.g. 131; 465]) are fundamental to progress in this field and an effort has to be made to link dust coagulation models with astrochemistry.

As schematically shown in Fig.14, in the midplane the dust settles and coagulates with its thick icy mantles and larger grains tend to settle first (e.g. 137). The differential dust settling and the presence of some degree of turbulence mixing, maintains a population of small dust grains in the upper layers of the disk (see also 124). This includes polycyclic aromatic hydrocarbons (PAHs), ubiquitous in active star-forming regions (422) and also present in protoplanetary disks, especially around the intermediate-mass Herbig $\mathrm{Ae} / \mathrm{Be}$ stars (e.g. 193]; 1]; 242]; see also 237] for a recent review of PAH in disks). PAH features have been detected in only $8 \%$ of the less massive T Tauri stars ([173). PAHs are not only important from an organic and pre-biotic chemistry point of view, but also for the physical structure of disks, as they can be photoionized, releasing energetic photons which heat the gas, thus maintaining flared disk structures (237). Moreover, PAHs boost the formation of $\mathrm{H}_{2}$ molecules ([192]), thus the atomic-to-molecular transition in the upper disk atmospheres. Habart et al. ([194]) spatially resolved the $3.3 \mu \mathrm{m}$ PAH feature toward Herbig Ae/Be stars, finding that the emission originates from within $30 \mathrm{AU}$ of the star. In T Tauri stars, the less intense stellar UV field makes the detection of PAH features more difficult (as PAH features are excited by photons). Visser et al. (439]) predict that PAHs in T Tauri disks can survive much closer to the star (down to about $0.01 \mathrm{AU}$ for a 50-carbon atoms $\mathrm{PAH}$ ) compared to the Herbig disks (down to $5 \mathrm{AU}$ for PAHs with 96 carbon atoms). However, 402] include the effects of extreme UV and X-ray components in their models and find very efficient PAH destruction also in T Tauri stars; by taking into account typical X-ray luminosities, 401, are able to reproduce the different PAH detection probabilities observed in T Tauri and Herbig Ae disks. Fedele et al. ([150]) found PAH emission co-spatial with the [OI]63 $\mu \mathrm{m}$ line, i.e. in the photon-dominated zone of the disk of a Herbig star. As UV photons can break the weaker $\mathrm{C}-\mathrm{H}$ bonds in PAHs and their carbon skeleton can also brake above a certain threshold of energy intake ([189]), the presence of PAHs in the upper atmosphere of disks hints at some replenishing mechanism, possibly vertical mixing (401]), which maintains a population of small grains mixed with the gas ([137]). Habart et al. (193]) suggest that the observed PAHs are evaporated from the icy grain mantles within the disk, while others consider them as the result of fragmentation of larger grains ([365]). The mixing of PAHs within the icy mantles of dust grains, could provide an interesting starting point for the formation of more complex molecules, once dust grains start to coagulate and form larger bodies (64; 63]). 
6.3 From debris to icy worlds

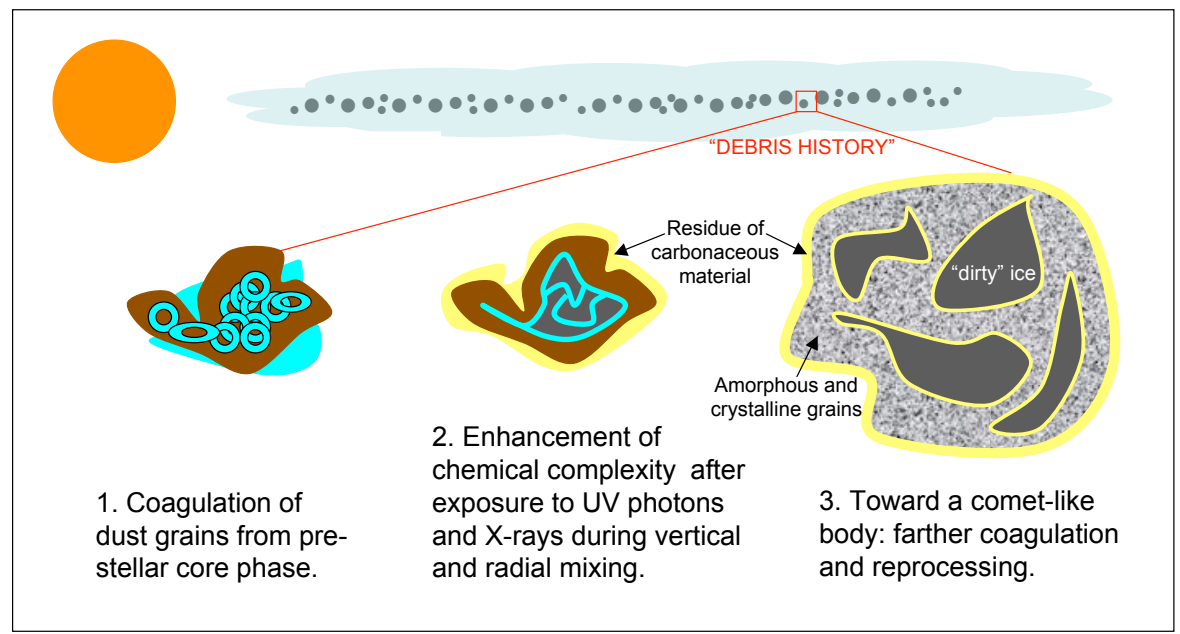

Fig. 15 Sketch of a debris disk and a speculative history of a debris: 1. Dust grains with their icy mantles (blue rings) coagulate and form a fluffy structure, on top of which more ice can adsorb/form; 2. Exposure to UV photons and X-rays changes the inner structure of the grain and allows the surface icy mantle to be reprocesses and form a refractory carbonaceous material; 3. Further coagulation will lead to small rocks, composed by a mixture of the fluffy grains in 1 \& 2 with their refractory organic material and "dirty" ice, glued together by a mixture of amorphous and crystalline dust material.

The transition between protoplanetary disks and planetary system is far from being understood. As 463 pointed out, "exactly how and when protoplanetary disks evolve into planetary debris disks remains an open question". In protoplanetary disks, there is plenty of evidence of dust grain growth ([29]; [122]; 468]; 416]; 464]; 12]; 223]; 112]; 274]; 262]), dust settling ([135]; [74]; [123] ; 163]), dust processing (e.g. presence of crystalline silicates; 244]; 314]; 389]; 297]; 330]; 373]), inner holes (probably carved by a planet or by photoevaporation, [75]; 215]; 14]; 13]; 106]). Debris disks are also observed, with their poor gas content and with evidence of large grains and/or planets (475]; 216; 374]). Despite all these measurements, the story behind grain growth and planetesimal formation remains obscure (see also previous sub-section). For example, one of the biggest challenges for planet formation theories is the so-called "meter-size barrier", where models show destructive collisions and rapid inward migration of meter-sized solids ([454]; 463]). Nevertheless, the presence of large grains in protoplanetary disks and the structure of our Solar System tell us that dust grains coagulate and evolve toward rocks, comets, asteroids, planetesimals, planets and moons. There are connections between the petrology observed in protoplanetary disks and that in our Solar System bodies. In fact, crystalline grains detected in comets (470]; 471]; 481], who 
also suggest aqueous alterations in the comet P81/Wild2) are mostly made out of Mg-rich olivine grains, consistent with observations of gas-rich T Tauri disks. Fe-rich grains have been observed in several interplanetary dust particles (IDPs, e.g. [71]) and recently in warm debris disks ([330]). Such Fe-rich grains may be due to a secondary alteration of the disk mineralogy (see also [315]), probably originated within large differentiated bodies (as in the case of the S-type asteroid recently studied with the Hayabusa re-entry module; 312]). In this scenario, planetesimals form with internal temperatures large enough (from the decay of short-lived radionuclides) to allow the melting and gravitational segregation of silica and metals. Destructive collisions among these planetesimals would then contribute to the production of the Fe-rich particles found in IDPs and in warm debris disks and to the replenishment of small dust grains in our Solar System as well as in exo-zodiacal belts.

Let us now retrace the history of a dust grain during the process of star and planet formation. The starting point has to be found within dense cores, where dust grains have thick icy mantles (see $\$ 4$ and Fig. 6) and show some evidence of coagulation (e.g. 246; 336]), also found soon after protostellar birth, in Class 0 objects (228]; 253]; 103]). As we have seen in previous sections, these dust mantles are rich in water and simple organic material and the chemical complexity in ices appears to increase with dynamical evolution. Figure 15 show a schematic possible scenario of the formation of a debris in the late stages of evolution of protoplanetary disks: (1) soon after the formation of the protoplanetary disk, dust grains coagulate and become fluffy aggregates of the original icy dust grains. They may go through shocks during the early "stirring" of the embedded self-gravitating disks (Fig.13). (2) During the "naked" $\mathrm{T}$ Tauri phase, some vertical and radial mixing may expose the fluffy aggregate to stellar and interstellar UV photons and stellar X-rays, so that icy material on the surface can partially be photodesorbed and partially reprocessed, with the production of radicals and formation of an organic residue on the surface (the yellow layer in the figure) and formation of complex organic molecules in the ice trapped within the aggregate. (3) Further processing and coagulation (including some crystalline dust reformed in the inner parts of the disk) could then lead to a cometary-like body, where "dirty ice" (i.e. ice mixed with complex organic molecules) is a major component.

We are now ready to attempt assembling some pieces of the puzzle.

\section{Putting together some pieces of the puzzle}

7.1 Molecules in comets and solar-type protostars

All molecules detected in comets are also observed in star-forming regions. However, the measured abundances in comets and Sun-like star formation regions are not the same. This is clearly shown in Fig. 16, which reports the abundances, normalized to the methanol abundance, of species detected in various comets (see the reviews [309] and [49], and $\$ 3.2$ ) and those in the 


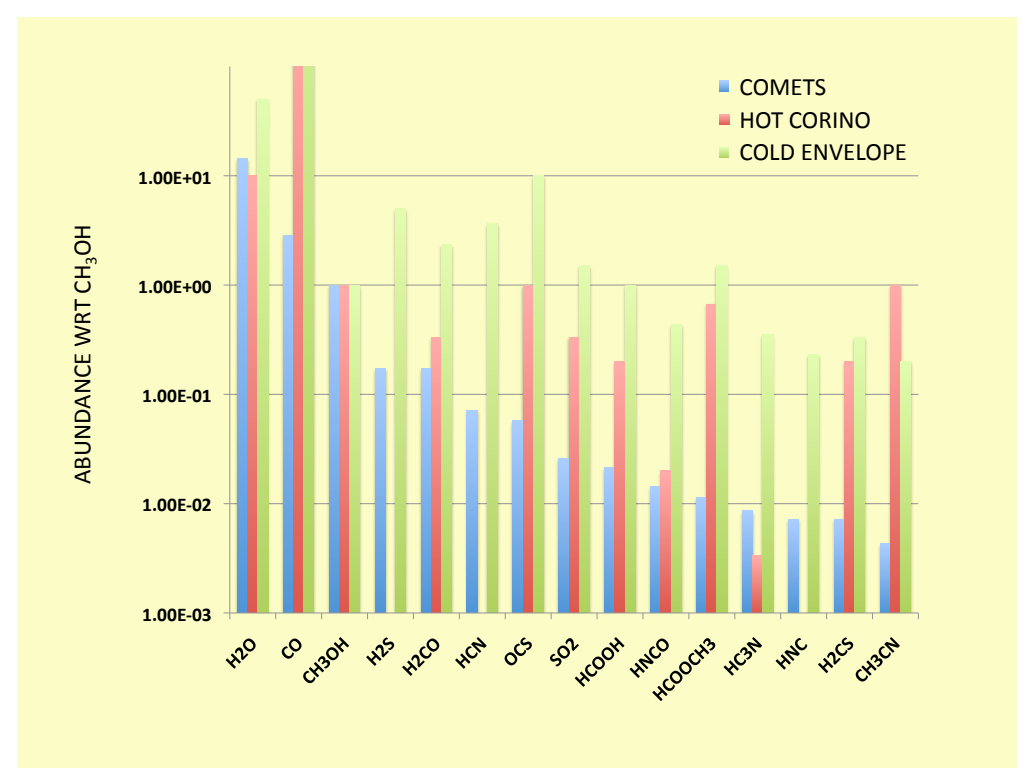

Fig. 16 Abundances (with respect to $\mathrm{CH}_{3} \mathrm{OH}$ ) of molecules detected in comets (blue) and in the hot corino (red) and cold envelope (green) of IRAS16293-2422. References: for comets 309 and 49; for IRAS16293-2422 96, 391] and 89.

hot corino and cold envelope of IRAS16293-2422 (see \$5.1). A similar plot is obtained also if the normalisation is done with respect to water rather than methanol. In general, species are more abundant with respect to methanol (and water) in IRAS16293-2422, both in the cold envelope and the hot corino, than in comets by more than a factor of ten. In other words, the chemistry in comets seems to be less rich than in both the cold envelope and the hot corino of IRAS16293. It is, therefore, probably fortuitous the rough correlation found in the abundance of a fewer molecules in comets and hot cores (e.g. [49]).

Where does this difference come from? The molecules in the cold envelope of IRAS16293-2422 are likely the product of gas-phase chemistry (but see the comments in $\$ 4$ in cold gas, where CO is largely frozen into the grain mantles. Therefore, the systematic difference between the molecular abundances in comets and the cold envelope may point to different physical conditions, likely warmer, at the time of the comet formation. Similarly, the molecules in the hot corino are thought to mostly reflect the composition of the grain mantles during the pre-collapse phase, so that the difference in this case also suggests warmer conditions of the material when the cometary ices were formed. There are, however, also other possibilities. It is possible that the cometary ices have undergone a massive reprocessing of the molecular composition due to the long irradiation from cosmic rays and solar wind particles and UV irradiation. Or it is possible that our Sun's progenitor, in fact, did not resemble the IRAS16293- 


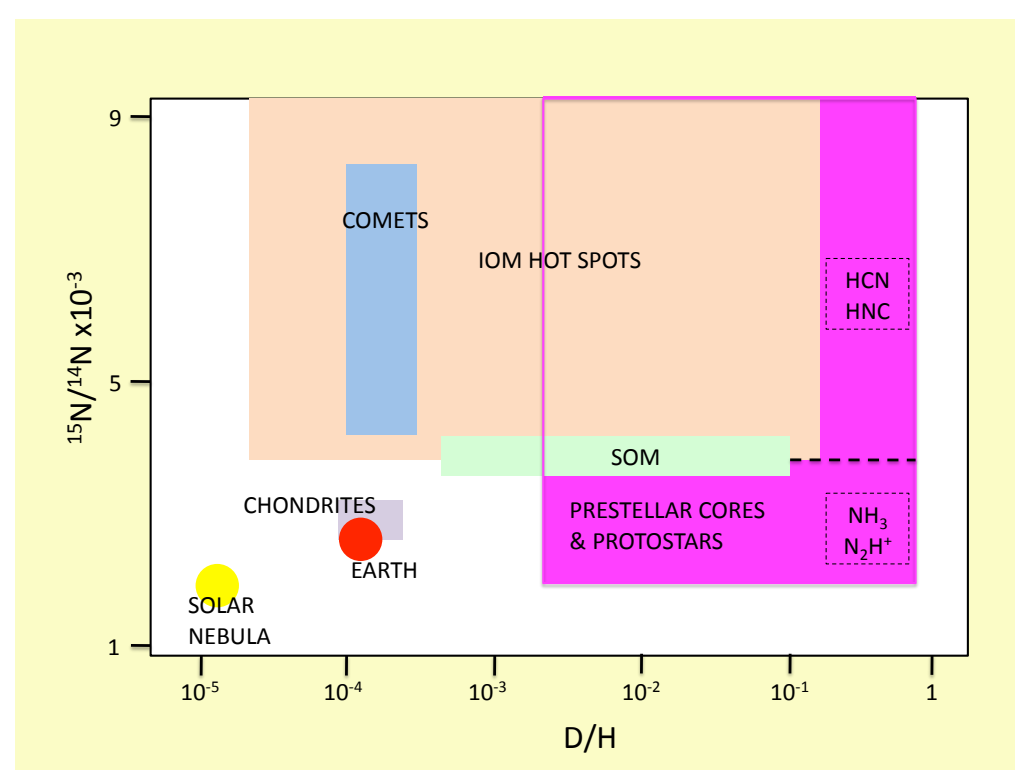

Fig. $17{ }^{15} \mathrm{~N} /{ }^{14} \mathrm{~N}$ versus $\mathrm{D} / \mathrm{H}$ in comets, chondrites, hot spots in the IOM of meteorites and IDPs, SOM in meteorites, Earth, Solar Nebula and pre-stellar cores and protostellar envelopes.

2422 protostar, which is rather isolated, whereas the proto-Sun likely was born in a crowded and much harsher environment (\$3.5). Our census of the molecular composition in comets and in protostellar objects thought to be similar to the proto-Sun is still too poor to have a definitive answer.

\subsection{Origin of deuterated molecules in comets and chondrites}

For a long period it has been though that there is a link between the chemistry in comets, chondrites and interstellar medium, especially because of the enhanced abundance of deuterated molecules (Fig. 17). It is possible that the link is not direct, meaning that it may not be due to the passage of the molecular deuteration from one phase to the next, during the formation of the Solar System. However, the link certainly exists because the chemistry regulating the molecular deuteration is common to all phases and it has to do with the low temperatures occurring during the star and planet formation.

Two key parameters play a major role in the molecular deuteration, regardless of the details which depend on the specific molecule: the ratios of $\mathrm{H}_{2} \mathrm{D}^{+} / \mathrm{H}_{3}^{+}$and of the atomic $\mathrm{D} / \mathrm{H}$ in the gas $(4.1$ and $\$ 5.5$. Figure 18 shows how the $\mathrm{H}_{2} \mathrm{D}^{+} / \mathrm{H}_{3}^{+}$ratio depends on the gas temperature. Another important parameter for this ratio is the abundance of gaseous $\mathrm{CO}$, as $\mathrm{CO}$ is a major destroyer of molecular ions, being the most abundant heavy-atom-bearing 


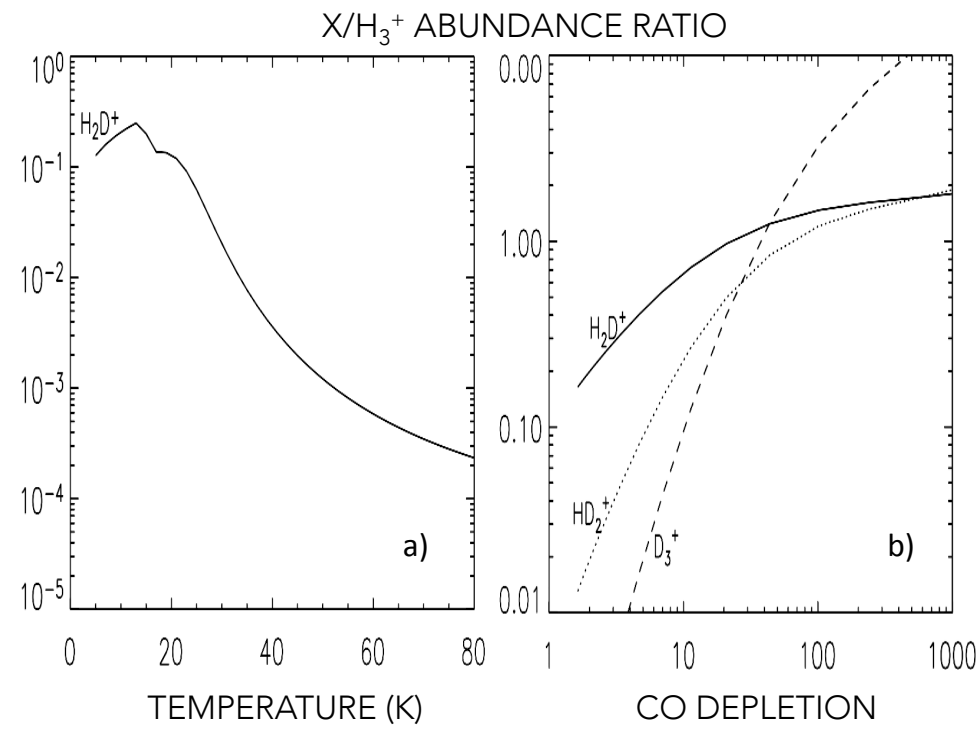

Fig. 18 Theoretical abundances of the $\mathrm{H}_{3}^{+}$isotopologues in the gas. The left panel (a) reports the plot of the ratio $\mathrm{H}_{2} \mathrm{D}^{+} / \mathrm{H}_{3}^{+}$as function of the gas temperature, for gas with no $\mathrm{CO}$ depletion. The right panel (b) shows the abundance ratio of all the $\mathrm{H}_{3}^{+}$isotopologues as a function of $\mathrm{CO}$ depletion, for a gas with temperature $10 \mathrm{~K}$. In both cases, the gas density is assumed $10^{5} \mathrm{~cm}^{-3}$ (adapted from Ceccarelli \& Dominik 2005).

neutral molecule. In cold and dense regions, CO may freeze-out onto the grain mantles and disappear, therefore, from the gas-phase (4.1). Figure 18 also shows the dependence of the $\mathrm{H}_{2} \mathrm{D}^{+} / \mathrm{H}_{3}^{+}$and the other isotopologues of $\mathrm{H}_{3}^{+}$as a function of the $\mathrm{CO}$ depletion, namely how much the $\mathrm{CO}$ abundance is reduced with respect to the standard molecular cloud value. In general, molecular deuteration exceeding $10 \%$ requires not only cold gas but also a substantial drop of the $\mathrm{CO}$ abundance in the gas-phase.

Similarly, Fig. 19 shows how the gaseous D/H ratio, which governs the molecular deuteration of grain-surface product molecules $(4.2)$, varies with the $\mathrm{CO}$ depletion in different situations. Also in this case, large $(\geq 10 \%)$ molecular deuteration can only be achieved in cold gas deprived of CO.

Therefore, the relatively low water deuteration measured on Earth, in comets and in chondrites with respect to the values measured in the prestellar and protostellar phases suggest substantial remixing of water ices since their first formation. How much of the first ices remains in the terrestrial, cometary and chondritic water is difficult to say but cannot be substantial. On the contrary, the extremely large deuteration found in the chondritic organic material, both soluble and insoluble, testifies either the preservation of molecular species since the very first stages (where not only the temperature 


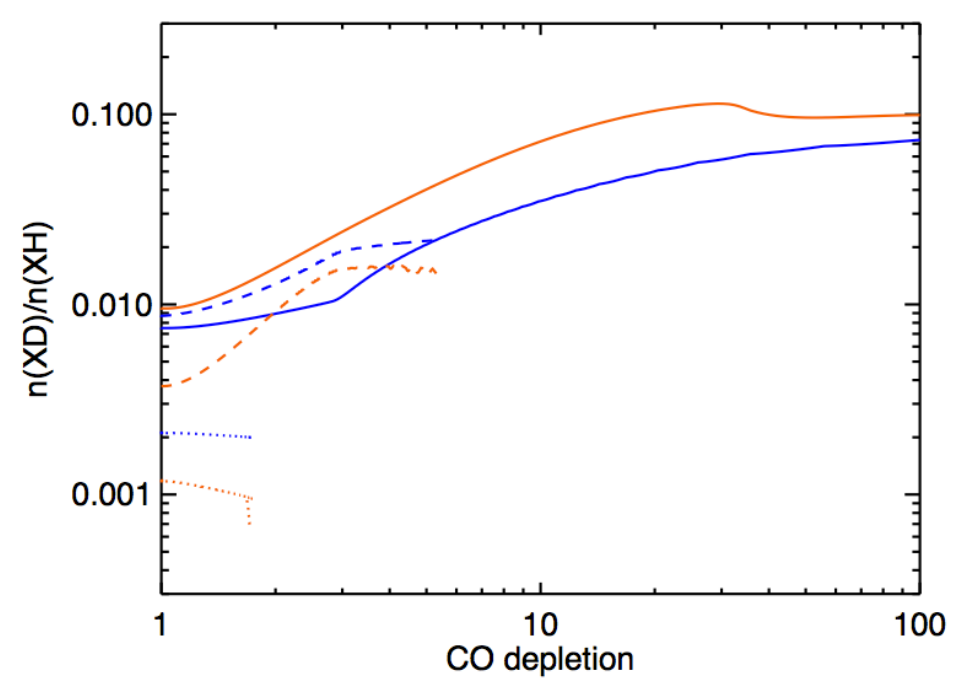

Fig. 19 Theoretical abundances of the $\mathrm{HDO} / \mathrm{H}_{2} \mathrm{O}$ (blue curves) and $\mathrm{D} / \mathrm{H}$ (orange curves) as a function of the $\mathrm{CO}$ depletion, for three different densities and incident visual extinction. Dotted lines: $10^{4} \mathrm{~cm}^{-3}$ and 2 mag; dashed lines: $10^{4} \mathrm{~cm}^{-3}$ and 4 mag; solid lines: $10^{5} \mathrm{~cm}^{-3}$ and 10 mag. Courtesy of (411]).

was very low but also the gas was deprived of $\mathrm{CO}$, 4.1 , or the presence of similar conditions in some zones of the Solar System up to a late stage of the protoplanetary disk phase.

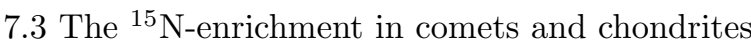

In our Solar System, the ${ }^{15} \mathrm{~N}$ enhancement spans a large range of values. As seen in $3.4 .{ }^{14} \mathrm{~N} /{ }^{15} \mathrm{~N}$ is around 150 in comets, $<300$ in "primitive" material (such as IDPs and carbonaceous chondrites), $<100$ in interplanetary dust particles (IDPs) and carbonaceous chondrites "hotspots", where the largest Dand ${ }^{15} \mathrm{~N}$ fractions have been measured (e.g. [298]), 450 in Jupiter's atmosphere (representative of the protosolar value), 272 on Earth. In pre-stellar cores $(\$ 4)$, current data show a differential ${ }^{15} \mathrm{~N}$-fractionation between amineand nitrile-bearing species, with the largest ${ }^{15} \mathrm{~N}$ enhancement found in the latter (between 70 and 380 in $\mathrm{HCN}$ and HNC; [56]), while no significant enhancement is found in $\mathrm{NH}_{3}$ and $\mathrm{N}_{2} \mathrm{H}^{+}$, both highly D-fractionated. The situation is summarised in Fig. 17. Thus, D- and ${ }^{15} \mathrm{~N}$-fractionations do not go hand in hand in all species within pre-stellar cores, resembling the mixed level of correlation between D- and ${ }^{15} \mathrm{~N}$-enrichments in primitive material of our Solar System. Moreover, the cometary ${ }^{15} \mathrm{~N}$-enhancement has been measured in HCN 
and $\mathrm{CN}$, again suggesting a pre-stellar core origin. It will be interesting to find out if amines will ever experience significant ${ }^{15} \mathrm{~N}$ at all during the pre- and/or proto- stellar phase. Wirström et al. (466) predict large ${ }^{15} \mathrm{~N}$-fractionation in $\mathrm{NH}_{3}$ at late times (>a few million years), so maybe only relatively longlived dense cores will have the chance to have both amines and nitriles highly ${ }^{15} \mathrm{~N}$-fractionated. However, this has all to be tested with observations, which should be extended also to the starless cores found in massive star-forming regions (e.g. 458) to check if environmental conditions affect the fractionation process. As ${ }^{15} \mathrm{~N}$-enhancement has been measured in IOMs and amino acids trapped in carbonaceous chondrites (\$3.4), amines in pre-stellar cores should also be able to experience significant enhancement, if a link between these two extreme stages has to be found ([56]). However, we do not know if further processes within icy mantles or within the "rocks" made out of coagulated icy dust grains, during the proto-Sun stage, would affect the ${ }^{15} \mathrm{~N}$-fractionation in IOM and amino acids found in carbonaceous chondrites. Experiments are needed here.

\section{Concluding remarks}

Our chemical heritage is hidden in the large amount of information obtained by observations of Solar System bodies and star and planet-forming regions, and it needs to be deciphered. In this review, glimpses of links between the present star and planet formation in our Galaxy and the remote past of our Solar System have been given. A summary of these glimpses is given here, together with comments/open questions and suggestions for future developments:

- Water on Earth. The total amount of water on Earth is $\geq 2 \times 10^{-3}$ Earth masses, a small fraction of the total amount of water vapor measured in a pre-stellar core $(\simeq 800$ Earth masses $)$ and in a protoplanetary disk $(\simeq 1.5$ Earth masses), and a negligible factor of the deduced water ice mass in the pre-stellar core and protoplanetary disk (at least three orders of magnitude larger than the water vapor mass). Thus, a large water reservoir was originally available to seed a large number of Solar System bodies, as in fact observed in moons, comets, KBOs and asteroids. Tracing the formation, storage and delivery of water will require more observations as well as a better understanding of the icy-dust coagulation process during the pre-stellar and protoplanetary-disk phases.

- Complex organic molecules, COMs. Organic material in meteorites and IDPs is organized in aromatic and aliphatic compounds, carboxylic acids and amino acids, including those found in all living beings on Earth. PAHs, hydrocarbons and complex organic molecules observed in star-forming regions are a simpler version (building blocks) of the organic material found in Solar System bodies. Thus, interstellar COMs may have contributed to the formation of organic matter during the processing of coagulated icy dust particles once the proto-Sun was born. More experiments are needed 
to confirm this statement. Moreover, COMs observed in comets have abundances relative to methanol more than a factor of ten lower than those measured in hot corinos, possibly suggesting that the hot corino conditions and chemical history may not be representative of our Solar System. It is also possible that the difference arises because of a substantial reprocessing of the protostellar material during the protoplanetary disk phase. Finally, the chemical composition of the envelopes of solar-type protostars in crowded environments populated by massive stars, as suggested in the case of the proto-Sun, has not so far been studied, due to the sensitivity of the available instrumentation. The advent of ALMA should clarify this aspect in the near future.

- D-fractionation in water. The $\mathrm{HDO} / \mathrm{H}_{2} \mathrm{O}$ abundance ratio measured in comets is between 1 and 2 times that measured in our oceans $\left(1.5 \times 10^{-4}\right)$, whereas in cold envelopes and hot corinos a larger spread of this ratio has been found: from $\leq 6 \times 10^{-4}$ to 0.2 . Is the $\mathrm{D}$-fraction in water set at the beginning of the star formation process or is it modified during the various phases of star and planet formation? Or does the water D-enrichment observed in hot corinos probe only the outer layers of the ices, those that sublimate first, while the bulk of the ice, less deuterated as probably inherited in the previous phases, remains frozen and hidden? Is this the ice that we observe in comets? And which process forms water in comets if it is not inherited? Is it surface chemistry, like in pre-stellar objects, or gas-phase chemistry? In order to answer all these questions, we will need to measure the $\mathrm{HDO} / \mathrm{H}_{2} \mathrm{O}$ in different stages of the protostellar evolution and use observations to constrain detailed chemical models, where gas-phase and surface processes are linked, spanning a broad range of physical conditions.

- D-fractionation in other molecules. The D-fractionation is an active process in the cold gas of molecular clouds and becomes one of the dominant chemical processes within pre-stellar cores, in regions where abundant neutral species (mainly $\mathrm{CO}$ and $\mathrm{O}$ ) freeze-out onto dust grains. Here, the increase of the $\mathrm{D} / \mathrm{H}$ elemental ratio in the gas-phase is thought to be responsible for the efficient deuteration of methanol, which happens on the surface of dust grains (as no gas-phase routes are available). Organic molecules such as methanol and formaldehyde observed in star-forming regions (in particular toward low-mass protostellar envelopes) display a D-fraction orders of magnitude higher than that measured in water. This differential D-fractionation of water and organics is also measured in comets, where $\mathrm{DCN} / \mathrm{HCN} \sim 10$ times $\mathrm{HDO} / \mathrm{H}_{2} \mathrm{O}$. Similarly, in the hot spots of carbonaceous chondrites and IDPs, the $\mathrm{D} / \mathrm{H}$ associated with organic radicals reaches values as high as $1 \%$, suggesting, in this case, a direct link with the pre- and proto-stellar phases of the Solar System's formation.

- ${ }^{15} \mathrm{~N}$-fractionation. ${ }^{15} \mathrm{~N}$-enrichments in $\mathrm{HCN}$ and $\mathrm{HNC}$ are measured in comets, with values similar to those observed in pre-stellar cores and Galactic star-forming regions. No significant ${ }^{15} \mathrm{~N}$-fractionation is measured in $\mathrm{NH}_{3}$ and $\mathrm{N}_{2} \mathrm{H}^{+}$, which are, on the other hand, highly deuterated during the pre-stellar core phase. Thus, no significant correlation is expected be- 
tween D- and ${ }^{15} \mathrm{~N}$-fractionated material in Solar System bodies, as in fact measured. More observations of ${ }^{15} \mathrm{~N}$ isotopologues of $\mathrm{NH}_{3}$ and $\mathrm{N}_{2} \mathrm{H}^{+}$in a larger sample of pre-stellar cores (also including massive pre-stellar cores) are needed to look for possible fractionations of these species and to understand if different environmental conditions affect the fractionation process.

To further advance in this field, different communities need to join the effort and work together. In particular, the star formation and Solar System communities should continuously exchange new results and information on new measurements, experiments and theoretical developments. At the same time, laboratory work on molecular spectroscopy to identify the observed lines, on rate coefficients to understand chemical pathways, on surface chemistry and dust coagulation to understand ice formation and ice/dust evolution, as well as calculations of collisional coefficients required for radiative transfer studies are all necessary for a correct interpretation of observations. Finally, theoretical and observational astrophysicists and astrochemists should work together to make sure that, on the one hand, the best physical and dynamical model is used as input for astrochemical modeling and, on the other hand, the best physical parameters derived from the combination of observations, astrochemistry and radiative transfer, are used as input in the physical/dynamical models of star and planet-forming regions. To understand our origins, we cannot work alone!

Acknowledgements We wish to thank our many colleagues for enlightening discussions over the years: L. Bizzocchi, A. Boley, S. Bottinelli, E. Caux, S. Cazaux, C. Codella, A. Crapsi, L. Dore, T. Douglas, F. Fontani, E. Herbst, A. Faure, J. Henshaw, P. Hily-Blant, J. Ilee, I. Jimenez-Serra, T. Hartquist, C. Kahane, E. Keto, B. Lelfoch, P. Myers, J. Pineda, M. Spaans, M. Tafalla, V. Taquet, J. Tan, C. Vastel, M. Walmsley and L. Wiesenfeld. In addition, C.Ceccarelli acknowledges the stimulating discussions with M. Chaussidon, B. Marty and F. Robert on the meteorites. P. Caselli acknowledges the financial support of successive rolling grants awarded by the UK Science and Technology Funding Council. C. Ceccarelli acknowledges the financial support from the Agence Nationale pour la Recherche (ANR), France (project FORCOMS, contracts ANR-08-BLAN-022, and the CNES (Centre National d'Etudes Spatiales).

\section{References}

1. Acke, B., van den Ancker, M.E.: ISO spectroscopy of disks around Herbig Ae/Be stars. A\&A, 426, 151-170 (2004). DOI 10.1051/0004-6361:20040400

2. Adams, F.C.: The Birth Environment of the Solar System. ARA\&A, 48, 47-85 (2010). DOI 10.1146/annurev-astro-081309-130830

3. Adande, G.R., Ziurys, L.M.: Millimeter-wave Observations of $\mathrm{CN}$ and $\mathrm{HNC}$ and Their ${ }^{15} \mathrm{~N}$ Isotopologues: A New Evaluation of the ${ }^{14} \mathrm{~N} /{ }^{15} \mathrm{~N}$ Ratio across the Galaxy. ApJ, 744, 194 (2012). DOI 10.1088/0004-637X/744/2/194

4. Aikawa, Y., Kamuro, D., Sakon, I., Itoh, Y., Terada, H., Noble, J.A., Pontoppidan, K.M., Fraser, H.J., Tamura, M., Kandori, R., Kawamura, A., Ueno, M.: AKARI observations of ice absorption bands towards edge-on young stellar objects. A\&A, 538, A57 (2012). DOI 10.1051/0004-6361/201015999

5. Aikawa, Y., Nomura, H.: Physical and Chemical Structure of Protoplanetary Disks with Grain Growth. ApJ, 642, 1152-1162 (2006). DOI 10.1086/501114

6. Aikawa, Y., Umebayashi, T., Nakano, T., Miyama, S.M.: Evolution of Molecular Abundances in Protoplanetary Disks with Accretion Flow. ApJ, 519, 705-725 (1999). DOI 10.1086/307400 
7. Aikawa, Y., Wakelam, V., Garrod, R.T., Herbst, E.: Molecular Evolution and Star Formation: From Prestellar Cores to Protostellar Cores. ApJ, 674, 984-996 (2008). DOI $10.1086 / 524096$

8. Ale@ARTICLE2006MPSA..41.5327B, author = Busemann, H. and Alexander, C. M. O. and Nittler, L. R. and Zega, T. J. and Stroud, R. M. and Bajt, S. and Cody, G. D. and Yabuta, H., title = "Correlated Analyses of D- and 15N-rich Carbon Grains from CR2 Chondrite EET 92042", journal = Meteoritics and Planetary Science Supplement, year $=2006$, month $=$ sep, volume $=41$, pages $=5327$, adsurl $=$ http://adsabs.harvard.edu/abs/2006Mthe SAO/NASA Astrophysics Data System on, J.: Multiple Origins of Nitrogen Isotopic Anomalies in Meteorites and Comets. ApJ, 722, 1342-1351 (2010). DOI 10.1088/0004-637X/722/2/1342

9. Alexander, C.M.O... Fogel, M., Yabuta, H., Cody, G.D.: The origin and evolution of chondrites recorded in the elemental and isotopic compositions of their macromolecular organic matter. Geochimica et Cosmochimica Acta71, 4380-4403 (2007). DOI 10. 1016/j.gca.2007.06.052

10. Allegre, C.J., Staudacher, T., Sarda, P., Kurz, M.: Constraints on evolution of earth's mantle from rare gas systematics. Nature, 303, 762-766 (1983). DOI 10.1038/303762a0

11. Allen, A., Li, Z.Y., Shu, F.H.: Collapse of Magnetized Singular Isothermal Toroids. II. Rotation and Magnetic Braking. ApJ, 599, 363-379 (2003). DOI 10.1086/379243

12. Andrews, S.M., Williams, J.P.: High-Resolution Submillimeter Constraints on Circumstellar Disk Structure. ApJ, 659, 705-728 (2007). DOI 10.1086/511741

13. Andrews, S.M., Wilner, D.J., Espaillat, C., Hughes, A.M., Dullemond, C.P., McClure, M.K., Qi, C., Brown, J.M.: Resolved Images of Large Cavities in Protoplanetary Transition Disks. ApJ, 732, 42 (2011). DOI 10.1088/0004-637X/732/1/42

14. Andrews, S.M., Wilner, D.J., Hughes, A.M., Qi, C., Dullemond, C.P.: Protoplanetary Disk Structures in Ophiuchus. ApJ, 700, 1502-1523 (2009). DOI 10.1088/0004-637X/ $700 / 2 / 1502$

15. Arce, H.G., Borkin, M.A., Goodman, A.A., Pineda, J.E., Beaumont, C.N.: A Bubbling Nearby Molecular Cloud: COMPLETE Shells in Perseus. ApJ, 742, 105 (2011). DOI 10.1088/0004-637X/742/2/105

16. Arce, H.G., Santiago-García, J., Jørgensen, J.K., Tafalla, M., Bachiller, R.: Complex Molecules in the L1157 Molecular Outflow. ApJL, 681, L21-L24 (2008). DOI 10.1086/ 590110

17. Aresu, G., Meijerink, R., Kamp, I., Spaans, M., Thi, W.F., Woitke, P.: FUV and Xray irradiated protoplanetary disks: a grid of models II - Gas diagnostic line emission. ArXiv e-prints (2012)

18. Armitage, P.J.: Dynamics of Protoplanetary Disks. ARA\&A, 49, 195-236 (2011). DOI 10.1146/annurev-astro-081710-102521

19. Arpigny, C., Jehin, E., Manfroid, J., Hutsemékers, D., Schulz, R., Stüwe, J.A., Zucconi, J.M., Ilyin, I.: Anomalous Nitrogen Isotope Ratio in Comets. Science 301, 1522-1525 (2003). DOI 10.1126/science.1086711

20. Arquilla, R., Goldsmith, P.F.: A detailed examination of the kinematics of rotating dark clouds. ApJ, 303, 356-374 (1986). DOI 10.1086/164082

21. Awad, Z., Viti, S., Collings, M.P., Williams, D.A.: Warm cores around regions of lowmass star formation. MNRAS, 407, 2511-2518 (2010). DOI 10.1111/j.1365-2966.2010. 17077.x

22. Bachiller, R., Perez Gutierrez, M.: Shock Chemistry in the Young Bipolar Outflow L1157. ApJL, 487, L93 (1997). DOI 10.1086/310877

23. Bacmann, A., Lefloch, B., Ceccarelli, C., Castets, A., Steinacker, J., Loinard, L.: The degree of CO depletion in pre-stellar cores. A\&A, 389, L6-L10 (2002). DOI 10.1051/ 0004-6361:20020652

24. Bacmann, A., Lefloch, B., Ceccarelli, C., Steinacker, J., Castets, A., Loinard, L.: CO Depletion and Deuterium Fractionation in Prestellar Cores. ApJL, 585, L55-L58 (2003). DOI 10.1086/374263

25. Bacmann, A., Taquet, V., Faure, A., Kahane, C., Ceccarelli, C.: Detection of complex organic molecules in a prestellar core: a new challenge for astrochemical models. A\&A, 541, L12 (2012). DOI 10.1051/0004-6361/201219207

26. Barucci, M.A., Belskaya, I.N., Fulchignoni, M., Birlan, M.: Taxonomy of Centaurs and Trans-Neptunian Objects. AJ, 130, 1291-1298 (2005). DOI 10.1086/431957 
27. Barucci, M.A., Dotto, E., Levasseur-Regourd, A.C.: Space missions to small bodies: asteroids and cometary nuclei. A\&ARev, 19, 48 (2011). DOI 10.1007/s00159-011-0048-2

28. Basu, S., Mouschovias, T.C.: Magnetic braking, ambipolar diffusion, and the formation of cloud cores and protostars. 1: Axisymmetric solutions. ApJ, 432, 720-741 (1994). DOI 10.1086/174611

29. Beckwith, S.V.W., Sargent, A.I., Chini, R.S., Guesten, R.: A survey for circumstellar disks around young stellar objects. AJ, 99, 924-945 (1990). DOI 10.1086/115385

30. Benedettini, M., Busquet, G., Lefloch, B., Codella, C., Cabrit, S., Ceccarelli, C., Giannini, T., Nisini, B., Vasta, M., Cernicharo, J., Lorenzani, A., di Giorgio, A.M.: The CHESS survey of the L1157-B1 shock: the dissociative jet shock as revealed by Herschel-PACS. A\&A, 539, L3 (2012). DOI 10.1051/0004-6361/201118732

31. Benedettini, M., Giannini, T., Nisini, B., Tommasi, E., Lorenzetti, D., Di Giorgio, A.M., Saraceno, P., Smith, H.A., White, G.J.: The ISO spectroscopic view of the HH 24-26 region. A\&A, 359, 148-158 (2000)

32. Benedettini, M., Viti, S., Giannini, T., Nisini, B., Goldsmith, P.F., Saraceno, P.: Comparing SWAS and ISO observations of water in outflows. A\&A, 395, 657-662 (2002). DOI 10.1051/0004-6361:20021303

33. Bennett, C.J., Jamieson, C.S., Osamura, Y., Kaiser, R.I.: Laboratory Studies on the Irradiation of Methane in Interstellar, Cometary, and Solar System Ices. ApJ, 653, 792-811 (2006). DOI 10.1086/508561

34. Bennett, C.J., Kaiser, R.I.: On the Formation of Glycolaldehyde $\left(\mathrm{HCOCH}_{2} \mathrm{OH}\right)$ and Methyl Formate $\left(\mathrm{HCOOCH}_{3}\right)$ in Interstellar Ice Analogs. ApJ, 661, 899-909 (2007). DOI 10.1086/516745

35. Bergin, E., Calvet, N., D’Alessio, P., Herczeg, G.J.: The Effects of UV Continuum and Ly $\alpha$ Radiation on the Chemical Equilibrium of T Tauri Disks. ApJL, 591, L159-L162 (2003). DOI 10.1086/377148

36. Bergin, E., Calvet, N., Sitko, M.L., Abgrall, H., D’Alessio, P., Herczeg, G.J., Roueff, E., Qi, C., Lynch, D.K., Russell, R.W., Brafford, S.M., Perry, R.B.: A New Probe of the Planet-forming Region in T Tauri Disks. ApJL, 614, L133-L136 (2004). DOI $10.1086 / 425865$

37. Bergin, E.A., Aikawa, Y., Blake, G.A., van Dishoeck, E.F.: The Chemical Evolution of Protoplanetary Disks. Protostars and Planets V pp. 751-766 (2007)

38. Bergin, E.A., Alves, J., Huard, T., Lada, C.J.: $\mathrm{N}_{2} \mathrm{H}^{+}$and $\mathrm{C}^{18} \mathrm{O}$ Depletion in a Cold Dark Cloud. ApJL, 570, L101-L104 (2002). DOI 10.1086/340950

39. Bergin, E.A., Plume, R., Williams, J.P., Myers, P.C.: The Ionization Fraction in Dense Molecular Gas. II. Massive Cores. ApJ, 512, 724-739 (1999). DOI 10.1086/306791

40. Bergin, E.A., Tafalla, M.: Cold Dark Clouds: The Initial Conditions for Star Formation. ARA\&A, 45, 339-396 (2007). DOI 10.1146/annurev.astro.45.071206.100404

41. Bergman, P., Parise, B., Liseau, R., Larsson, B.: Deuterated formaldehyde in $\rho$ Ophiuchi A. A\&A, 527, A39 (2011). DOI 10.1051/0004-6361/201015012

42. Bernstein, M.P., Dworkin, J.P., Sandford, S.A., Cooper, G.W., Allamandola, L.J.: Racemic amino acids from the ultraviolet photolysis of interstellar ice analogues. Nature, 416, 401-403 (2002)

43. Biver, N., Bockelée-Morvan, D., Crovisier, J., Colom, P., Henry, F., Moreno, R., Paubert, G., Despois, D., Lis, D.C.: Chemical Composition Diversity Among 24 Comets Observed At Radio Wavelengths. Earth Moon and Planets 90, 323-333 (2002)

44. Bizzocchi, L., Caselli, P., Dore, L.: Detection of $\mathrm{N}^{15} \mathrm{NH}^{+}$in L1544. A\&A, 510, L5 (2010). DOI 10.1051/0004-6361/200913835

45. Bjerkeli, P., Liseau, R., Larsson, B., Rydbeck, G., Nisini, B., Tafalla, M., Antoniucci S., Benedettini, M., Bergman, P., Cabrit, S., Giannini, T., Melnick, G., Neufeld, D., Santangelo, G., van Dishoeck, E.F.: $\mathrm{H}_{2} \mathrm{O}$ line mapping at high spatial and spectral resolution. Herschel observations of the VLA 1623 outflow. A\&A, 546, A29 (2012). DOI 10.1051/0004-6361/201219776

46. Bjerkeli, P., Liseau, R., Nisini, B., Tafalla, M., Benedettini, M., Bergman, P., Dionatos, O., Giannini, T., Herczeg, G., Justtanont, K., Larsson, B., McOey, C., Olberg, M., Olofsson, A.O.H.: Herschel observations of the Herbig-Haro objects HH 52-54. A\&A, 533, A80 (2011). DOI 10.1051/0004-6361/201116846 
47. Bjerkeli, P., Liseau, R., Olberg, M., Falgarone, E., Frisk, U., Hjalmarson, Å., Klotz, A., Larsson, B., Olofsson, A.O.H., Olofsson, G., Ristorcelli, I., Sandqvist, A.: Odin observations of water in molecular outflows and shocks. A\&A, 507, 1455-1466 (2009). DOI 10.1051/0004-6361/200912064

48. Blake, G.A., Sutton, E.C., Masson, C.R., Phillips, T.G.: Molecular abundances in OMC-1 - The chemical composition of interstellar molecular clouds and the influence of massive star formation. ApJ, 315, 621-645 (1987). DOI 10.1086/165165

49. Bockelée-Morvan, D.: An Overview of Comet Composition. In: IAU Symposium, IAU Symposium, vol. 280, pp. 261-274 (2011). DOI 10.1017/S1743921311025038

50. Bockelée-Morvan, D., Biver, N., Jehin, E., Cochran, A.L., Wiesemeyer, H., Manfroid, J., Hutsemékers, D., Arpigny, C., Boissier, J., Cochran, W., Colom, P., Crovisier, J., Milutinovic, N., Moreno, R., Prochaska, J.X., Ramirez, I., Schulz, R., Zucconi, J.M.: Large Excess of Heavy Nitrogen in Both Hydrogen Cyanide and Cyanogen from Comet 17P/Holmes. ApJL, 679, L49-L52 (2008). DOI 10.1086/588781

51. Bockelée-Morvan, D., Biver, N., Swinyard, B., de Val-Borro, M., Crovisier, J., Hartogh, P., Lis, D.C., Moreno, R., Szutowicz, S., Lellouch, E., Emprechtinger, M., Blake, G.A., Courtin, R., Jarchow, C., Kidger, M., Küppers, M., Rengel, M., Davis, G.R., Fulton, T., Naylor, D., Sidher, S., Walker, H.: Herschel measurements of the $\mathrm{D} / \mathrm{H}$ and ${ }^{16} \mathrm{O} /{ }^{18} \mathrm{O}$ ratios in water in the Oort-cloud comet C/2009 P1 (Garradd). A\&A, 544, L15 (2012). DOI 10.1051/0004-6361/201219744

52. Bockelee-Morvan, D., Gautier, D., Lis, D.C., Young, K., Keene, J., Phillips, T., Owen, T., Crovisier, J., Goldsmith, P.F., Bergin, E.A., Despois, D., Wootten, A.: Deuterated Water in Comet C/1996 B2 (Hyakutake) and Its Implications for the Origin of Comets. Icarus, 133, 147-162 (1998). DOI 10.1006/icar.1998.5916

53. Boduch, P., Domaracka, A., Fulvio, D., Langlinay, T., Lv, X.Y., Palumbo, M.E. Rothard, H., Strazzulla, G.: Chemistry induced by energetic ions in water ice mixed with molecular nitrogen and oxygen. A\&A, 544, A30 (2012). DOI 10.1051/0004-6361/ 201219365

54. Boley, A.C.: The Two Modes of Gas Giant Planet Formation. ApJL, 695, L53-L57 (2009). DOI 10.1088/0004-637X/695/1/L53

55. Boley, A.C., Durisen, R.H.: Gravitational Instabilities, Chondrule Formation, and the FU Orionis Phenomenon. ApJ, 685, 1193-1209 (2008). DOI 10.1086/591013

56. Bonal, L., Hily-Blant, P., Faure, A., Quirico, E.: Highly Variable 15N-Enrichments in Solar System Reflect Different Routes of Interstellar N Isotopic Fractionation. Meteoritics and Planetary Science Supplement 75, 5226 (2012)

57. Bonal, L., Huss, G.R., Krot, A.N., Nagashima, K., Ishii, H.A., Bradley, J.P.: Highly ${ }^{15} \mathrm{~N}$-enriched chondritic clasts in the $\mathrm{CB} / \mathrm{CH}$-like meteorite Isheyevo. Geochimica et Cosmochimica Acta74, 6590-6609 (2010). DOI 10.1016/j.gca.2010.08.017

58. Bonal, L., Huss, G.R., Nagashima, K., Krot, A.N.: Hydrogen Isotopic Composition of $15 \mathrm{~N}$-rich Clasts in the $\mathrm{CB} / \mathrm{CH}-$ like Chondrite Isheyevo. Meteoritics and Planetary Science Supplement 72, 5178 (2009)

59. Boss, A.P.: Giant planet formation by gravitational instability. Science 276, 1836-1839 (1997). DOI 10.1126/science.276.5320.1836

60. Bottinelli, S., Ceccarelli, C., Lefloch, B., Williams, J.P., Castets, A., Caux, E., Cazaux, S., Maret, S., Parise, B., Tielens, A.G.G.M.: Complex Molecules in the Hot Core of the Low-Mass Protostar NGC 1333 IRAS 4A. ApJ, 615, 354-358 (2004). DOI 10.1086/ 423952

61. Bottinelli, S., Ceccarelli, C., Neri, R., Williams, J.P., Caux, E., Cazaux, S., Lefloch, B., Maret, S., Tielens, A.G.G.M.: Near-Arcsecond Resolution Observations of the Hot Corino of the Solar-Type Protostar IRAS 16293-2422. ApJL, 617, L69-L72 (2004). DOI 10.1086/426964

62. Bottinelli, S., Ceccarelli, C., Williams, J.P., Lefloch, B.: Hot corinos in NGC 1333IRAS4B and IRAS2A. A\&A, 463, 601-610 (2007). DOI 10.1051/0004-6361:20066242

63. Bouwman, J., Cuppen, H.M., Steglich, M., Allamandola, L.J., Linnartz, H.: Photochemistry of polycyclic aromatic hydrocarbons in cosmic water ice. II. Near UV/VIS spectroscopy and ionization rates. A\&A, 529, A46 (2011). DOI 10.1051/0004-6361/ 201015762 
64. Bouwman, J., Mattioda, A.L., Linnartz, H., Allamandola, L.J.: Photochemistry of polycyclic aromatic hydrocarbons in cosmic water ice. I. Mid-IR spectroscopy and photoproducts. A\&A, 525, A93 (2011). DOI 10.1051/0004-6361/201015059

65. Braiding, C.R., Wardle, M.: The Hall effect in star formation. MNRAS, 422, 261-281 (2012). DOI 10.1111/j.1365-2966.2012.20601.x

66. Brasser, R.: A two-stage formation process for the Oort comet cloud and its implications. A\&A, 492, 251-255 (2008). DOI 10.1051/0004-6361:200810452

67. Broten, N.W., MacLeod, J.M., Avery, L.W., Irvine, W.M., Hoglund, B., Friberg, P. Hjalmarson, A.: The detection of interstellar methylcyanoacetylene. ApJL, 276, L25L29 (1984). DOI 10.1086/184181

68. Brown, D.W., Chandler, C.J., Carlstrom, J.E., Hills, R.E., Lay, O.P., Matthews, B.C., Richer, J.S., Wilson, C.D.: A submillimetre survey for protostellar accretion discs using the JCMT-CSO interferometer. MNRAS, 319, 154-162 (2000). DOI 10.1046/j.1365-8711.2000.03805.x

69. Brown, M.E.: The Compositions of Kuiper Belt Objects. Annual Review of Earth and Planetary Sciences 40, 467-494 (2012). DOI 10.1146/annurev-earth-042711-105352

70. Brown, M.E., Schaller, E.L., Fraser, W.C.: Water Ice in the Kuiper Belt. AJ, 143, 146 (2012). DOI 10.1088/0004-6256/143/6/146

71. Brunetto, R., Borg, J., Dartois, E., Rietmeijer, F.J.M., Grossemy, F., Sandt, C., Le Sergeant D'Hendecourt, L., Rotundi, A., Dumas, P., Djouadi, Z., Jamme, F.: Mid-IR, Far-IR, Raman micro-spectroscopy, and FESEM-EDX study of IDP L2021C5: Clues to its origin. Icarus, 212, 896-910 (2011). DOI 10.1016/j.icarus.2011.01.038

72. Brünken, S., Gupta, H., Gottlieb, C.A., McCarthy, M.C., Thaddeus, P.: Detection of the Carbon Chain Negative Ion $\mathrm{C}_{8} \mathrm{H}^{-}$in TMC-1. ApJL, 664, L43-L46 (2007). DOI 10.1086/520703

73. Butner, H.M., Charnley, S.B., Ceccarelli, C., Rodgers, S.D., Pardo, J.R., Parise, B. Cernicharo, J., Davis, G.R.: Discovery of Interstellar Heavy Water. ApJL, 659, L137L140 (2007). DOI 10.1086/517883

74. Calvet, N., Briceño, C., Hernández, J., Hoyer, S., Hartmann, L., Sicilia-Aguilar, A., Megeath, S.T., D'Alessio, P.: Disk Evolution in the Orion OB1 Association. AJ, 129 935-946 (2005). DOI 10.1086/426910

75. Calvet, N., D’Alessio, P., Watson, D.M., Franco-Hernández, R., Furlan, E., Green, J., Sutter, P.M., Forrest, W.J., Hartmann, L., Uchida, K.I., Keller, L.D., Sargent, B., Najita, J., Herter, T.L., Barry, D.J., Hall, P.: Disks in Transition in the Taurus Population: Spitzer IRS Spectra of GM Aurigae and DM Tauri. ApJL, 630, L185-L188 (2005). DOI 10.1086/491652

76. Cameron, A.G.W., Truran, J.W.: The supernova trigger for formation of the solar system. Icarus, 30, 447-461 (1977). DOI 10.1016/0019-1035(77)90101-4

77. Carr, J.S., Najita, J.R.: Organic Molecules and Water in the Planet Formation Region of Young Circumstellar Disks. Science 319, 1504- (2008). DOI 10.1126/science.1153807

78. Caselli, P., Benson, P.J., Myers, P.C., Tafalla, M.: Dense Cores in Dark Clouds. XIV. $\mathrm{N}_{2} \mathrm{H}^{+}$(1-0) Maps of Dense Cloud Cores. ApJ, 572, 238-263 (2002). DOI 10.1086/ 340195

79. Caselli, P., Keto, E., Bergin, E.A., Tafalla, M., Aikawa, Y., Douglas, T., Pagani, L. Yildiz, U.A., van der Tak, F.F.S., Walmsley, C.M., Codella, C., Nisini, B., Kristensen, L.E., van Dishoeck, E.F.: First detection of water vapor in a pre-stellar core. ArXiv e-prints (2012)

80. Caselli, P., Stantcheva, T., Shalabiea, O., Shematovich, V.I., Herbst, E.: Deuterium fractionation on interstellar grains studied with modified rate equations and a Monte Carlo approach. P\&SS, 50, 1257-1266 (2002). DOI 10.1016/S0032-0633(02)00092-2

81. Caselli, P., van der Tak, F.F.S., Ceccarelli, C., Bacmann, A.: Abundant $\mathrm{H}_{2} \mathrm{D}^{+}$in the pre-stellar core L1544. A\&A, 403, L37-L41 (2003). DOI 10.1051/0004-6361:20030526

82. Caselli, P., Walmsley, C.M., Tafalla, M., Dore, L., Myers, P.C.: CO Depletion in the Starless Cloud Core L1544. ApJL, 523, L165-L169 (1999). DOI 10.1086/312280

83. Caselli, P., Walmsley, C.M., Terzieva, R., Herbst, E.: The Ionization Fraction in Dense Cloud Cores. ApJ, 499, 234 (1998). DOI 10.1086/305624

84. Caselli, P., Walmsley, C.M., Zucconi, A., Tafalla, M., Dore, L., Myers, P.C.: Molecular Ions in L1544. II. The Ionization Degree. ApJ, 565, 344-358 (2002). DOI 10.1086/ 324302 
85. Caux, E., Kahane, C., Castets, A., Coutens, A., Ceccarelli, C., Bacmann, A., Bisschop, S., Bottinelli, S., Comito, C., Helmich, F.P., Lefloch, B., Parise, B., Schilke, P., Tielens, A.G.G.M., van Dishoeck, E., Vastel, C., Wakelam, V., Walters, A.: TIMASSS: the IRAS 16293-2422 millimeter and submillimeter spectral survey. I. Observations, calibration, and analysis of the line kinematics. A\&A, 532, A23 (2011). DOI 10.1051/0004-6361/201015399

86. Cazaux, S., Caselli, P., Spaans, M.: Interstellar Ices as Witnesses of Star Formation: Selective Deuteration of Water and Organic Molecules Unveiled. ApJL, 741, L34 (2011). DOI 10.1088/2041-8205/741/2/L34

87. Cazaux, S., Cobut, V., Marseille, M., Spaans, M., Caselli, P.: Water formation on bare grains: When the chemistry on dust impacts interstellar gas. A\&A, 522, A74 (2010). DOI 10.1051/0004-6361/201014026

88. Cazaux, S., Tielens, A.G.G.M.: Molecular Hydrogen Formation in the Interstellar Medium. ApJL, 575, L29-L32 (2002). DOI 10.1086/342607

89. Cazaux, S., Tielens, A.G.G.M., Ceccarelli, C., Castets, A., Wakelam, V., Caux, E., Parise, B., Teyssier, D.: The Hot Core around the Low-mass Protostar IRAS 162932422: Scoundrels Rule! ApJL, 593, L51-L55 (2003). DOI 10.1086/378038

90. Ceccarelli, C., Caselli, P., Herbst, E., Tielens, A.G.G.M., Caux, E.: Extreme Deuteration and Hot Corinos: The Earliest Chemical Signatures of Low-Mass Star Formation. Protostars and Planets V pp. 47-62 (2007)

91. Ceccarelli, C., Castets, A., Caux, E., Hollenbach, D., Loinard, L., Molinari, S., Tielens, A.G.G.M.: The structure of the collapsing envelope around the low-mass protostar IRAS 16293-2422. A\&A, 355, 1129-1137 (2000)

92. Ceccarelli, C., Castets, A., Loinard, L., Caux, E., Tielens, A.G.G.M.: Detection of doubly deuterated formaldehyde towards the low-luminosity protostar IRAS 162932422. A\&A, 338, L43-L46 (1998)

93. Ceccarelli, C., Dominik, C., Caux, E., Lefloch, B., Caselli, P.: Discovery of Deuterated Water in a Young Protoplanetary Disk. ApJL, 631, L81-L84 (2005). DOI 10.1086/ 497028

94. Ceccarelli, C., Dominik, C., Lefloch, B., Caselli, P., Caux, E.: Detection of $\mathrm{H}_{2} \mathrm{D}^{+}$: Measuring the Midplane Degree of Ionization in the Disks of DM Tauri and TW Hydrae. ApJL, 607, L51-L54 (2004). DOI 10.1086/421461

95. Ceccarelli, C., Hollenbach, D.J., Tielens, A.G.G.M.: Far-Infrared Line Emission from Collapsing Protostellar Envelopes. ApJ, 471, 400 (1996). DOI 10.1086/177978

96. Ceccarelli, C., Loinard, L., Castets, A., Tielens, A.G.G.M., Caux, E.: The hot core of the solar-type protostar IRAS 16293-2422: H_2CO emission. A\&A, 357, L9-L12 (2000)

97. Chandler, C.J., Koerner, D.W., Sargent, A.I., Wood, D.O.S.: Dust Emission from Protostars: The Disk and Envelope of HH 24 MMS. ApJL, 449, L139 (1995). DOI $10.1086 / 309644$

98. Chapillon, E., Dutrey, A., Guilloteau, S., Piétu, V., Wakelam, V., Hersant, F., Gueth, F., Henning, T., Launhardt, R., Schreyer, K., Semenov, D.: Chemistry in Disks. VII. First Detection of $\mathrm{HC}_{3} \mathrm{~N}$ in Protoplanetary Disks. ApJ, 756, 58 (2012). DOI 10.1088/ 0004-637X/756/1/58

99. Chapillon, E., Guilloteau, S., Dutrey, A., Piétu, V., Guélin, M.: Chemistry in disks. VI. CN and HCN in protoplanetary disks. A\&A, 537, A60 (2012). DOI 10.1051/ 0004-6361/201116762

100. Charnley, S.B., Tielens, A.G.G.M., Millar, T.J.: On the molecular complexity of the hot cores in Orion A - Grain surface chemistry as 'The last refuge of the scoundrel'. ApJL, 399, L71-L74 (1992). DOI 10.1086/186609

101. Charnley, S.B., Tielens, A.G.G.M., Rodgers, S.D.: Deuterated Methanol in the Orion Compact Ridge. ApJL, 482, L203 (1997). DOI 10.1086/310697

102. Chaussidon, M., Srinivasan, G.: New Constraints on the Origin of Short-Lived 10Be in the Early Solar System. Meteoritics and Planetary Science Supplement 75, 5192 (2012)

103. Chiang, H.F., Looney, L.W., Tobin, J.J.: The Envelope and Embedded Disk around the Class 0 Protostar L1157-mm: Dual-wavelength Interferometric Observations and Modeling. ApJ, 756, 168 (2012). DOI 10.1088/0004-637X/756/2/168 
104. Chiar, J.E., Pendleton, Y.J., Allamandola, L.J., Boogert, A.C.A., Ennico, K., Greene, T.P., Geballe, T.R., Keane, J.V., Lada, C.J., Mason, R.E., Roellig, T.L., Sandford, S.A., Tielens, A.G.G.M., Werner, M.W., Whittet, D.C.B., Decin, L., Eriksson, K.: Ices in the Quiescent IC 5146 Dense Cloud. ApJ, 731, 9 (2011). DOI 10.1088/0004-637X/ $731 / 1 / 9$

105. Choi, M., Tatematsu, K., Park, G., Kang, M.: Ammonia Imaging of the Disks in the NGC 1333 IRAS 4A Protobinary System. ApJL, 667, L183-L186 (2007). DOI $10.1086 / 522116$

106. Cieza, L.A., Mathews, G.S., Williams, J.P., Ménard, F.C., Kraus, A.L., Schreiber, M.R., Romero, G.A., Orellana, M., Ireland, M.J.: Submillimeter Array Observations of the RX J1633.9-2442 Transition Disk: Evidence for Multiple Planets in the Making. ApJ, 752, 75 (2012). DOI 10.1088/0004-637X/752/1/75

107. Codella, C., Benedettini, M., Beltrán, M.T., Gueth, F., Viti, S., Bachiller, R., Tafalla, M., Cabrit, S., Fuente, A., Lefloch, B.: Methyl cyanide as tracer of bow shocks in L1157-B1. A\&A, 507, L25-L28 (2009). DOI 10.1051/0004-6361/200913340

108. Codella, C., Ceccarelli, C., Bottinelli, S., Salez, M., Viti, S., Lefloch, B., Cabrit, S., Caux, E., Faure, A., Vasta, M., Wiesenfeld, L.: First Detection of Hydrogen Chloride Toward Protostellar Shocks. ApJ, 744, 164 (2012). DOI 10.1088/0004-637X/744/2/ 164

109. Codella, C., Ceccarelli, C., Lefloch, B., Fontani, F., Busquet, G., Caselli, P., Kahane, C., Lis, D., Taquet, V., Vasta, M., Viti, S., Wiesenfeld, L.: The Herschel and IRAM CHESS Spectral Surveys of the Protostellar Shock L1157-B1: Fossil Deuteration. ApJL, 757, L9 (2012). DOI 10.1088/2041-8205/757/1/L9

110. Codella, C., Lefloch, B., Ceccarelli, C., Cernicharo, J., Caux, E., Lorenzani, A., Viti, S., Hily-Blant, P., Parise, B., Maret, S., Nisini, B., Caselli, P., Cabrit, S., Pagani, L., Benedettini, M., Boogert, A., Gueth, F., Melnick, G., Neufeld, D., Pacheco, S., Salez, M., Schuster, K., Bacmann, A., Baudry, A., Bell, T., Bergin, E.A., Blake, G., Bottinelli, S., Castets, A., Comito, C., Coutens, A., Crimier, N., Dominik, C., Demyk, K., Encrenaz, P., Falgarone, E., Fuente, A., Gerin, M., Goldsmith, P., Helmich, F., Hennebelle, P., Henning, T., Herbst, E., Jacq, T., Kahane, C., Kama, M., Klotz, A., Langer, W., Lis, D., Lord, S., Pearson, J., Phillips, T., Saraceno, P., Schilke, P., Tielens, X., van der Tak, F., van der Wiel, M., Vastel, C., Wakelam, V., Walters, A., Wyrowski, F., Yorke, H., Borys, C., Delorme, Y., Kramer, C., Larsson, B., Mehdi, I., Ossenkopf, V., Stutzki, J.: The CHESS spectral survey of star forming regions: Peering into the protostellar shock L1157-B1. I. Shock chemical complexity. A\&A, 518, L112 (2010). DOI 10.1051/0004-6361/201014582

111. Cordiner, M.A., Charnley, S.B., Wirström, E.S., Smith, R.G.: Organic Chemistry of Low-mass Star-forming Cores. I. $7 \mathrm{~mm}$ Spectroscopy of Chamaeleon MMS1. ApJ, 744, 131 (2012). DOI 10.1088/0004-637X/744/2/131

112. Cortes, S.R., Meyer, M.R., Carpenter, J.M., Pascucci, I., Schneider, G., Wong, T., Hines, D.C.: Grain Growth and Global Structure of the Protoplanetary Disk Associated with the Mature Classical T Tauri Star, PDS 66. ApJ, 697, 1305-1315 (2009). DOI 10.1088/0004-637X/697/2/1305

113. Coutens, A., Vastel, C., Caux, E., Ceccarelli, C., Bottinelli, S., Wiesenfeld, L., Faure, A., Scribano, Y., Kahane, C.: A study of deuterated water in the low-mass protostar IRAS 16293-2422. A\&A, 539, A132 (2012). DOI 10.1051/0004-6361/201117627

114. Crapsi, A., Caselli, P., Walmsley, C.M., Myers, P.C., Tafalla, M., Lee, C.W., Bourke, T.L.: Probing the Evolutionary Status of Starless Cores through $\mathrm{N}_{2} \mathrm{H}^{+}$and $\mathrm{N}_{2} \mathrm{D}^{+}$ Observations. ApJ, 619, 379-406 (2005). DOI 10.1086/426472

115. Crapsi, A., Caselli, P., Walmsley, M.C., Tafalla, M.: Observing the gas temperature drop in the high-density nucleus of L 1544. A\&A, 470, 221-230 (2007). DOI 10.1051/ 0004-6361:20077613

116. Crimier, N., Ceccarelli, C., Lefloch, B., Faure, A.: Physical structure and water line spectrum predictions of the intermediate mass protostar OMC2-FIR4. A\&A, 506, 1229-1241 (2009). DOI 10.1051/0004-6361/200911651

117. Crimier, N., Ceccarelli, C., Maret, S., Bottinelli, S., Caux, E., Kahane, C., Lis, D.C., Olofsson, J.: The solar type protostar IRAS16293-2422: new constraints on the physical structure. A\&A, 519, A65 (2010). DOI 10.1051/0004-6361/200913112 
118. Crovisier, J., Biver, N., Bockelée-Morvan, D., Boissier, J., Colom, P., Lis, D.C.: The Chemical Diversity of Comets: Synergies Between Space Exploration and Groundbased Radio Observations. Earth Moon and Planets 105, 267-272 (2009). DOI $10.1007 / \mathrm{s} 11038-009-9293-\mathrm{z}$

119. Cuppen, H.M., Herbst, E.: Monte Carlo simulations of $\mathrm{H}_{2}$ formation on grains of varying surface roughness. MNRAS, 361, 565-576 (2005). DOI 10.1111/j.1365-2966. 2005.09189.x

120. Cuppen, H.M., van Dishoeck, E.F., Herbst, E., Tielens, A.G.G.M.: Microscopic simulation of methanol and formaldehyde ice formation in cold dense cores. A\&A, 508 275-287 (2009). DOI 10.1051/0004-6361/200913119

121. Czaja, A.D.: Early Earth: Microbes and the rise of oxygen. Nature Geoscience 3 522-523 (2010). DOI 10.1038/ngeo929

122. D'Alessio, P., Calvet, N., Hartmann, L.: Accretion Disks around Young Objects. III. Grain Growth. ApJ, 553, 321-334 (2001). DOI 10.1086/320655

123. D'Alessio, P., Calvet, N., Hartmann, L., Franco-Hernández, R., Servín, H.: Effects of Dust Growth and Settling in T Tauri Disks. ApJ, 638, 314-335 (2006). DOI $10.1086 / 498861$

124. D'Alessio, P., Calvet, N., Hartmann, L., Lizano, S., Cantó, J.: Accretion Disks around Young Objects. II. Tests of Well-mixed Models with ISM Dust. ApJ, 527, 893-909 (1999). DOI 10.1086/308103

125. Dalgarno, A., Lepp, S.: Deuterium fractionation mechanisms in interstellar clouds. ApJL, 287, L47-L50 (1984). DOI 10.1086/184395

126. Dauphas, N.: The dual origin of the terrestrial atmosphere. Icarus, 165, 326-339 (2003). DOI 10.1016/S0019-1035(03)00198-2

127. Dauphas, N., Chaussidon, M.: A Perspective from Extinct Radionuclides on a Young Stellar Object: The Sun and Its Accretion Disk. Annual Review of Earth and Planetary Sciences 39, 351-386 (2011). DOI 10.1146/annurev-earth-040610-133428

128. Dauphas, N., Robert, F., Marty, B.: The Late Asteroidal and Cometary Bombardment of Earth as Recorded in Water Deuterium to Protium Ratio. Icarus, 148, 508-512 (2000). DOI 10.1006/icar.2000.6489

129. Delsemme, A.H.: Cometary origin of carbon and water on the terrestrial planets. Advances in Space Research 12, 5-12 (1992). DOI 10.1016/0273-1177(92)90147-P

130. D'Hendecourt, L.B., Allamandola, L.J., Baas, F., Greenberg, J.M.: Interstellar grain explosions - Molecule cycling between gas and dust. A\&A, 109, L12-L14 (1982)

131. Dominik, C., Blum, J., Cuzzi, J.N., Wurm, G.: Growth of Dust as the Initial Step Toward Planet Formation. Protostars and Planets V pp. 783-800 (2007)

132. Dones, L., Weissman, P.R., Levison, H.F., Duncan, M.J.: Oort cloud formation and dynamics, pp. 153-174 (2004)

133. Doty, S.D., Neufeld, D.A.: Models for Dense Molecular Cloud Cores. ApJ, 489, 122 (1997). DOI 10.1086/304764

134. Dubernet, M.L., Cernicharo, J., Daniel, F., Debray, B., Faure, A., Feautrier, N., Flower D., Grosjean, A., Roueff, E., Spielfiedel, A., Stoecklin, T., Valiron, P.: Ro-vibrational Collisional Excitation Database: BASECOL - http://www.obspm.fr/basecol. In: F. Combes, D. Barret, T. Contini, F. Meynadier, L. Pagani (eds.) SF2A-2004: Semaine de l'Astrophysique Francaise, p. 525 (2004)

135. Duchêne, G., Ménard, F., Stapelfeldt, K., Duvert, G.: A layered edge-on circumstellar disk around HK Tau B. A\&A, 400, 559-565 (2003). DOI 10.1051/0004-6361:20021906

136. Dulieu, F., Amiaud, L., Congiu, E., Fillion, J.H., Matar, E., Momeni, A., Pirronello, V. Lemaire, J.L.: Experimental evidence for water formation on interstellar dust grains by hydrogen and oxygen atoms. A\&A, 512, A30 (2010). DOI 10.1051/0004-6361/ 200912079

137. Dullemond, C.P., Henning, T., Visser, R., Geers, V.C., van Dishoeck, E.F., Pontoppidan, K.M.: Dust sedimentation in protoplanetary disks with polycyclic aromatic hydrocarbons. A\&A, 473, 457-466 (2007). DOI 10.1051/0004-6361:20077581

138. Dullemond, C.P., Hollenbach, D., Kamp, I., D'Alessio, P.: Models of the Structure and Evolution of Protoplanetary Disks. Protostars and Planets V pp. 555-572 (2007)

139. Dullemond, C.P., Monnier, J.D.: The Inner Regions of Protoplanetary Disks. ARA\&A, 48, 205-239 (2010). DOI 10.1146/annurev-astro-081309-130932 
140. Durisen, R.H., Boss, A.P., Mayer, L., Nelson, A.F., Quinn, T., Rice, W.K.M.: Gravitational Instabilities in Gaseous Protoplanetary Disks and Implications for Giant Planet Formation. Protostars and Planets V pp. 607-622 (2007)

141. Dutrey, A., Guilloteau, S., Duvert, G., Prato, L., Simon, M., Schuster, K., Menard, F.: Dust and gas distribution around $\mathrm{T}$ Tauri stars in Taurus-Auriga. I. Interferometric $2.7 \mathrm{~mm}$ continuum and ${ }^{\wedge} 13^{\wedge} \mathrm{CO} \mathrm{J}=1-0$ observations. A\&A, 309, 493-504 (1996)

142. Dutrey, A., Guilloteau, S., Guelin, M.: Chemistry of protosolar-like nebulae: The molecular content of the DM Tau and GG Tau disks. A\&A, 317, L55-L58 (1997)

143. Dutrey, A., Guilloteau, S., Ho, P.: Interferometric Spectroimaging of Molecular Gas in Protoplanetary Disks. Protostars and Planets V pp. 495-506 (2007)

144. Dutrey, A., Henning, T., Guilloteau, S., Semenov, D., Piétu, V., Schreyer, K., Bacmann, A., Launhardt, R., Pety, J., Gueth, F.: Chemistry in disks. I. Deep search for $\mathrm{N}_{2} \mathrm{H}^{+}$in the protoplanetary disks around LkCa 15, MWC 480, and DM Tauri. A\&A, 464, 615-623 (2007). DOI 10.1051/0004-6361:20065385

145. Dutrey, A., Wakelam, V., Boehler, Y., Guilloteau, S., Hersant, F., Semenov, D., Chapillon, E., Henning, T., Piétu, V., Launhardt, R., Gueth, F., Schreyer, K.: Chemistry in disks. V. Sulfur-bearing molecules in the protoplanetary disks surrounding LkCa15, MWC480, DM Tauri, and GO Tauri. A\&A, 535, A104 (2011). DOI 10.1051/0004-6361/201116931

146. Elsila, J.E., Glavin, D.P., Dworkin, J.P.: Cometary glycine detected in samples returned by Stardust. Meteoritics and Planetary Science 44, 1323-1330 (2009). DOI 10.1111/j.1945-5100.2009.tb01224.x

147. Enoch, M.L., Corder, S., Duchêne, G., Bock, D.C., Bolatto, A.D., Culverhouse, T.L., Kwon, W., Lamb, J.W., Leitch, E.M., Marrone, D.P., Muchovej, S.J., Pérez, L.M., Scott, S.L., Teuben, P.J., Wright, M.C.H., Zauderer, B.A.: Disk and Envelope Structure in Class 0 Protostars. II. High-resolution Millimeter Mapping of the Serpens Sample. ApJS, 195, 21 (2011). DOI 10.1088/0067-0049/195/2/21

148. Evans II, N.J., Rawlings, J.M.C., Shirley, Y.L., Mundy, L.G.: Tracing the Mass during Low-Mass Star Formation. II. Modeling the Submillimeter Emission from Preprotostellar Cores. ApJ, 557, 193-208 (2001). DOI 10.1086/321639

149. Fedele, D., Bruderer, S., van Dishoeck, E.F., Herczeg, G.J., Evans, N.J., Bouwman, J., Henning, T., Green, J.: Warm $\mathrm{H}_{2} \mathrm{O}$ and $\mathrm{OH}$ in the disk around the Herbig star HD 163296. A\&A, 544, L9 (2012). DOI 10.1051/0004-6361/201219615

150. Fedele, D., van den Ancker, M.E., Acke, B., van der Plas, G., van Boekel, R., Wittkowski, M., Henning, T., Bouwman, J., Meeus, G., Rafanelli, P.: The structure of the protoplanetary disk surrounding three young intermediate mass stars. II. Spatially resolved dust and gas distribution. A\&A, 491, 809-820 (2008). DOI 10.1051/0004-6361:200810126

151. Feigelson, E.D., Montmerle, T.: High-Energy Processes in Young Stellar Objects. ARA\&A, 37, 363-408 (1999). DOI 10.1146/annurev.astro.37.1.363

152. Fisher, D.E.: Implications of terrestrial Ar-40/Ar-36 for atmospheric and mantle evolutionary models. Physics of the Earth and Planetary Interiors 29, 242-251 (1982). DOI 10.1016/0031-9201(82)90015-2

153. Flower, D.R., Pineau Des Forêts, G., Walmsley, C.M.: The importance of the ortho:para $\mathrm{H}_{2}$ ratio for the deuteration of molecules during pre-protostellar collapse. A\&A, 449, 621-629 (2006). DOI 10.1051/0004-6361:20054246

154. Fogel, J.K.J., Bethell, T.J., Bergin, E.A., Calvet, N., Semenov, D.: Chemistry of a Protoplanetary Disk with Grain Settling and Ly $\alpha$ Radiation. ApJ, 726, 29 (2011). DOI 10.1088/0004-637X/726/1/29

155. Fouchet, T., Irwin, P.G.J., Parrish, P., Calcutt, S.B., Taylor, F.W., Nixon, C.A., Owen, T.: Search for spatial variation in the jovian ${ }^{15} \mathrm{~N} /{ }^{14} \mathrm{~N}$ ratio from Cassini/CIRS observations. Icarus, 172, 50-58 (2004). DOI 10.1016/j.icarus.2003.11.011

156. France, K., Schindhelm, E., Herczeg, G.J., Brown, A., Abgrall, H., Alexander, R.D. Bergin, E.A., Brown, J.M., Linsky, J.L., Roueff, E., Yang, H.: A Hubble Space Telescope Survey of $\mathrm{H}_{2}$ Emission in the Circumstellar Environments of Young Stars. ApJ, 756, 171 (2012). DOI 10.1088/0004-637X/756/2/171

157. Franklin, J., Snell, R.L., Kaufman, M.J., Melnick, G.J., Neufeld, D.A., Hollenbach, D.J., Bergin, E.A.: SWAS Observations of Water in Molecular Outflows. ApJ, 674, 1015-1031 (2008). DOI 10.1086/524924 
158. Frau, P., Galli, D., Girart, J.M.: Comparing star formation models with interferometric observations of the protostar NGC 1333 IRAS 4A. I. Magnetohydrodynamic collapse models. A\&A, 535, A44 (2011). DOI 10.1051/0004-6361/201117813

159. Friberg, P., Hjalmarson, A., Madden, S.C., Irvine, W.M.: Methanol in dark clouds. A\&A, 195, 281-289 (1988)

160. Friesen, R.K., Di Francesco, J., Shimajiri, Y., Takakuwa, S.: The Initial Conditions of Clustered Star Formation. II. $\mathrm{N}_{2} \mathrm{H}^{+}$Observations of the Ophiuchus B Core. ApJ, 708, 1002-1024 (2010). DOI 10.1088/0004-637X/708/2/1002

161. Fuchs, G.W., Cuppen, H.M., Ioppolo, S., Romanzin, C., Bisschop, S.E., Andersson, S., van Dishoeck, E.F., Linnartz, H.: Hydrogenation reactions in interstellar CO ice analogues. A combined experimental/theoretical approach. A\&A, 505, 629-639 (2009). DOI 10.1051/0004-6361/200810784

162. Fuente, A., Cernicharo, J., Agúndez, M., Berné, O., Goicoechea, J.R., Alonso-Albi, T., Marcelino, N.: Molecular content of the circumstellar disk in AB Aurigae. First detection of SO in a circumstellar disk. A\&A, 524, A19 (2010). DOI 10.1051/0004-6361/ 201014905

163. Furlan, E., Hartmann, L., Calvet, N., D’Alessio, P., Franco-Hernández, R., Forrest, W.J., Watson, D.M., Uchida, K.I., Sargent, B., Green, J.D., Keller, L.D., Herter, T.L.: A Survey and Analysis of Spitzer Infrared Spectrograph Spectra of T Tauri Stars in Taurus. ApJS, 165, 568-605 (2006). DOI 10.1086/505468

164. Furuya, K., Aikawa, Y., Tomida, K., Matsumoto, T., Saigo, K., Tomisaka, K., Hersant, F., Wakelam, V.: Chemistry in the First Hydrostatic Core Stage Adopting ThreeDimensional Radiation Hydrodynamic Simulations. ArXiv e-prints (2012)

165. Galli, D., Shu, F.H.: Collapse of Magnetized Molecular Cloud Cores. I. Semianalytical Solution. ApJ, 417, 220 (1993). DOI 10.1086/173305

166. Galli, D., Shu, F.H.: Collapse of Magnetized Molecular Cloud Cores. II. Numerical Results. ApJ, 417, 243 (1993). DOI 10.1086/173306

167. García Ruiz, J.M., Carnerup, A., Christy, A.G., Welham, N.J., Hyde, S.T.: Morphology: An Ambiguous Indicator of Biogenicity. Astrobiology 2, 353-369 (2002). DOI $10.1089 / 153110702762027925$

168. Garrod, R.T., Herbst, E.: Formation of methyl formate and other organic species in the warm-up phase of hot molecular cores. A\&A, 457, 927-936 (2006). DOI 10.1051/ 0004-6361:20065560

169. Garrod, R.T., Pauly, T.: On the Formation of $\mathrm{CO}_{2}$ and Other Interstellar Ices. ApJ, 735, 15 (2011). DOI 10.1088/0004-637X/735/1/15

170. Garrod, R.T., Vasyunin, A.I., Semenov, D.A., Wiebe, D.S., Henning, T.: A New Modified-Rate Approach For Gas-Grain Chemistry: Comparison with a Unified LargeScale Monte Carlo Simulation. ApJL, 700, L43-L46 (2009). DOI 10.1088/0004-637X/ $700 / 1 / \mathrm{L} 43$

171. Garrod, R.T., Wakelam, V., Herbst, E.: Non-thermal desorption from interstellar dust grains via exothermic surface reactions. A\&A, 467, 1103-1115 (2007). DOI 10.1051/ 0004-6361:20066704

172. Garrod, R.T., Weaver, S.L.W., Herbst, E.: Complex Chemistry in Star-forming Regions: An Expanded Gas-Grain Warm-up Chemical Model. ApJ, 682, 283-302 (2008). DOI 10.1086/588035

173. Geers, V.C., Augereau, J.C., Pontoppidan, K.M., Dullemond, C.P., Visser, R., KesslerSilacci, J.E., Evans II, N.J., van Dishoeck, E.F., Blake, G.A., Boogert, A.C.A., Brown, J.M., Lahuis, F., Merín, B.: C2D Spitzer-IRS spectra of disks around T Tauri stars. II. PAH emission features. A\&A, 459, 545-556 (2006). DOI 10.1051/0004-6361:20064830

174. Geiss, J., Gloeckler, G.: Abundances of Deuterium and Helium-3 in the Protosolar Cloud. Space Science Reviews, 84, 239-250 (1998)

175. Gerakines, P.A., Schutte, W.A., Ehrenfreund, P.: Ultraviolet processing of interstellar ice analogs. I. Pure ices. A\&A, 312, 289-305 (1996)

176. Gerin, M., Marcelino, N., Biver, N., Roueff, E., Coudert, L.H., Elkeurti, M., Lis, D.C., Bockelée-Morvan, D.: Detection of ${ }^{15} \mathrm{NH}\{2\} \mathrm{D}$ in dense cores: a new tool for measuring the ${ }^{14} \mathrm{~N} /{ }^{15} \mathrm{~N}$ ratio in the cold ISM. A\&A, 498, L9-L12 (2009). DOI 10.1051/0004-6361/200911759

177. Girart, J.M., Rao, R., Marrone, D.P.: Magnetic Fields in the Formation of Sun-Like Stars. Science 313, 812-814 (2006). DOI 10.1126/science.1129093 
178. Glassgold, A.E., Najita, J., Igea, J.: X-Ray Ionization of Protoplanetary Disks. ApJ, 480, 344 (1997). DOI 10.1086/303952

179. Goldsmith, P.F.: Molecular Depletion and Thermal Balance in Dark Cloud Cores. ApJ, 557, 736-746 (2001). DOI 10.1086/322255

180. Gomes, R., Levison, H.F., Tsiganis, K., Morbidelli, A.: Origin of the cataclysmic Late Heavy Bombardment period of the terrestrial planets. Nature, 435, 466-469 (2005). DOI 10.1038 /nature03676

181. Goodman, A.A., Benson, P.J., Fuller, G.A., Myers, P.C.: Dense cores in dark clouds. VIII - Velocity gradients. ApJ, 406, 528-547 (1993). DOI 10.1086/172465

182. Gorti, U., Hollenbach, D.: Models of Chemistry, Thermal Balance, and Infrared Spectra from Intermediate-Aged Disks around G and K Stars. ApJ, 613, 424-447 (2004). DOI $10.1086 / 422406$

183. Gorti, U., Hollenbach, D.: Line Emission from Gas in Optically Thick Dust Disks around Young Stars. ApJ, 683, 287-303 (2008). DOI 10.1086/589616

184. Goto, M., Carmona, A., Linz, H., Stecklum, B., Henning, T., Meeus, G., Usuda, T.: Kinematics of Ionized Gas at 0.01 AU of TW Hya. ApJ, 748, 6 (2012). DOI 10.1088/ 0004-637X/748/1/6

185. Gounelle, M., Meibom, A.: The Origin of Short-lived Radionuclides and the Astrophysical Environment of Solar System Formation. ApJ, 680, 781-792 (2008). DOI $10.1086 / 587613$

186. Gratton, R.G., Carretta, E., Bragaglia, A.: Multiple populations in globular clusters. Lessons learned from the Milky Way globular clusters. A\&ARev, 20, 50 (2012). DOI 10.1007/s00159-012-0050-3

187. Gredel, R., Lepp, S., Dalgarno, A., Herbst, E.: Cosmic-ray-induced photodissociation and photoionization rates of interstellar molecules. ApJ, 347, 289-293 (1989). DOI $10.1086 / 168117$

188. Guelin, M., Langer, W.D., Snell, R.L., Wootten, H.A.: Observations of DCO/plus/ The electron abundance in dark clouds. ApJL, 217, L165-L168 (1977). DOI 10.1086/ 182562

189. Guhathakurta, P., Draine, B.T.: Temperature fluctuations in interstellar grains. I Computational method and sublimation of small grains. ApJ, 345, 230-244 (1989). DOI 10.1086/167899

190. Guilloteau, S., Piétu, V., Dutrey, A., Guélin, M.: Deuterated molecules in DM Tauri: $\mathrm{DCO}^{+}$, but no HDO. A\&A, 448, L5-L8 (2006). DOI 10.1051/0004-6361:200600005

191. Güttler, C., Blum, J., Zsom, A., Ormel, C.W., Dullemond, C.P.: The outcome of protoplanetary dust growth: pebbles, boulders, or planetesimals?. I. Mapping the zoo of laboratory collision experiments. A\&A, 513, A56 (2010). DOI 10.1051/0004-6361/ 200912852

192. Habart, E., Boulanger, F., Verstraete, L., Walmsley, C.M., Pineau des Forêts, G.: Some empirical estimates of the $\mathrm{H}_{2}$ formation rate in photon-dominated regions. A\&A, 414, 531-544 (2004). DOI 10.1051/0004-6361:20031659

193. Habart, E., Natta, A., Krügel, E.: PAHs in circumstellar disks around Herbig Ae/Be stars. A\&A, 427, 179-192 (2004). DOI 10.1051/0004-6361:20035916

194. Habart, E., Natta, A., Testi, L., Carbillet, M.: Spatially resolved PAH emission in the inner disks of Herbig Ae/Be stars. A\&A, 449, 1067-1075 (2006). DOI $10.1051 /$ 0004-6361:20052994

195. Hartogh, P., Lis, D.C., Bockelée-Morvan, D., de Val-Borro, M., Biver, N., Küppers, M., Emprechtinger, M., Bergin, E.A., Crovisier, J., Rengel, M., Moreno, R., Szutowicz, S., Blake, G.A.: Ocean-like water in the Jupiter-family comet 103P/Hartley 2. Nature, 478, 218-220 (2011). DOI 10.1038/nature10519

196. Hasegawa, T.I., Herbst, E., Leung, C.M.: Models of gas-grain chemistry in dense interstellar clouds with complex organic molecules. ApJS, 82, 167-195 (1992). DOI $10.1086 / 191713$

197. Hassel, G.E., Harada, N., Herbst, E.: Carbon-chain Species in Warm-up Models. ApJ, 743, 182 (2011). DOI 10.1088/0004-637X/743/2/182

198. Hassel, G.E., Herbst, E., Garrod, R.T.: Modeling the Lukewarm Corino Phase: Is L1527 unique? ApJ, 681, 1385-1395 (2008). DOI 10.1086/588185 
199. Heinzeller, D., Nomura, H., Walsh, C., Millar, T.J.: Chemical Evolution of Protoplanetary Disks - The Effects of Viscous Accretion, Turbulent Mixing, and Disk Winds. ApJ, 731, 115 (2011). DOI 10.1088/0004-637X/731/2/115

200. Hennebelle, P., Fromang, S.: Magnetic processes in a collapsing dense core. I. Accretion and ejection. A\&A, 477, 9-24 (2008). DOI 10.1051/0004-6361:20078309

201. Henning, T., Semenov, D., Guilloteau, S., Dutrey, A., Hersant, F., Wakelam, V., Chapillon, E., Launhardt, R., Piétu, V., Schreyer, K.: Chemistry in Disks. III. Photochemistry and X-ray Driven Chemistry Probed by the Ethynyl Radical $(\mathrm{CCH})$ in DM Tau, LkCa 15, and MWC 480. ApJ, 714, 1511-1520 (2010). DOI 10.1088/0004-637X/ $714 / 2 / 1511$

202. Herbst, E., Klemperer, W.: The Formation and Depletion of Molecules in Dense Interstellar Clouds. ApJ, 185, 505-534 (1973). DOI 10.1086/152436

203. Herbst, E., van Dishoeck, E.F.: Complex Organic Interstellar Molecules. ARA\&A, 47, 427-480 (2009). DOI 10.1146/annurev-astro-082708-101654

204. Hersant, F., Wakelam, V., Dutrey, A., Guilloteau, S., Herbst, E.: Cold CO in circumstellar disks. On the effects of photodesorption and vertical mixing. A\&A, 493, L49-L52 (2009). DOI 10.1051/0004-6361:200811082

205. Hily-Blant, P., Walmsley, M., Pineau Des Forêts, G., Flower, D.: Nitrogen chemistry and depletion in starless cores. A\&A, 513, A41 (2010). DOI 10.1051/0004-6361/ 200913200

206. Hirahara, Y., Suzuki, H., Yamamoto, S., Kawaguchi, K., Kaifu, N., Ohishi, M., Takano, S., Ishikawa, S.I., Masuda, A.: Mapping observations of sulfur-containing carbon-chain molecules in Taurus Molecular Cloud 1 (TMC-1). ApJ, 394, 539-551 (1992). DOI $10.1086 / 171605$

207. Hiraoka, K., Miyagoshi, T., Takayama, T., Yamamoto, K., Kihara, Y.: Gas-Grain Processes for the Formation of $\mathrm{CH} 4$ and $\mathrm{H} 2 \mathrm{O}$ : Reactions of $\mathrm{H}$ Atoms with $\mathrm{C}$, O, and $\mathrm{CO}$ in the Solid Phase at $12 \mathrm{~K}$. ApJ, 498, 710 (1998). DOI 10.1086/305572

208. Hiraoka, K., Sato, T., Sato, S., Sogoshi, N., Yokoyama, T., Takashima, H., Kitagawa, S.: Formation of Formaldehyde by the Tunneling Reaction of $\mathrm{H}$ with Solid CO at 10 K Revisited. ApJ, 577, 265-270 (2002). DOI 10.1086/342132

209. Hogerheijde, M.R., Bergin, E.A., Brinch, C., Cleeves, L.I., Fogel, J.K.J., Blake, G.A., Dominik, C., Lis, D.C., Melnick, G., Neufeld, D., Panić, O., Pearson, J.C., Kristensen, L., Yıldız, U.A., van Dishoeck, E.F.: Detection of the Water Reservoir in a Forming Planetary System. Science 334, 338- (2011). DOI 10.1126/science.1208931

210. Hollenbach, D., Kaufman, M.J., Bergin, E.A., Melnick, G.J.: Water, $\mathrm{O}_{2}$, and Ice in Molecular Clouds. ApJ, 690, 1497-1521 (2009). DOI 10.1088/0004-637X/690/2/1497

211. Hollenbach, D., McKee, C.F.: Molecule formation and infrared emission in fast interstellar shocks. III - Results for J shocks in molecular clouds. ApJ, 342, 306-336 (1989). DOI $10.1086 / 167595$

212. Hollenbach, D., Salpeter, E.E.: Surface Recombination of Hydrogen Molecules. ApJ, 163, 155 (1971). DOI 10.1086/150754

213. Honda, M., Inoue, A.K., Fukagawa, M., Oka, A., Nakamoto, T., Ishii, M., Terada, H., Takato, N., Kawakita, H., Okamoto, Y.K., Shibai, H., Tamura, M., Kudo, T., Itoh, Y.: Detection of Water Ice Grains on the Surface of the Circumstellar Disk Around HD 142527. ApJL, 690, L110-L113 (2009). DOI 10.1088/0004-637X/690/2/L110

214. Horner, J., Mousis, O., Hersant, F.: Constraints on the Formation Regions of Comets from their D:H Ratios. Earth Moon and Planets 100, 43-56 (2007). DOI 10.1007/ s11038-006-9096-4

215. Hughes, A.M., Andrews, S.M., Espaillat, C., Wilner, D.J., Calvet, N., D'Alessio, P., Qi, C., Williams, J.P., Hogerheijde, M.R.: A Spatially Resolved Inner Hole in the Disk Around GM Aurigae. ApJ, 698, 131-142 (2009). DOI 10.1088/0004-637X/698/1/131

216. Hughes, A.M., Wilner, D.J., Andrews, S.M., Williams, J.P., Su, K.Y.L., Murray-Clay, R.A., Qi, C.: Resolved Submillimeter Observations of the HR 8799 and HD 107146 Debris Disks. ApJ, 740, 38 (2011). DOI 10.1088/0004-637X/740/1/38

217. Ilee, J.D., Boley, A.C., Caselli, P., Durisen, R.H., Hartquist, T.W., Rawlings, J.M.C.: Chemistry in a gravitationally unstable protoplanetary disc. MNRAS, 417, 2950-2961 (2011). DOI 10.1111/j.1365-2966.2011.19455.x 
218. Ilgner, M., Henning, T., Markwick, A.J., Millar, T.J.: Transport processes and chemical evolution in steady accretion disk flows. A\&A, 415, 643-659 (2004). DOI 10.1051/ 0004-6361:20034061

219. Ioppolo, S., Cuppen, H.M., Romanzin, C., van Dishoeck, E.F., Linnartz, H.: Laboratory Evidence for Efficient Water Formation in Interstellar Ices. ApJ, 686, 1474-1479 (2008). DOI 10.1086/591506

220. Ioppolo, S., Palumbo, M.E., Baratta, G.A., Mennella, V.: Formation of interstellar solid $\mathrm{CO}\{-2\}$ after energetic processing of icy grain mantles. A\&A, 493, 1017-1028 (2009). DOI 10.1051/0004-6361:200809769

221. Ioppolo, S., van Boheemen, Y., Cuppen, H.M., van Dishoeck, E.F., Linnartz, H.: Surface formation of $\mathrm{CO}_{2}$ ice at low temperatures. MNRAS, 413, 2281-2287 (2011). DOI 10.1111/j.1365-2966.2011.18306.x

222. Irvine, W.M., Friberg, P., Kaifu, N., Kawaguchi, K., Kitamura, Y., Matthews, H.E., Minh, Y., Saito, S., Ukita, N., Yamamoto, S.: Observations of some oxygen-containing and sulfur-containing organic molecules in cold dark clouds. ApJ, 342, 871-875 (1989). DOI 10.1086/167643

223. Isella, A., Testi, L., Natta, A.: Large dust grains in the inner region of circumstellar disks. A\&A, 451, 951-959 (2006). DOI 10.1051/0004-6361:20054647

224. Jehin, E., Manfroid, J., Hutsemékers, D., Arpigny, C., Zucconi, J.M.: Isotopic Ratios in Comets: Status and Perspectives. Earth Moon and Planets 105, 167-180 (2009). DOI 10.1007/s11038-009-9322-y

225. Jing, D., He, J., Brucato, J., De Sio, A., Tozzetti, L., Vidali, G.: On Water Formation in the Interstellar Medium: Laboratory Study of the O+D Reaction on Surfaces. ApJL, 741, L9 (2011). DOI 10.1088/2041-8205/741/1/L9

226. Jones, A.P., Williams, D.A.: Time-dependent sticking coefficients and mantle growth on interstellar grains. MNRAS, 217, 413-421 (1985)

227. Joos, M., Hennebelle, P., Ciardi, A.: Protostellar disk formation and transport of angular momentum during magnetized core collapse. A\&A, 543, A128 (2012). DOI 10.1051/0004-6361/201118730

228. Jørgensen, J.K., Bourke, T.L., Myers, P.C., Di Francesco, J., van Dishoeck, E.F., Lee, C.F., Ohashi, N., Schöier, F.L., Takakuwa, S., Wilner, D.J., Zhang, Q.: PROSAC: A Submillimeter Array Survey of Low-Mass Protostars. I. Overview of Program: Envelopes, Disks, Outflows, and Hot Cores. ApJ, 659, 479-498 (2007). DOI $10.1086 / 512230$

229. Jørgensen, J.K., Bourke, T.L., Nguyen Luong, Q., Takakuwa, S.: Arcsecond resolution images of the chemical structure of the low-mass protostar IRAS 16293-2422. An overview of a large molecular line survey from the Submillimeter Array. A\&A, 534, A100 (2011). DOI 10.1051/0004-6361/201117139

230. Jørgensen, J.K., Favre, C., Bisschop, S.E., Bourke, T.L., van Dishoeck, E.F., Schmalzl, M.: Detection of the Simplest Sugar, Glycolaldehyde, in a Solar-type Protostar with ALMA. ApJL, 757, L4 (2012). DOI 10.1088/2041-8205/757/1/L4

231. Jørgensen, J.K., Schöier, F.L., van Dishoeck, E.F.: Physical structure and CO abundance of low-mass protostellar envelopes. A\&A, 389, 908-930 (2002). DOI 10.1051/ 0004-6361:20020681

232. Jørgensen, J.K., Schöier, F.L., van Dishoeck, E.F.: Molecular freeze-out as a tracer of the thermal and dynamical evolution of pre- and protostellar cores. A\&A, 435, 177-182 (2005). DOI 10.1051/0004-6361:20042092

233. Jørgensen, J.K., van Dishoeck, E.F.: The $\mathrm{HDO} / \mathrm{H}_{2} \mathrm{O}$ Ratio in Gas in the Inner Regions of a Low-mass Protostar. ApJL, 725, L172-L175 (2010). DOI 10.1088/2041-8205/725/ 2/L172

234. Jørgensen, J.K., van Dishoeck, E.F.: Water Vapor in the Inner 25 AU of a Young Disk Around a Low-Mass Protostar. ApJL, 710, L72-L76 (2010). DOI 10.1088/2041-8205/ $710 / 1 / \mathrm{L} 72$

235. Jørgensen, J.K., van Dishoeck, E.F., Visser, R., Bourke, T.L., Wilner, D.J., Lommen D., Hogerheijde, M.R., Myers, P.C.: PROSAC: a submillimeter array survey of lowmass protostars. II. The mass evolution of envelopes, disks, and stars from the Class 0 through I stages. A\&A, 507, 861-879 (2009). DOI 10.1051/0004-6361/200912325 
236. Kaifu, N., Ohishi, M., Kawaguchi, K., Saito, S., Yamamoto, S., Miyaji, T., Miyazawa, K., Ishikawa, S.I., Noumaru, C., Harasawa, S., Okuda, M., Suzuki, H.: A 8.8-50GHz Complete Spectral Line Survey toward TMC-1 I. Survey Data. PASJ, 56, 69-173 (2004)

237. Kamp, I.: Evolution of PAHs in Protoplanetary Disks. In: C. Joblin, A.G.G.M. Tielens (eds.) EAS Publications Series, EAS Publications Series, vol. 46, pp. 271-283 (2011). DOI $10.1051 /$ eas $/ 1146029$

238. Kamp, I., Woitke, P., Pinte, C., Tilling, I., Thi, W.F., Menard, F., Duchene, G., Augereau, J.C.: Continuum and line modelling of discs around young stars. II Line diagnostics for GASPS from the DENT grid. A\&A, 532, A85 (2011). DOI 10.1051/0004-6361/201016399

239. Kaufman, M.J., Neufeld, D.A.: Far-Infrared Water Emission from Magnetohydrodynamic Shock Waves. ApJ, 456, 611 (1996). DOI 10.1086/176683

240. Kavelaars, J.J., Mousis, O., Petit, J.M., Weaver, H.A.: On the Formation Location of Uranus and Neptune as Constrained by Dynamical and Chemical Models of Comets. ApJL, 734, L30 (2011). DOI 10.1088/2041-8205/734/2/L30

241. Kawamoto, T.: Experimental constraints on differentiation and $\mathrm{H}_{2} \mathrm{O}$ abundance of calc-alkaline magmas. Earth and Planetary Science Letters 144, 577-589 (1996). DOI 10.1016/S0012-821X(96)00182-3

242. Keller, L.D., Sloan, G.C., Forrest, W.J., Ayala, S., D'Alessio, P., Shah, S., Calvet, N., Najita, J., Li, A., Hartmann, L., Sargent, B., Watson, D.M., Chen, C.H.: PAH Emission from Herbig Ae/Be Stars. ApJ, 684, 411-429 (2008). DOI 10.1086/589818

243. Keller, L.P., Messenger, S., Flynn, G.J., Clemett, S., Wirick, S., Jacobsen, C.: The nature of molecular cloud material in interplanetary dust. Geochimica et Cosmochimica Acta68, 2577-2589 (2004). DOI 10.1016/j.gca.2003.10.044

244. Kessler-Silacci, J.E., Hillenbrand, L.A., Blake, G.A., Meyer, M.R.: 8-13 $\mu \mathrm{m} \mathrm{Spec-}$ troscopy of Young Stellar Objects: Evolution of the Silicate Feature. ApJ, 622, 404-429 (2005). DOI $10.1086 / 427793$

245. Keto, E., Caselli, P.: The Different Structures of the Two Classes of Starless Cores. ApJ, 683, 238-247 (2008). DOI 10.1086/589147

246. Keto, E., Caselli, P.: Dynamics and depletion in thermally supercritical starless cores MNRAS, 402, 1625-1634 (2010). DOI 10.1111/j.1365-2966.2009.16033.x

247. Kita, N.T., Nagahara, H., Togashi, S., Morishita, Y.: A short duration of chondrule formation in the solar nebula: evidence from ${ }^{\wedge} 2^{\wedge} 6 \mathrm{Al}$ in Semarkona ferromagnesian chondrules. Geochimica et Cosmochimica Acta64, 3913-3922 (2000). DOI 10.1016/ S0016-7037(00)00488-9

248. Kita, N.T., Togashi, S., Morishita, Y., Terashima, S., Yurimoto, H.: Search for ${ }^{60} \mathrm{Ni}$ excesses in MET-78008 ureilite: An ion microprobe study. Antarctic Meteorite Research 11, 103 (1998)

249. Krasnopolsky, R., Li, Z.Y., Shang, H.: Disk Formation in Magnetized Clouds Enabled by the Hall Effect. ApJ, 733, 54 (2011). DOI 10.1088/0004-637X/733/1/54

250. Kristensen, L.E., van Dishoeck, E.F., Bergin, E.A., Visser, R., Yıldı, U.A., San JoseGarcia, I., Jørgensen, J.K., Herczeg, G.J., Johnstone, D., Wampfler, S.F., Benz, A.O., Bruderer, S., Cabrit, S., Caselli, P., Doty, S.D., Harsono, D., Herpin, F., Hogerheijde, M.R., Karska, A., van Kempen, T.A., Liseau, R., Nisini, B., Tafalla, M., van der Tak, F., Wyrowski, F.: Water in star-forming regions with Herschel (WISH). II. Evolution of $557 \mathrm{GHz} 1_{10}-1_{01}$ emission in low-mass protostars. A\&A, 542, A8 (2012). DOI $10.1051 / 0004-6361 / 201118146$

251. Kristensen, L.E., van Dishoeck, E.F., Tafalla, M., Bachiller, R., Nisini, B., Liseau, R., Yıldız, U.A.: Water in low-mass star-forming regions with Herschel (WISH-LM). High-velocity $\mathrm{H}_{2} \mathrm{O}$ bullets in L1448-MM observed with HIFI. A\&A, 531, L1 (2011). DOI 10.1051/0004-6361/201116975

252. Kristensen, L.E., Visser, R., van Dishoeck, E.F., Yıldız, U.A., Doty, S.D., Herczeg, G.J., Liu, F.C., Parise, B., Jørgensen, J.K., van Kempen, T.A., Brinch, C., Wampfler, S.F., Bruderer, S., Benz, A.O., Hogerheijde, M.R., Deul, E., Bachiller, R., Baudry, A., Benedettini, M., Bergin, E.A., Bjerkeli, P., Blake, G.A., Bontemps, S., Braine, J., Caselli, P., Cernicharo, J., Codella, C., Daniel, F., de Graauw, T., di Giorgio, A.M., Dominik, C., Encrenaz, P., Fich, M., Fuente, A., Giannini, T., Goicoechea, J.R., 
Helmich, F., Herpin, F., Jacq, T., Johnstone, D., Kaufman, M.J., Larsson, B., Lis, D., Liseau, R., Marseille, M., McCoey, C., Melnick, G., Neufeld, D., Nisini, B., Olberg, M., Pearson, J.C., Plume, R., Risacher, C., Santiago-García, J., Saraceno, P., Shipman, R., Tafalla, M., Tielens, A.G.G.M., van der Tak, F., Wyrowski, F., Beintema, D., de Jonge, A., Dieleman, P., Ossenkopf, V., Roelfsema, P., Stutzki, J., Whyborn, N.: Water in lowmass star-forming regions with Herschel . HIFI spectroscopy of NGC 1333. A\&A, 521, L30 (2010). DOI 10.1051/0004-6361/201015100

253. Kwon, W., Looney, L.W., Mundy, L.G., Chiang, H.F., Kemball, A.J.: Grain Growth and Density Distribution of the Youngest Protostellar Systems. ApJ, 696, 841-852 (2009). DOI 10.1088/0004-637X/696/1/841

254. Laas, J.C., Garrod, R.T., Herbst, E., Widicus Weaver, S.L.: Contributions from Grain Surface and Gas Phase Chemistry to the Formation of Methyl Formate and Its Structural Isomers. ApJ, 728, 71 (2011). DOI 10.1088/0004-637X/728/1/71

255. Lada, C.J., Bergin, E.A., Alves, J.F., Huard, T.L.: The Dynamical State of Barnard 68: A Thermally Supported, Pulsating Dark Cloud. ApJ, 586, 286-295 (2003). DOI $10.1086 / 367610$

256. Lahuis, F., van Dishoeck, E.F., Boogert, A.C.A., Pontoppidan, K.M., Blake, G.A. Dullemond, C.P., Evans II, N.J., Hogerheijde, M.R., Jørgensen, J.K., Kessler-Silacci, J.E., Knez, C.: Hot Organic Molecules toward a Young Low-Mass Star: A Look at Inner Disk Chemistry. ApJL, 636, L145-L148 (2006). DOI 10.1086/500084

257. Lattelais, M., Pauzat, F., Ellinger, Y., Ceccarelli, C.: Interstellar Complex Organic Molecules and the Minimum Energy Principle. ApJL, 696, L133-L136 (2009). DOI 10.1088/0004-637X/696/2/L133

258. Laughlin, G., Bodenheimer, P.: Nonaxisymmetric evolution in protostellar disks. ApJ, 436, 335-354 (1994). DOI 10.1086/174909

259. Le Guillou, C., Rouzaud, J.N., Bonal, L., Quirico, E., Derenne, S., Remusat, L.: High resolution TEM of chondritic carbonaceous matter: Metamorphic evolution and heterogeneity. Meteoritics and Planetary Science 47, 345-362 (2012). DOI 10.1111/j.1945-5100.2012.01336.x

260. Lécuyer, C., Simon, L., Guy, F.: Comparison of carbon, nitrogen and water budgets on Venus and the Earth. Earth and Planetary Science Letters 181, 33-40 (2000). DOI 10.1016/S0012-821X(00)00195-3

261. Lee, J.E., Evans II, N.J., Bergin, E.A.: Comparisons of an Evolutionary Chemical Model with Other Models. ApJ, 631, 351-360 (2005). DOI 10.1086/432531

262. Lee, N., Williams, J.P., Cieza, L.A.: Protoplanetary Disk Masses in IC348: A Rapid Decline in the Population of Small Dust Grains After 1 Myr. ApJ, 736, 135 (2011). DOI 10.1088/0004-637X/736/2/135

263. Lee, T., Shu, F.H., Shang, H., Glassgold, A.E., Rehm, K.E.: Protostellar Cosmic Rays and Extinct Radioactivities in Meteorites. ApJ, 506, 898-912 (1998). DOI 10.1086/ 306284

264. Lefloch, B., Cabrit, S., Codella, C., Melnick, G., Cernicharo, J., Caux, E., Benedettini, M., Boogert, A., Caselli, P., Ceccarelli, C., Gueth, F., Hily-Blant, P., Lorenzani, A. Neufeld, D., Nisini, B., Pacheco, S., Pagani, L., Pardo, J.R., Parise, B., Salez, M., Schuster, K., Viti, S., Bacmann, A., Baudry, A., Bell, T., Bergin, E.A., Blake, G., Bottinelli, S., Castets, A., Comito, C., Coutens, A., Crimier, N., Dominik, C., Demyk, K., Encrenaz, P., Falgarone, E., Fuente, A., Gerin, M., Goldsmith, P., Helmich, F., Hennebelle, P., Henning, T., Herbst, E., Jacq, T., Kahane, C., Kama, M., Klotz, A., Langer, W., Lis, D., Lord, S., Maret, S., Pearson, J., Phillips, T., Saraceno, P., Schilke, P., Tielens, X., van der Tak, F., van der Wiel, M., Vastel, C., Wakelam, V., Walters, A., Wyrowski, F., Yorke, H., Bachiller, R., Borys, C., de Lange, G., Delorme, Y., Kramer, C., Larsson, B., Lai, R., Maiwald, F.W., Martin-Pintado, J., Mehdi, I., Ossenkopf, V., Siegel, P., Stutzki, J., Wunsch, J.H.: The CHESS spectral survey of star forming regions: Peering into the protostellar shock L1157-B1. II. Shock dynamics. A\&A, 518 , L113 (2010). DOI 10.1051/0004-6361/201014630

265. Lefloch, B., Castets, A., Cernicharo, J., Langer, W.D., Zylka, R.: Cores and cavities in NGC 1333. A\&A, 334, 269-279 (1998)

266. Lefloch, B., Cernicharo, J., Pacheco, S., Ceccarelli, C.: Shocked water in the Cepheus E protostellar outflow. A\&A, 527, L3 (2011). DOI 10.1051/0004-6361/201016247 
267. Leger, A., Jura, M., Omont, A.: Desorption from interstellar grains. A\&A, 144, 147$160(1985)$

268. Levison, H.F., Duncan, M.J., Brasser, R., Kaufmann, D.E.: Capture of the Sun's Oort Cloud from Stars in Its Birth Cluster. Science 329, 187-(2010). DOI 10.1126/science. 1187535

269. Licandro, J., Hargrove, K., Kelley, M., Campins, H., Ziffer, J., Alí-Lagoa, V., Fernández, Y., Rivkin, A.: 5-14 $\mu \mathrm{m}$ Spitzer spectra of Themis family asteroids. A\&A, 537, A73 (2012). DOI 10.1051/0004-6361/201118142

270. Lis, D.C., Wootten, A., Gerin, M., Roueff, E.: Nitrogen Isotopic Fractionation in Interstellar Ammonia. ApJL, 710, L49-L52 (2010). DOI 10.1088/2041-8205/710/1/L49

271. Liseau, R., Ceccarelli, C., Larsson, B., Nisini, B., White, G.J., Ade, P., Armand, C., Burgdorf, M., Caux, E., Cerulli, R., Church, S., Clegg, P.E., Digorgio, A., Furniss, I., Giannini, T., Glencross, W., Gry, C., King, K., Lim, T., Lorenzetti, D., Molinari, S., Naylor, D., Orfei, R., Saraceno, P., Sidher, S., Smith, H., Spinoglio, L., Swinyard, B., Texier, D., Tommasi, E., Trams, N., Unger, S.: Thermal H_2_O emission from the Herbig-Haro flow HH 54. A\&A, 315, L181-L184 (1996)

272. Liu, F.C., Parise, B., Kristensen, L., Visser, R., van Dishoeck, E.F., Güsten, R.: Water deuterium fractionation in the low-mass protostar NGC1333-IRAS2A. A\&A, 527, A19 (2011). DOI 10.1051/0004-6361/201015519

273. Loinard, L., Castets, A., Ceccarelli, C., Caux, E., Tielens, A.G.G.M.: Doubly Deuterated Molecular Species in Protostellar Environments. ApJL, 552, L163-L166 (2001). DOI 10.1086/320331

274. Lommen, D.J.P., van Dishoeck, E.F., Wright, C.M., Maddison, S.T., Min, M., Wilner, D.J., Salter, D.M., van Langevelde, H.J., Bourke, T.L., van der Burg, R.F.J., Blake, G.A.: Grain growth across protoplanetary discs: $10 \mu \mathrm{m}$ silicate feature versus millimetre slope. A\&A, 515, A77 (2010). DOI 10.1051/0004-6361/200913150

275. Looney, L.W., Mundy, L.G., Welch, W.J.: Unveiling the Circumstellar Envelope and Disk: A Subarcsecond Survey of Circumstellar Structures. ApJ, 529, 477-498 (2000). DOI $10.1086 / 308239$

276. Machida, M.N., Inutsuka, S.I., Matsumoto, T.: Effect of Magnetic Braking on Circumstellar Disk Formation in a Strongly Magnetized Cloud. PASJ, 63, 555- (2011)

277. Malbet, F., Benisty, M., de Wit, W.J., Kraus, S., Meilland, A., Millour, F., Tatulli, E., Berger, J.P., Chesneau, O., Hofmann, K.H., Isella, A., Natta, A., Petrov, R.G., Preibisch, T., Stee, P., Testi, L., Weigelt, G., Antonelli, P., Beckmann, U., Bresson, Y., Chelli, A., Dugué, M., Duvert, G., Gennari, S., Glück, L., Kern, P., Lagarde, S., Le Coarer, E., Lisi, F., Perraut, K., Puget, P., Rantakyrö, F., Robbe-Dubois, S., Roussel, A., Zins, G., Accardo, M., Acke, B., Agabi, K., Altariba, E., Arezki, B., Aristidi, E., Baffa, C., Behrend, J., Blöcker, T., Bonhomme, S., Busoni, S., Cassaing, F., Clausse, J.M., Colin, J., Connot, C., Delboulbé, A., Domiciano de Souza, A., Driebe, T., Feautrier, P., Ferruzzi, D., Forveille, T., Fossat, E., Foy, R., Fraix-Burnet, D., Gallardo, A., Giani, E., Gil, C., Glentzlin, A., Heiden, M., Heininger, M., Hernandez Utrera, O., Kamm, D., Kiekebusch, M., Le Contel, D., Le Contel, J.M., Lesourd, T., Lopez, B., Lopez, M., Magnard, Y., Marconi, A., Mars, G., Martinot-Lagarde, G., Mathias, P., Mège, P., Monin, J.L., Mouillet, D., Mourard, D., Nussbaum, E., Ohnaka, K., Pacheco, J., Perrier, C., Rabbia, Y., Rebattu, S., Reynaud, F., Richichi, A., Robini, A., Sacchettini, M., Schertl, D., Schöller, M., Solscheid, W., Spang, A., Stefanini, P., Tallon, M., Tallon-Bosc, I., Tasso, D., Vakili, F., von der Lühe, O., Valtier, J.C., Vannier, M., Ventura, N.: Disk and wind interaction in the young stellar object ¡ASTROBJ ¿MWC 297i/ASTROBJ $i$ spatially resolved with AMBER/VLTI. A\&A, 464, 43-53 (2007). DOI 10.1051/0004-6361:20053924

278. Mandell, A.M., Bast, J., van Dishoeck, E.F., Blake, G.A., Salyk, C., Mumma, M.J., Villanueva, G.: First Detection of Near-infrared Line Emission from Organics in Young Circumstellar Disks. ApJ, 747, 92 (2012). DOI 10.1088/0004-637X/747/2/92

279. Manfroid, J., Jehin, E., Hutsemékers, D., Cochran, A., Zucconi, J.M., Arpigny, C., Schulz, R., Stüwe, J.A., Ilyin, I.: The CN isotopic ratios in comets. A\&A, 503, 613624 (2009). DOI 10.1051/0004-6361/200911859

280. Marcelino, N., Brünken, S., Cernicharo, J., Quan, D., Roueff, E., Herbst, E., Thaddeus, P.: The puzzling behavior of HNCO isomers in molecular clouds. A\&A, 516, A105 (2010). DOI 10.1051/0004-6361/200913806 
281. Marcelino, N., Cernicharo, J., Agúndez, M., Roueff, E., Gerin, M., Martín-Pintado, J., Mauersberger, R., Thum, C.: Discovery of Interstellar Propylene $\left(\mathrm{CH}_{2} \mathrm{CHCH}_{3}\right)$ : Missing Links in Interstellar Gas-Phase Chemistry. ApJL, 665, L127-L130 (2007). DOI 10.1086/521398

282. Marcelino, N., Cernicharo, J., Roueff, E., Gerin, M., Mauersberger, R.: Deuterated Thioformaldehyde in the Barnard 1 Cloud. ApJ, 620, 308-320 (2005). DOI 10.1086/ 426934

283. Marhas, K.K., Goswami, J.N., Davis, A.M.: Short-Lived Nuclides in Hibonite Grains from Murchison: Evidence for Solar System Evolution. Science 298, 2182-2185 (2002). DOI 10.1126/science.1078322

284. Marshall, C.P., Emry, J.R., Olcott Marshall, A.: Haematite pseudomicrofossils present in the 3.5-billion-year-old Apex Chert. Nature Geoscience 4, 240-243 (2011). DOI $10.1038 /$ ngeo1084

285. Marty, B.: The origins and concentrations of water, carbon, nitrogen and noble gases on Earth. Earth and Planetary Science Letters 313, 56-66 (2012). DOI 10.1016/j. epsl.2011.10.040

286. Marty, B., Zimmermann, L., Burnard, P.G., Wieler, R., Heber, V.S., Burnett, D.L., Wiens, R.C., Bochsler, P.: Nitrogen isotopes in the recent solar wind from the analysis of Genesis targets: Evidence for large scale isotope heterogeneity in the early solar system. Geochimica et Cosmochimica Acta74, 340-355 (2010)

287. Mathews, G.S., Dent, W.R.F., Williams, J.P., Howard, C.D., Meeus, G., Riaz, B., Roberge, A., Sandell, G., Vandenbussche, B., Duchêne, G., Kamp, I., Ménard, F., Montesinos, B., Pinte, C., Thi, W.F., Woitke, P., Alacid, J.M., Andrews, S.M., Ardila, D.R., Aresu, G., Augereau, J.C., Barrado, D., Brittain, S., Ciardi, D.R., Danchi, W., Eiroa, C., Fedele, D., Grady, C.A., de Gregorio-Monsalvo, I., Heras, A., Huelamo, N., Krivov, A., Lebreton, J., Liseau, R., Martin-Zaidi, C., Mendigutía, I., Mora, A., Morales-Calderon, M., Nomura, H., Pantin, E., Pascucci, I., Phillips, N., Podio, L., Poelman, D.R., Ramsay, S., Rice, K., Riviere-Marichalar, P., Solano, E., Tilling, I., Walker, H., White, G.J., Wright, G.: GAS in Protoplanetary Systems (GASPS). I. First results. A\&A, 518, L127 (2010). DOI 10.1051/0004-6361/201014595

288. Matrajt, G., Messenger, S., Brownlee, D., Joswiak, D.: Diverse forms of primordial organic matter identified in interplanetary dust particles. Meteoritics and Planetary Science 47, 525-549 (2012). DOI 10.1111/j.1945-5100.2011.01310.x

289. Matthews, H.E., Friberg, P., Irvine, W.M.: The detection of acetaldehyde in cold dust clouds. ApJ, 290, 609-614 (1985). DOI 10.1086/163018

290. Matthews, H.E., Sears, T.J.: The detection of vinyl cyanide in TMC-1. ApJ, 272, 149-153 (1983). DOI 10.1086/161271

291. McCarthy, M.C., Gottlieb, C.A., Gupta, H., Thaddeus, P.: Laboratory and Astronomical Identification of the Negative Molecular Ion $\mathrm{C}_{6} \mathrm{H}^{-}$. ApJL, 652, L141-L144 (2006). DOI 10.1086/510238

292. McKeegan, K.D., Chaussidon, M., Robert, F.: Incorporation of Short-Lived ${ }^{10}$ Be in a Calcium-Aluminum-Rich Inclusion from the Allende Meteorite. Science 289, 13341337 (2000). DOI 10.1126/science.289.5483.1334

293. Meibom, A., Krot, A.N., Robert, F., Mostefaoui, S., Russell, S.S., Petaev, M.I. Gounelle, M.: Nitrogen and Carbon Isotopic Composition of the Sun Inferred from a High-Temperature Solar Nebular Condensate. ApJL, 656, L33-L36 (2007). DOI $10.1086 / 512052$

294. Meier, R., Owen, T.C., Jewitt, D.C., Matthews, H.E., Senay, M., Biver, N., BockeleeMorvan, D., Crovisier, J., Gautier, D.: Deuterium in Comet C/1995 O1 (Hale-Bopp): Detection of DCN. Science 279, 1707 (1998). DOI 10.1126/science.279.5357.1707

295. Meijerink, R., Glassgold, A.E., Najita, J.R.: Atomic Diagnostics of X-Ray-Irradiated Protoplanetary Disks. ApJ, 676, 518-531 (2008). DOI 10.1086/527411

296. Mellon, R.R., Li, Z.Y.: Magnetic Braking and Protostellar Disk Formation: The Ideal MHD Limit. ApJ, 681, 1356-1376 (2008). DOI 10.1086/587542

297. Merín, B., Augereau, J.C., van Dishoeck, E.F., Kessler-Silacci, J., Dullemond, C.P., Blake, G.A., Lahuis, F., Brown, J.M., Geers, V.C., Pontoppidan, K.M., Comerón, F., Frasca, A., Guieu, S., Alcalá, J.M., Boogert, A.C.A., Evans II, N.J., D'Alessio, P., Mundy, L.G., Chapman, N.: Abundant Crystalline Silicates in the Disk of a Very Low Mass Star. ApJ, 661, 361-367 (2007). DOI 10.1086/513092 
298. Messenger, S.: Identification of molecular-cloud material in interplanetary dust particles. Nature, 404, 968-971 (2000)

299. Milam, S.N., Charnley, S.B.: Observations of Nitrogen Fractionation in Prestellar Cores: Nitriles Tracing Interstellar Chemistry. In: Lunar and Planetary Institute Science Conference Abstracts, Lunar and Planetary Inst. Technical Report, vol. 43, p. $2618(2012)$

300. Miyauchi, N., Hidaka, H., Chigai, T., Nagaoka, A., Watanabe, N., Kouchi, A.: Formation of hydrogen peroxide and water from the reaction of cold hydrogen atoms with solid oxygen at $10 \mathrm{~K}$. Chemical Physics Letters 456, 27-30 (2008). DOI 10.1016/j.cplett.2008.02.095

301. Modica, P., Palumbo, M.E.: Formation of methyl formate after cosmic ion irradiation of icy grain mantles. A\&A, 519, A22 (2010). DOI 10.1051/0004-6361/201014101

302. Mokrane, H., Chaabouni, H., Accolla, M., Congiu, E., Dulieu, F., Chehrouri, M., Lemaire, J.L.: Experimental Evidence for Water Formation Via Ozone Hydrogenation on Dust Grains at 10 K. ApJL, 705, L195-L198 (2009). DOI 10.1088/0004-637X/705/ $2 /$ L195

303. Morbidelli, A., Chambers, J., Lunine, J.I., Petit, J.M., Robert, F., Valsecchi, G.B., Cyr, K.E.: Source regions and time scales for the delivery of water to Earth. Meteoritics and Planetary Science 35, 1309-1320 (2000). DOI 10.1111/j.1945-5100.2000.tb01518.x

304. Mouschovias, T.C.: Ambipolar diffusion in interstellar clouds - A new solution. ApJ, 228, 475-481 (1979). DOI 10.1086/156868

305. Moynier, F., Blichert-Toft, J., Wang, K., Herzog, G.F., Albarede, F.: The Elusive ${ }^{60} \mathrm{Fe}$ in the Solar Nebula. ApJ, 741, 71 (2011). DOI 10.1088/0004-637X/741/2/71

306. Muñoz Caro, G.M., Meierhenrich, U., Schutte, W.A., Thiemann, W.H.P., Greenberg, J.M.: UV-photoprocessing of interstellar ice analogs: Detection of hexamethylenetetramine-based species. A\&A, 413, 209-216 (2004). DOI 10.1051/ 0004-6361:20031447

307. Muñoz Caro, G.M., Meierhenrich, U.J., Schutte, W.A., Barbier, B., Arcones Segovia, A., Rosenbauer, H., Thiemann, W.H.P., Brack, A., Greenberg, J.M.: Amino acids from ultraviolet irradiation of interstellar ice analogues. Nature, 416, 403-406 (2002)

308. Müller, H.S.P., Schlöder, F., Stutzki, J., Winnewisser, G.: The Cologne Database for Molecular Spectroscopy, CDMS: a useful tool for astronomers and spectroscopists. Journal of Molecular Structure, 742, 215-227 (2005). DOI 10.1016/j.molstruc.2005. 01.027

309. Mumma, M.J., Charnley, S.B.: The Chemical Composition of Comets: Emerging Taxonomies and Natal Heritage. ARA\&A, 49, 471-524 (2011). DOI 10.1146/ annurev-astro-081309-130811

310. Murakawa, K., Tamura, M., Nagata, T.: 1-4 Micron Spectrophotometry of Dust in the Taurus Dark Cloud: Water Ice Distribution in Heiles Cloud 2. ApJS, 128, 603-613 (2000). DOI 10.1086/313387

311. Muzerolle, J., Calvet, N., Hartmann, L., D'Alessio, P.: Unveiling the Inner Disk Structure of T Tauri Stars. ApJL, 597, L149-L152 (2003). DOI 10.1086/379921

312. Nakamura, T., Noguchi, T., Tanaka, M., Zolensky, M.E., Kimura, M., Tsuchiyama, A., Nakato, A., Ogami, T., Ishida, H., Uesugi, M., Yada, T., Shirai, K., Fujimura, A., Okazaki, R., Sandford, S.A., Ishibashi, Y., Abe, M., Okada, T., Ueno, M., Mukai, T., Yoshikawa, M., Kawaguchi, J.: Itokawa Dust Particles: A Direct Link Between S-Type Asteroids and Ordinary Chondrites. Science 333, 1113- (2011). DOI 10.1126/science. 1207758

313. Natta, A., Prusti, T., Neri, R., Wooden, D., Grinin, V.P., Mannings, V.: A reconsideration of disk properties in Herbig Ae stars. A\&A, 371, 186-197 (2001). DOI 10.1051/0004-6361:20010334

314. Natta, A., Testi, L., Calvet, N., Henning, T., Waters, R., Wilner, D.: Dust in Protoplanetary Disks: Properties and Evolution. Protostars and Planets V pp. 767-781 (2007)

315. Nguyen, A.N., Stadermann, F.J., Zinner, E., Stroud, R.M., Alexander, C.M.O., Nittler, L.R.: Characterization of Presolar Silicate and Oxide Grains in Primitive Carbonaceous Chondrites. ApJ, 656, 1223-1240 (2007). DOI 10.1086/510612 
316. Nisini, B., Benedettini, M., Giannini, T., Codella, C., Lorenzetti, D., di Giorgio, A.M., Richer, J.S.: Far infrared mapping of the gas cooling along the L1448 outflow. A\&A, 360, 297-310 (2000)

317. Noble, J.A., Dulieu, F., Congiu, E., Fraser, H.J.: $\mathrm{CO}_{2}$ Formation in Quiescent Clouds: An Experimental Study of the CO + OH Pathway. ApJ, 735, 121 (2011). DOI 10.1088/0004-637X/735/2/121

318. Oba, Y., Watanabe, N., Hama, T., Kuwahata, K., Hidaka, H., Kouchi, A.: Water Formation through a Quantum Tunneling Surface Reaction, $\mathrm{OH}+\mathrm{H}_{2}$, at $10 \mathrm{~K}$. ApJ, 749, 67 (2012). DOI 10.1088/0004-637X/749/1/67

319. Oba, Y., Watanabe, N., Kouchi, A., Hama, T., Pirronello, V.: Experimental Study of $\mathrm{CO}_{2}$ Formation by Surface Reactions of Non-energetic OH Radicals with CO Molecules. ApJL, 712, L174-L178 (2010). DOI 10.1088/2041-8205/712/2/L174

320. Öberg, K.I., Garrod, R.T., van Dishoeck, E.F., Linnartz, H.: Formation rates of complex organics in UV irradiated CH_3OH-rich ices. I. Experiments. A\&A, 504, 891-913 (2009). DOI 10.1051/0004-6361/200912559

321. Öberg, K.I., Linnartz, H., Visser, R., van Dishoeck, E.F.: Photodesorption of Ices. II. $\mathrm{H}_{2} \mathrm{O}$ and $\mathrm{D}_{2} \mathrm{O}$. ApJ, 693, 1209-1218 (2009). DOI 10.1088/0004-637X/693/2/1209

322. Öberg, K.I., Qi, C., Fogel, J.K.J., Bergin, E.A., Andrews, S.M., Espaillat, C., van Kempen, T.A., Wilner, D.J., Pascucci, I.: The Disk Imaging Survey of Chemistry with SMA. I. Taurus Protoplanetary Disk Data. ApJ, 720, 480-493 (2010). DOI 10.1088/0004-637X/720/1/480

323. Öberg, K.I., Qi, C., Fogel, J.K.J., Bergin, E.A., Andrews, S.M., Espaillat, C., Wilner, D.J., Pascucci, I., Kastner, J.H.: Disk Imaging Survey of Chemistry with SMA. II. Southern Sky Protoplanetary Disk Data and Full Sample Statistics. ApJ, 734, 98 (2011). DOI 10.1088/0004-637X/734/2/98

324. Öberg, K.I., Qi, C., Wilner, D.J., Andrews, S.M.: The Ionization Fraction in the DM Tau Protoplanetary Disk. ApJ, 743, 152 (2011). DOI 10.1088/0004-637X/743/2/152

325. Öberg, K.I., van der Marel, N., Kristensen, L.E., van Dishoeck, E.F.: Complex Molecules toward Low-mass Protostars: The Serpens Core. ApJ, 740, 14 (2011). DOI 10.1088/0004-637X/740/1/14

326. Öberg, K.I., van Dishoeck, E.F., Linnartz, H.: Photodesorption of ices I: CO, $\mathrm{N}_{2}$, and $\mathrm{CO}_{2}$. A\&A, 496, 281-293 (2009). DOI 10.1051/0004-6361/200810207

327. Öberg, K.I., van Dishoeck, E.F., Linnartz, H., Andersson, S.: The Effect of $\mathrm{H}_{2} \mathrm{O}$ on Ice Photochemistry. ApJ, 718, 832-840 (2010). DOI 10.1088/0004-637X/718/2/832

328. Ohishi, M., Kaifu, N.: Chemical and physical evolution of dark clouds. Molecular spectral line survey toward TMC-1. Faraday Discussions 109, 205 (1998). DOI $10.1039 / \mathrm{a} 801058 \mathrm{~g}$

329. Oliveira, C.M., Hébrard, G., Howk, J.C., Kruk, J.W., Chayer, P., Moos, H.W.: Interstellar Deuterium, Nitrogen, and Oxygen Abundances toward GD 246, WD 2331-475, HZ 21, and Lanning 23: Results from the FUSE Mission. ApJ, 587, 235-255 (2003). DOI 10.1086/368019

330. Olofsson, J., Juhász, A., Henning, T., Mutschke, H., Tamanai, A., Moór, A., Ábrahám, P.: Transient dust in warm debris disks. Detection of Fe-rich olivine grains. A\&A, 542, A90 (2012). DOI 10.1051/0004-6361/201118735

331. Ossenkopf, V., Henning, T.: Dust opacities for protostellar cores. A\&A, 291, 943-959 (1994)

332. Owen, T., Bar-Nun, A.: Comets, impacts and atmospheres. Icarus, 116, 215-226 (1995). DOI 10.1006/icar.1995.1122

333. Owen, T., Mahaffy, P.R., Niemann, H.B., Atreya, S., Wong, M.: Protosolar Nitrogen. ApJL, 553, L77-L79 (2001). DOI 10.1086/320501

334. Padovani, M., Galli, D.: Effects of magnetic fields on the cosmic-ray ionization of molecular cloud cores. A\&A, 530, A109 (2011). DOI 10.1051/0004-6361/201116853

335. Pagani, L., Bacmann, A., Cabrit, S., Vastel, C.: Depletion and low gas temperature in the L183 (=L134N) prestellar core: the $\mathrm{N}_{2} \mathrm{H}^{\wedge}+-\mathrm{N}_{2} \mathrm{D}^{+}$tool. A\&A, 467, 179-186 (2007). DOI 10.1051/0004-6361:20066670

336. Pagani, L., Steinacker, J., Bacmann, A., Stutz, A., Henning, T.: The Ubiquity of Micrometer-Sized Dust Grains in the Dense Interstellar Medium. Science 329, 1622(2010). DOI 10.1126/science.1193211 
337. Pagani, L., Vastel, C., Hugo, E., Kokoouline, V., Greene, C.H., Bacmann, A. Bayet, E., Ceccarelli, C., Peng, R., Schlemmer, S.: Chemical modeling of $¡$ AS-

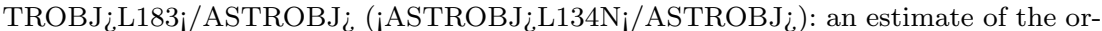
tho/para H\{_2\} ratio. A\&A, 494, 623-636 (2009). DOI 10.1051/0004-6361:200810587

338. Palumbo, M.E., Pendleton, Y.J., Strazzulla, G.: Hydrogen Isotopic Substitution Studies of the 2165 Wavenumber (4.62 Micron) "XCN" Feature Produced by Ion Bombardment. ApJ, 542, 890-893 (2000). DOI 10.1086/317061

339. Parise, B., Belloche, A., Du, F., Güsten, R., Menten, K.M.: Extended emission of $\mathrm{D}_{2} \mathrm{H}^{+}$ in a prestellar core. A\&A, 526, A31 (2011). DOI 10.1051/0004-6361/201015475

340. Parise, B., Castets, A., Herbst, E., Caux, E., Ceccarelli, C., Mukhopadhyay, I., Tielens, A.G.G.M.: First detection of triply-deuterated methanol. A\&A, 416, 159-163 (2004). DOI 10.1051/0004-6361:20034490

341. Parise, B., Caux, E., Castets, A., Ceccarelli, C., Loinard, L., Tielens, A.G.G.M., Bacmann, A., Cazaux, S., Comito, C., Helmich, F., Kahane, C., Schilke, P., van Dishoeck, E., Wakelam, V., Walters, A.: HDO abundance in the envelope of the solar-type protostar IRAS 16293-2422. A\&A, 431, 547-554 (2005). DOI 10.1051/0004-6361:20041899

342. Parise, B., Ceccarelli, C., Tielens, A.G.G.M., Castets, A., Caux, E., Lefloch, B., Maret, S.: Testing grain surface chemistry: a survey of deuterated formaldehyde and methanol in low-mass class 0 protostars. A\&A, 453, 949-958 (2006). DOI 10.1051/0004-6361: 20054476

343. Parise, B., Ceccarelli, C., Tielens, A.G.G.M., Herbst, E., Lefloch, B., Caux, E., Castets, A., Mukhopadhyay, I., Pagani, L., Loinard, L.: Detection of doubly-deuterated methanol in the solar-type protostar IRAS 16293-2422. A\&A, 393, L49-L53 (2002). DOI 10.1051/0004-6361:20021131

344. Peng, R., Yoshida, H., Chamberlin, R.A., Phillips, T.G., Lis, D.C., Gerin, M.: A Comprehensive Survey of Hydrogen Chloride in the Galaxy. ApJ, 723, 218-228 (2010). DOI 10.1088/0004-637X/723/1/218

345. Peng, T.C., Despois, D., Brouillet, N., Parise, B., Baudry, A.: Deuterated methanol in Orion BN/KL. A\&A, 543, A152 (2012). DOI 10.1051/0004-6361/201118310

346. Persson, M.V., Jørgensen, J.K., van Dishoeck, E.F.: Subarcsecond resolution observations of warm water toward three deeply embedded low-mass protostars. A\&A, 541, A39 (2012). DOI 10.1051/0004-6361/201117917

347. Petit, J.M., Mousis, O., Kavelaars, J.J.: Formation Location of Enceladus and Comets from D/H Measurements. In: Lunar and Planetary Institute Science Conference Abstracts, Lunar and Planetary Institute Science Conference Abstracts, vol. 43, p. 1937 (2012)

348. Pickett, H.M., Poynter, R.L., Cohen, E.A., Delitsky, M.L., Pearson, J.C., Müller, H.S.P.: Submillimeter, millimeter and microwave spectral line catalog. Journal of Quantitative Spectroscopy and Radiative Transfer, 60, 883-890 (1998). DOI 10.1016/S0022-4073(98)00091-0

349. Pilling, S., Andrade, D.P.P., da Silveira, E.F., Rothard, H., Domaracka, A., Boduch, P.: Formation of unsaturated hydrocarbons in interstellar ice analogues by cosmic rays. MNRAS, 423, 2209-2221 (2012). DOI 10.1111/j.1365-2966.2012.21031.x

350. Pineda, J.E., Maury, A.J., Fuller, G.A., Testi, L., García-Appadoo, D., Peck, A.B., Villard, E., Corder, S.A., van Kempen, T.A., Turner, J.L., Tachihara, K., Dent, W.: The first ALMA view of IRAS 16293-2422. Direct detection of infall onto source B and high-resolution kinematics of source A. A\&A, 544, L7 (2012). DOI 10.1051/ 0004-6361/201219589

351. Pirronello, V., Liu, C., Roser, J.E., Vidali, G.: Measurements of molecular hydrogen formation on carbonaceous grains. A\&A, 344, 681-686 (1999)

352. Pizzarello, S., Holmes, W.: Nitrogen-containing compounds in two CR2 meteorites: ${ }^{15} \mathrm{~N}$ composition, molecular distribution and precursor molecules. Geochimica et Cosmochimica Acta73, 2150-2162 (2009)

353. Pizzarello, S., Huang, Y.: The deuterium enrichment of individual amino acids in carbonaceous meteorites: A case for the presolar distribution of biomolecule precursors. Geochimica et Cosmochimica Acta69, 599-605 (2005). DOI 10.1016/j.gca.2004.07.031

354. Pizzarello, S., Huang, Y., Becker, L., Poreda, R.J., Nieman, R.A., Cooper, G., Williams, M.: The Organic Content of the Tagish Lake Meteorite. Science 293, 2236-2239 (2001). DOI 10.1126/science.1062614 
355. Pizzarello, S., Zolensky, M., Turk, K.A.: Nonracemic isovaline in the Murchison meteorite: chiral distribution and mineral association. Geochimica et Cosmochimica Acta67, 1589-1595 (2003). DOI 10.1016/S0016-7037(02)01283-8

356. Podio, L., Kamp, I., Flower, D., Howard, C., Sandell, G., Mora, A., Aresu, G., Brittain, S., Dent, W.R.F., Pinte, C., White, G.J.: Herschel/PACS observations of young sources in Taurus: the far-infrared counterpart of optical jets. A\&A, 545, A44 (2012). DOI 10.1051/0004-6361/201219475

357. Pollack, J.B., Hubickyj, O., Bodenheimer, P., Lissauer, J.J., Podolak, M., Greenzweig, Y.: Formation of the Giant Planets by Concurrent Accretion of Solids and Gas. Icarus, 124, 62-85 (1996). DOI 10.1006/icar.1996.0190

358. Pontoppidan, K.M., Salyk, C., Blake, G.A., Käufl, H.U.: Spectrally Resolved Pure Rotational Lines of Water in Protoplanetary Disks. ApJL, 722, L173-L177 (2010). DOI 10.1088/2041-8205/722/2/L173

359. Pontoppidan, K.M., Salyk, C., Blake, G.A., Meijerink, R., Carr, J.S., Najita, J.: A Spitzer Survey of Mid-infrared Molecular Emission from Protoplanetary Disks. I. Detection Rates. ApJ, 720, 887-903 (2010). DOI 10.1088/0004-637X/720/1/887

360. Prasad, S.S., Tarafdar, S.P.: UV radiation field inside dense clouds - Its possible existence and chemical implications. ApJ, 267, 603-609 (1983). DOI 10.1086/160896

361. Preibisch, T., Feigelson, E.D.: The Evolution of X-Ray Emission in Young Stars. ApJS, 160, 390-400 (2005). DOI 10.1086/432094

362. Qi, C., Ho, P.T.P., Wilner, D.J., Takakuwa, S., Hirano, N., Ohashi, N., Bourke, T.L., Zhang, Q., Blake, G.A., Hogerheijde, M., Saito, M., Choi, M., Yang, J.: Imaging the Disk around TW Hydrae with the Submillimeter Array. ApJL, 616, L11-L14 (2004). DOI 10.1086/421063

363. Qi, C., Wilner, D.J., Aikawa, Y., Blake, G.A., Hogerheijde, M.R.: Resolving the Chemistry in the Disk of TW Hydrae. I. Deuterated Species. ApJ, 681, 1396-1407 (2008). DOI $10.1086 / 588516$

364. Quan, D., Herbst, E., Osamura, Y., Roueff, E.: Gas-grain Modeling of Isocyanic Acid (HNCO), Cyanic Acid (HOCN), Fulminic Acid (HCNO), and Isofulminic Acid (HONC) in Assorted Interstellar Environments. ApJ, 725, 2101-2109 (2010). DOI 10.1088/0004-637X/725/2/2101

365. Rafikov, R.R.: Microwave Emission from Spinning Dust in Circumstellar Disks. ApJ, 646, 288-296 (2006). DOI 10.1086/504793

366. Ratajczak, A., Taquet, V., Kahane, C., Ceccarelli, C., Faure, A., Quirico, E.: The puzzling deuteration of methanol in low- to high-mass protostars. A\&A, 528, L13 (2011). DOI 10.1051/0004-6361/201016402

367. Raymond, S.N., O'Brien, D.P., Morbidelli, A., Kaib, N.A.: Building the terrestrial planets: Constrained accretion in the inner Solar System. Icarus, 203, 644-662 (2009). DOI 10.1016/j.icarus.2009.05.016

368. Redman, M.P., Rawlings, J.M.C., Nutter, D.J., Ward-Thompson, D., Williams, D.A.: Molecular gas freeze-out in the pre-stellar core L1689B. MNRAS, 337, L17-L21 (2002). DOI 10.1046/j.1365-8711.2002.06106.x

369. Remijan, A.J., Hollis, J.M., Snyder, L.E., Jewell, P.R., Lovas, F.J.: Methyltriacetylene $\left(\mathrm{CH}_{3} \mathrm{C}_{6} \mathrm{H}\right)$ toward TMC-1: The Largest Detected Symmetric Top. ApJL, 643, L37L40 (2006). DOI 10.1086/504918

370. Remusat, L., Palhol, F., Robert, F., Derenne, S.: Hydrogen Isotopic Composition of Aliphatic Linkages in Carbonaceous Chondrites Insoluble Organic Matter. In: S. Mackwell, E. Stansbery (eds.) 36th Annual Lunar and Planetary Science Conference, Lunar and Planetary Institute Science Conference Abstracts, vol. 36, p. 1350 (2005)

371. Remusat, L., Robert, F., Meibom, A., Mostefaoui, S., Delpoux, O., Binet, L., Gourier, D., Derenne, S.: Proto-Planetary Disk Chemistry Recorded by D-Rich Organic Radicals in Carbonaceous Chondrites. ApJ, 698, 2087-2092 (2009). DOI 10.1088/0004-637X/698/2/2087

372. Requena-Torres, M.A., Marcelino, N., Jiménez-Serra, I., Martín-Pintado, J., Martín, S. Mauersberger, R.: Organic Chemistry in the Dark Clouds L1448 and L183: A Unique Grain Mantle Composition. ApJL, 655, L37-L40 (2007). DOI 10.1086/511677

373. Riaz, B., Honda, M., Campins, H., Micela, G., Guarcello, M.G., Gledhill, T., Hough, J., Martín, E.L.: The radial distribution of dust species in young brown dwarf discs. MNRAS, 420, 2603-2624 (2012). DOI 10.1111/j.1365-2966.2011.20233.x 
374. Ricci, L., Testi, L., Maddison, S.T., Wilner, D.J.: Fomalhaut debris disk emission at 7 millimeters: constraints on the collisional models of planetesimals. A\&A, 539, L6 (2012). DOI 10.1051/0004-6361/201118524

375. Robert, F.: The D/H Ratio in Chondrites. Space Science Reviews, 106, 87-101 (2003). DOI 10.1023/A:1024629402715

376. Robert, F., Derenne, S.: The Molecular Structure and Isotopic Compositions of the Insoluble Organic Matter in Chondrites. Meteoritics and Planetary Science Supplement 41, 5259 (2006)

377. Roberts, H., Herbst, E., Millar, T.J.: Enhanced Deuterium Fractionation in Dense Interstellar Cores Resulting from Multiply Deuterated $\mathrm{H}^{+}{ }_{3}$. ApJL, 591, L41-L44 (2003). DOI 10.1086/376962

378. Roberts, J.F., Rawlings, J.M.C., Viti, S., Williams, D.A.: Desorption from interstellar ices. MNRAS, 382, 733-742 (2007). DOI 10.1111/j.1365-2966.2007.12402.x

379. Robitaille, T.P., Whitney, B.A., Indebetouw, R., Wood, K., Denzmore, P.: Interpreting Spectral Energy Distributions from Young Stellar Objects. I. A Grid of 200,000 YSO Model SEDs. ApJS, 167, 256-285 (2006). DOI 10.1086/508424

380. Rodríguez, L.F., Loinard, L., D'Alessio, P., Wilner, D.J., Ho, P.T.P.: IRAS 162932422B: A Compact, Possibly Isolated Protoplanetary Disk in a Class 0 Object. ApJL, 621, L133-L136 (2005). DOI 10.1086/429223

381. Romanzin, C., Ioppolo, S., Cuppen, H.M., van Dishoeck, E.F., Linnartz, H.: Water formation by surface O3 hydrogenation. JChPh, 134(8), 084,504 (2011). DOI 10. $1063 / 1.3532087$

382. Sakai, N., Ikeda, M., Morita, M., Sakai, T., Takano, S., Osamura, Y., Yamamoto, S.: Production Pathways of CCS and CCCS Inferred from Their ${ }^{13} \mathrm{C}$ Isotopic Species. ApJ, 663, 1174-1179 (2007). DOI 10.1086/518595

383. Sakai, N., Sakai, T., Hirota, T., Yamamoto, S.: Abundant Carbon-Chain Molecules toward the Low-Mass Protostar IRAS 04368+2557 in L1527. ApJ, 672, 371-381 (2008). DOI $10.1086 / 523635$

384. Sakai, N., Saruwatari, O., Sakai, T., Takano, S., Yamamoto, S.: Abundance anomaly of the ${ }^{13} \mathrm{C}$ species of CCH. A\&A, 512, A31 (2010). DOI 10.1051/0004-6361/200913098

385. Sakai, N., Shiino, T., Hirota, T., Sakai, T., Yamamoto, S.: Long Carbon-chain Molecules and Their Anions in the Starless Core, Lupus-1A. ApJL, 718, L49-L52 (2010). DOI 10.1088/2041-8205/718/2/L49

386. Salyk, C., Pontoppidan, K.M., Blake, G.A., Lahuis, F., van Dishoeck, E.F., Evans II, N.J.: $\mathrm{H}_{2} \mathrm{O}$ and $\mathrm{OH}$ Gas in the Terrestrial Planet-forming Zones of Protoplanetary Disks. ApJL, 676, L49-L52 (2008). DOI 10.1086/586894

387. Salyk, C., Pontoppidan, K.M., Blake, G.A., Najita, J.R., Carr, J.S.: A Spitzer Survey of Mid-infrared Molecular Emission from Protoplanetary Disks. II. Correlations and Local Thermal Equilibrium Models. ApJ, 731, 130 (2011). DOI 10.1088/0004-637X/ $731 / 2 / 130$

388. Santangelo, G., Nisini, B., Giannini, T., Antoniucci, S., Vasta, M., Codella, C., Lorenzani, A., Tafalla, M., Liseau, R., van Dishoeck, E.F., Kristensen, L.E.: The Herschel HIFI water line survey in the low-mass proto-stellar outflow L1448. A\&A, 538, A45 (2012). DOI 10.1051/0004-6361/201118113

389. Sargent, B.A., Forrest, W.J., Tayrien, C., McClure, M.K., Watson, D.M., Sloan, G.C., Li, A., Manoj, P., Bohac, C.J., Furlan, E., Kim, K.H., Green, J.D.: Dust Processing and Grain Growth in Protoplanetary Disks in the Taurus-Auriga Star-Forming Region. ApJS, 182, 477-508 (2009). DOI 10.1088/0067-0049/182/2/477

390. Schaller, E.L., Brown, M.E.: Volatile Loss and Retention on Kuiper Belt Objects. In AAS/Division for Planetary Sciences Meeting Abstracts \#39, Bulletin of the American Astronomical Society, vol. 39, p. 511 (2007)

391. Schöier, F.L., Jørgensen, J.K., van Dishoeck, E.F., Blake, G.A.: Does IRAS 162932422 have a hot core? Chemical inventory and abundance changes in its protostellar environment. A\&A, 390, 1001-1021 (2002). DOI 10.1051/0004-6361:20020756

392. Schöier, F.L., van der Tak, F.F.S., van Dishoeck, E.F., Black, J.H.: An atomic and molecular database for analysis of submillimetre line observations. A\&A, 432, 369379 (2005). DOI 10.1051/0004-6361:20041729

393. Schopf, J.W., Kudryavtsev, A.B., Agresti, D.G., Wdowiak, T.J., Czaja, A.D.: LaserRaman imagery of Earth's earliest fossils. Nature, 416, 73-76 (2002) 
394. Schräpler, R., Blum, J., Seizinger, A., Kley, W.: The physics of protoplanetesimal dust agglomerates. VII The low-velocity collision behavior of large dust agglomerates. ArXiv e-prints (2012)

395. Semenov, D.A.: Chemical Evolution of a Protoplanetary Disk. In: IAU Symposium, IAU Symposium, vol. 280, pp. 114-126 (2011). DOI 10.1017/S1743921311024914

396. Shen, C.J., Greenberg, J.M., Schutte, W.A., van Dishoeck, E.F.: Cosmic ray induced explosive chemical desorption in dense clouds. A\&A, 415, 203-215 (2004). DOI 10.1051/0004-6361:20031669

397. Shimajiri, Y., Takahashi, S., Takakuwa, S., Saito, M., Kawabe, R.: Millimeter- and Submillimeter-Wave Observations of the OMC-2/3 Region. II. Observational Evidence for Outflow-triggered Star Formation in the OMC-2 FIR 3/4 Region. ApJ, 683, 255266 (2008). DOI 10.1086/588629

398. Shu, F.H.: Self-similar collapse of isothermal spheres and star formation. ApJ, 214, 488-497 (1977). DOI 10.1086/155274

399. Shu, F.H., Adams, F.C., Lizano, S.: Star formation in molecular clouds - Observation and theory. ARA\&A, 25, 23-81 (1987). DOI 10.1146/annurev.aa.25.090187.000323

400. Sicilia, D., Ioppolo, S., Vindigni, T., Baratta, G.A., Palumbo, M.E.: Nitrogen oxides and carbon chain oxides formed after ion irradiation of $\mathrm{CO}: \mathrm{N}_{2}$ ice mixtures. A\&A, 543, A155 (2012). DOI 10.1051/0004-6361/201219390

401. Siebenmorgen, R., Heymann, F.: Polycyclic aromatic hydrocarbons in protoplanetary disks: emission and X-ray destruction. A\&A, 543, A25 (2012). DOI 10.1051/ 0004-6361/201219039

402. Siebenmorgen, R., Krügel, E.: The destruction and survival of polycyclic aromatic hydrocarbons in the disks of $\mathrm{T}$ Tauri stars. A\&A, 511, A6 (2010). DOI 10.1051/ 0004-6361/200912035

403. Skrzypczak, A., Binet, L., Gourier, D., Derenne, S., Robert, F.: On the Controversial Biogenicity of the Organic Matter in the Oldest Archean Cherts: Can Electron Paramagnetic Resonance Provide Clues? In: S. Mackwell, E. Stansbery (eds.) Lunar and Planetary Institute Science Conference Abstracts, Lunar and Planetary Institute Science Conference Abstracts, vol. 34, p. 1677 (2003)

404. Snyder, L.E., Hollis, J.M., Jewell, P.R., Lovas, F.J., Remijan, A.: Confirmation of Interstellar Methylcyanodiacetylene $\left(\mathrm{CH}_{3} \mathrm{C}_{5} \mathrm{~N}\right)$. ApJ, 647, 412-417 (2006). DOI 10. 1086/505323

405. Spitzer, L.: Physical processes in the interstellar medium (1978)

406. Stäuber, P., Doty, S.D., van Dishoeck, E.F., Benz, A.O.: X-ray chemistry in the envelopes around young stellar objects. A\&A, 440, 949-966 (2005). DOI 10.1051/ 0004-6361:20052889

407. Sturm, B., Bouwman, J., Henning, T., Evans, N.J., Acke, B., Mulders, G.D., Waters, L.B.F.M., van Dishoeck, E.F., Meeus, G., Green, J.D., Augereau, J.C., Olofsson, J., Salyk, C., Najita, J., Herczeg, G.J., van Kempen, T.A., Kristensen, L.E., Dominik, C., Carr, J.S., Waelkens, C., Bergin, E., Blake, G.A., Brown, J.M., Chen, J.H., Cieza, L., Dunham, M.M., Glassgold, A., Güdel, M., Harvey, P.M., Hogerheijde, M.R., Jaffe, D., Jørgensen, J.K., Kim, H.J., Knez, C., Lacy, J.H., Lee, J.E., Maret, S., Meijerink, R., Merín, B., Mundy, L., Pontoppidan, K.M., Visser, R., Yıldız, U.A.: First results of the Herschel key program "Dust, Ice and Gas In Time" (DIGIT): Dust and gas spectroscopy of HD 100546. A\&A, 518, L129 (2010). DOI 10.1051/0004-6361/201014674

408. Tafalla, M., Myers, P.C., Caselli, P., Walmsley, C.M.: On the internal structure of starless cores. I. Physical conditions and the distribution of $\mathrm{CO}, \mathrm{CS}, \mathrm{N}_{2} \mathrm{H}^{+}$, and $\mathrm{NH}_{3}$ in L1498 and L1517B. A\&A, 416, 191-212 (2004). DOI 10.1051/0004-6361:20031704

409. Tafalla, M., Santiago-García, J., Myers, P.C., Caselli, P., Walmsley, C.M., Crapsi, A.: On the internal structure of starless cores. II. A molecular survey of L1498 and L1517B. A\&A, 455, 577-593 (2006). DOI 10.1051/0004-6361:20065311

410. Taquet, V., Ceccarelli, C., Kahane, C.: Formaldehyde and Methanol Deuteration in Protostars: Fossils from a Past Fast High-density Pre-collapse Phase. ApJL, 748, L3 (2012). DOI 10.1088/2041-8205/748/1/L3

411. Taquet, V., Ceccarelli, C., Kahane, C.: Multilayer modeling of porous grain surface chemistry. I. The GRAINOBLE model. A\&A, 538, A42 (2012). DOI 10.1051/ 0004-6361/201117802 
412. Taquet, V., Lopez-Sepulcre, A., Ceccarelli, C., Kahane, C.: Arcsecond resolution observations of deuterated water towards low-mass protostar. A\&A, in print, A42 (2012). DOI 10.1051/0004-6361/201117812

413. Taquet, V., Peters, P., Kahane, C., Ceccarelli, C., Lopez-Sepulcre, A., Toubin, C., Duflot, D., Wiesenfeld, L.: Modelling of deuterated water ice formation. A\&A, in print, A42 (2012). DOI 10.1051/0004-6361/201117802

414. Tatulli, E., Isella, A., Natta, A., Testi, L., Marconi, A., Malbet, F., Stee, P., Petrov, R.G., Millour, F., Chelli, A., Duvert, G., Antonelli, P., Beckmann, U., Bresson, Y., Dugué, M., Gennari, S., Glück, L., Kern, P., Lagarde, S., Le Coarer, E., Lisi, F., Perraut, K., Puget, P., Rantakyrö, F., Robbe-Dubois, S., Roussel, A., Weigelt, G. Zins, G., Accardo, M., Acke, B., Agabi, K., Altariba, E., Arezki, B., Aristidi, E., Baffa, C., Behrend, J., Blöcker, T., Bonhomme, S., Busoni, S., Cassaing, F., Clausse, J.M., Colin, J., Connot, C., Delboulbé, A., Domiciano de Souza, A., Driebe, T., Feautrier, P., Ferruzzi, D., Forveille, T., Fossat, E., Foy, R., Fraix-Burnet, D., Gallardo, A., Giani, E., Gil, C., Glentzlin, A., Heiden, M., Heininger, M., Hernandez Utrera, O., Hofmann, K.H., Kamm, D., Kiekebusch, M., Kraus, S., Le Contel, D., Le Contel, J.M., Lesourd, T., Lopez, B., Lopez, M., Magnard, Y., Mars, G., Martinot-Lagarde, G., Mathias, P., Mège, P., Monin, J.L., Mouillet, D., Mourard, D., Nussbaum, E., Ohnaka, K., Pacheco, J., Perrier, C., Rabbia, Y., Rebattu, S., Reynaud, F., Richichi, A., Robini, A., Sacchettini, M., Schertl, D., Schöller, M., Solscheid, W., Spang, A., Stefanini, P., Tallon, M., Tallon-Bosc, I., Tasso, D., Vakili, F., von der Lühe, O., Valtier, J.C., Vannier, M., Ventura, N.: Constraining the wind launching region in Herbig Ae stars: AMBER/VLTI spectroscopy of HD 104237. A\&A, 464, 55-58 (2007). DOI 10.1051/0004-6361:20065719

415. Terada, H., Tokunaga, A.T., Kobayashi, N., Takato, N., Hayano, Y., Takami, H.: Detection of Water Ice in Edge-on Protoplanetary Disks: HK Tauri B and HV Tauri C. ApJ, 667, 303-307 (2007). DOI 10.1086/520951

416. Testi, L., Natta, A., Shepherd, D.S., Wilner, D.J.: Large grains in the disk of CQ Tau. A\&A, 403, 323-328 (2003). DOI 10.1051/0004-6361:20030362

417. Thi, W.F., Mathews, G., Ménard, F., Woitke, P., Meeus, G., Riviere-Marichalar, P., Pinte, C., Howard, C.D., Roberge, A., Sandell, G., Pascucci, I., Riaz, B., Grady, C.A., Dent, W.R.F., Kamp, I., Duchêne, G., Augereau, J.C., Pantin, E., Vandenbussche, B., Tilling, I., Williams, J.P., Eiroa, C., Barrado, D., Alacid, J.M., Andrews, S., Ardila, D.R., Aresu, G., Brittain, S., Ciardi, D.R., Danchi, W., Fedele, D., de Gregorio-Monsalvo, I., Heras, A., Huelamo, N., Krivov, A., Lebreton, J., Liseau, R., Martin-Zaidi, C., Mendigutía, I., Montesinos, B., Mora, A., Morales-Calderon, M., Nomura, H., Phillips, N., Podio, L., Poelman, D.R., Ramsay, S., Rice, K., Solano, E., Walker, H., White, G.J., Wright, G.: Herschel-PACS observation of the 10 Myr old T Tauri disk TW Hya. Constraining the disk gas mass. A\&A, 518, L125 (2010). DOI 10.1051/0004-6361/201014578

418. Thi, W.F., van Zadelhoff, G.J., van Dishoeck, E.F.: Organic molecules in protoplanetary disks around T Tauri and Herbig Ae stars. A\&A, 425, 955-972 (2004). DOI 10.1051/0004-6361:200400026

419. Thi, W.F., Woitke, P., Kamp, I.: Warm non-equilibrium gas phase chemistry as a possible origin of high $\mathrm{HDO} / \mathrm{H}_{2} \mathrm{O}$ ratios in hot and dense gases: application to inner protoplanetary discs. MNRAS, 407, 232-246 (2010). DOI 10.1111/j.1365-2966.2009. 16162.x

420. Thomas, K.L., Blanford, G.E., Keller, L.P., Klock, W., McKay, D.S.: Carbon abundance and silicate mineralogy of anhydrous interplanetary dust particles. Geochimica et Cosmochimica Acta57, 1551-1566 (1993). DOI 10.1016/0016-7037(93)90012-L

421. Tielens, A.G.G.M.: Surface chemistry of deuterated molecules. A\&A, 119, 177-184 (1983)

422. Tielens, A.G.G.M.: The Physics and Chemistry of the Interstellar Medium (2005)

423. Troland, T.H., Crutcher, R.M.: Magnetic Fields in Dark Cloud Cores: Arecibo $\mathrm{OH}$ Zeeman Observations. ApJ, 680, 457-465 (2008). DOI 10.1086/587546

424. Troscompt, N., Faure, A., Maret, S., Ceccarelli, C., Hily-Blant, P., Wiesenfeld, L.: Constraining the ortho-to-para ratio of $\mathrm{H}_{2}$ with anomalous $\mathrm{H}_{-} 2 \mathrm{CO}$ absorption. A\&A, 506, 1243-1247 (2009). DOI 10.1051/0004-6361/200912770 
425. van der Tak, F.F.S., Caselli, P., Ceccarelli, C.: Line profiles of molecular ions toward the pre-stellar core LDN 1544. A\&A, 439, 195-203 (2005). DOI 10.1051/0004-6361: 20052792

426. van der Tak, F.F.S., Schilke, P., Müller, H.S.P., Lis, D.C., Phillips, T.G., Gerin, M., Roueff, E.: Triply deuterated ammonia in NGC 1333. A\&A, 388, L53-L56 (2002). DOI 10.1051/0004-6361:20020647

427. van Dishoeck, E.F., Kristensen, L.E., Benz, A.O., Bergin, E.A., Caselli, P., Cernicharo, J., Herpin, F., Hogerheijde, M.R., Johnstone, D., Liseau, R., Nisini, B., Shipman, R., Tafalla, M., van der Tak, F., Wyrowski, F., Aikawa, Y., Bachiller, R., Baudry, A., Benedettini, M., Bjerkeli, P., Blake, G.A., Bontemps, S., Braine, J., Brinch, C., Bruderer, S., Chavarría, L., Codella, C., Daniel, F., de Graauw, T., Deul, E., di Giorgio, A.M., Dominik, C., Doty, S.D., Dubernet, M.L., Encrenaz, P., Feuchtgruber, H., Fich, M., Frieswijk, W., Fuente, A., Giannini, T., Goicoechea, J.R., Helmich, F.P., Herczeg, G.J., Jacq, T., Jørgensen, J.K., Karska, A., Kaufman, M.J., Keto, E., Larsson, B. Lefloch, B., Lis, D., Marseille, M., McCoey, C., Melnick, G., Neufeld, D., Olberg, M., Pagani, L., Panić, O., Parise, B., Pearson, J.C., Plume, R., Risacher, C., Salter, D., Santiago-García, J., Saraceno, P., Stäuber, P., van Kempen, T.A., Visser, R., Viti, S., Walmsley, M., Wampfler, S.F., Yıldız, U.A.: Water in Star-forming Regions with the Herschel Space Observatory (WISH). I. Overview of Key Program and First Results. PASP, 123, 138-170 (2011). DOI 10.1086/658676

428. van Dishoeck, E.F., Thi, W.F., van Zadelhoff, G.J.: Detection of $\mathrm{DCO}^{+}$in a circumstellar disk. A\&A, 400, L1-L4 (2003). DOI 10.1051/0004-6361:20030091

429. van Kempen, T.A., van Dishoeck, E.F., Güsten, R., Kristensen, L.E., Schilke, P., Hogerheijde, M.R., Boland, W., Menten, K.M., Wyrowski, F.: APEX-CHAMP ${ }^{+}$high-J $\mathrm{CO}$ observations of low-mass young stellar objects. II. Distribution and origin of warm molecular gas. A\&A, 507, 1425-1442 (2009). DOI 10.1051/0004-6361/200912507

430. van Zadelhoff, G.J., van Dishoeck, E.F., Thi, W.F., Blake, G.A.: Submillimeter lines from circumstellar disks around pre-main sequence stars. A\&A, 377, 566-580 (2001). DOI 10.1051/0004-6361:20011137

431. Vasta, M., Codella, C., Lorenzani, A., Santangelo, G., Nisini, B., Giannini, T., Tafalla, M., Liseau, R., van Dishoeck, E.F., Kristensen, L.: Water emission from the chemically rich outflow L1157. A\&A, 537, A98 (2012). DOI 10.1051/0004-6361/201118201

432. Vastel, C., Ceccarelli, C., Caux, E., Coutens, A., Cernicharo, J., Bottinelli, S., Demyk, K., Faure, A., Wiesenfeld, L., Scribano, Y., Bacmann, A., Hily-Blant, P., Maret, S., Walters, A., Bergin, E.A., Blake, G.A., Castets, A., Crimier, N., Dominik, C., Encrenaz, P., Gérin, M., Hennebelle, P., Kahane, C., Klotz, A., Melnick, G., Pagani, L., Parise, B., Schilke, P., Wakelam, V., Baudry, A., Bell, T., Benedettini, M., Boogert, A., Cabrit, S., Caselli, P., Codella, C., Comito, C., Falgarone, E., Fuente, A., Goldsmith, P.F., Helmich, F., Henning, T., Herbst, E., Jacq, T., Kama, M., Langer, W., Lefloch, B., Lis, D., Lord, S., Lorenzani, A., Neufeld, D., Nisini, B., Pacheco, S., Pearson, J., Phillips, T., Salez, M., Saraceno, P., Schuster, K., Tielens, X., van der Tak, F., van der Wiel, M.H.D., Viti, S., Wyrowski, F., Yorke, H., Cais, P., Krieg, J.M., Olberg, M., Ravera, L.: Ortho-to-para ratio of interstellar heavy water. A\&A, 521, L31 (2010). DOI 10.1051/0004-6361/201015101

433. Vastel, C., Phillips, T.G., Ceccarelli, C., Pearson, J.: First Detection of Doubly Deuterated Hydrogen Sulfide. ApJL, 593, L97-L100 (2003). DOI 10.1086/378261

434. Vastel, C., Phillips, T.G., Yoshida, H.: Detection of $\mathrm{D}_{2} \mathrm{H}^{+}$in the Dense Interstellar Medium. ApJL, 606, L127-L130 (2004). DOI 10.1086/421265

435. Vasyunin, A.I., Semenov, D., Henning, T., Wakelam, V., Herbst, E., Sobolev, A.M.: Chemistry in Protoplanetary Disks: A Sensitivity Analysis. ApJ, 672, 629-641 (2008). DOI 10.1086/523887

436. Vasyunin, A.I., Wiebe, D.S., Birnstiel, T., Zhukovska, S., Henning, T., Dullemond, C.P.: Impact of Grain Evolution on the Chemical Structure of Protoplanetary Disks. ApJ, 727, 76 (2011). DOI 10.1088/0004-637X/727/2/76

437. Villeneuve, J., Chaussidon, M., Libourel, G.: Homogeneous Distribution of ${ }^{26} \mathrm{Al}$ in the Solar System from the Mg Isotopic Composition of Chondrules. Science 325, 985(2009). DOI 10.1126/science.1173907 
438. Visser, R., Doty, S.D., van Dishoeck, E.F.: The chemical history of molecules in circumstellar disks. II. Gas-phase species. A\&A, 534, A132 (2011). DOI 10.1051/0004-6361/ 201117249

439. Visser, R., Geers, V.C., Dullemond, C.P., Augereau, J.C., Pontoppidan, K.M., van Dishoeck, E.F.: PAH chemistry and IR emission from circumstellar disks. A\&A, 466, 229-241 (2007). DOI 10.1051/0004-6361:20066829

440. Visser, R., Kristensen, L.E., Bruderer, S., van Dishoeck, E.F., Herczeg, G.J., Brinch C., Doty, S.D., Harsono, D., Wolfire, M.G.: Modelling Herschel observations of hot molecular gas emission from embedded low-mass protostars. A\&A, 537, A55 (2012). DOI 10.1051/0004-6361/201117109

441. Visser, R., van Dishoeck, E.F., Doty, S.D., Dullemond, C.P.: The chemical history of molecules in circumstellar disks. I. Ices. A\&A, 495, 881-897 (2009). DOI 10.1051/ 0004-6361/200810846

442. Viti, S., Collings, M.P., Dever, J.W., McCoustra, M.R.S., Williams, D.A.: Evaporation of ices near massive stars: models based on laboratory temperature programmed desorption data. MNRAS, 354, 1141-1145 (2004). DOI 10.1111/j.1365-2966.2004.08273.x

443. Vorobyov, E.I.: Embedded Protostellar Disks Around (Sub-)Solar Stars. II. Disk Masses, Sizes, Densities, Temperatures, and the Planet Formation Perspective. ApJ, 729, 146 (2011). DOI 10.1088/0004-637X/729/2/146

444. Wakelam, V., Herbst, E.: Polycyclic Aromatic Hydrocarbons in Dense Cloud Chemistry. ApJ, 680, 371-383 (2008). DOI 10.1086/587734

445. Wakelam, V., Herbst, E., Loison, J.C., Smith, I.W.M., Chandrasekaran, V., Pavone, B., Adams, N.G., Bacchus-Montabonel, M.C., Bergeat, A., Béroff, K., Bierbaum, V.M., Chabot, M., Dalgarno, A., van Dishoeck, E.F., Faure, A., Geppert, W.D., Gerlich, D., Galli, D., Hébrard, E., Hersant, F., Hickson, K.M., Honvault, P., Klippenstein, S.J., Le Picard, S., Nyman, G., Pernot, P., Schlemmer, S., Selsis, F., Sims, I.R., Talbi, D., Tennyson, J., Troe, J., Wester, R., Wiesenfeld, L.: A KInetic Database for Astrochemistry (KIDA). ApJS, 199, 21 (2012). DOI 10.1088/0067-0049/199/1/21

446. Wakelam, V., Herbst, E., Selsis, F.: The effect of uncertainties on chemical models of dark clouds. A\&A, 451, 551-562 (2006). DOI 10.1051/0004-6361:20054682

447. Walmsley, C.M., Jewell, P.R., Snyder, L.E., Winnewisser, G.: Detection of interstellar methyldiacetylene $(\mathrm{CH} 3 \mathrm{C} 4 \mathrm{H})$ in the dark dust cloud TMC 1. A\&A, 134, L11-L14 (1984)

448. Walsh, C., Nomura, H., Millar, T.J., Aikawa, Y.: Chemical Processes in Protoplanetary Disks. II. On the Importance of Photochemistry and X-Ray Ionization. ApJ, 747, 114 (2012). DOI 10.1088/0004-637X/747/2/114

449. Ward-Thompson, D., Motte, F., Andre, P.: The initial conditions of isolated star formation - III. Millimetre continuum mapping of pre-stellar cores. MNRAS, 305, 143-150 (1999). DOI 10.1046/j.1365-8711.1999.02412.x

450. Ward-Thompson, D., Scott, P.F., Hills, R.E., Andre, P.: A Submillimetre Continuum Survey of Pre Protostellar Cores. MNRAS, 268, 276 (1994)

451. Wardle, M.: Star Formation and the Hall Effect. Ap\&SS, 292, 317-323 (2004). DOI 10.1023/B:ASTR.0000045033.80068.1f

452. Watanabe, N., Kouchi, A.: Efficient Formation of Formaldehyde and Methanol by the Addition of Hydrogen Atoms to $\mathrm{CO}$ in $\mathrm{H}_{2} \mathrm{O}-\mathrm{CO}$ Ice at $10 \mathrm{~K}$. ApJL, 571, L173-L176 (2002). DOI 10.1086/341412

453. Watson, W.D.: Ion-Molecule Reactions, Molecule Formation, and Hydrogen-Isotope Exchange in Dense Interstellar Clouds. ApJ, 188, 35-42 (1974). DOI 10.1086/152681

454. Weidenschilling, S.J.: Aerodynamics of solid bodies in the solar nebula. MNRAS, 180, 57-70 (1977)

455. Whittet, D.C.B.: Oxygen Depletion in the Interstellar Medium: Implications for Grain Models and the Distribution of Elemental Oxygen. ApJ, 710, 1009-1016 (2010). DOI 10.1088/0004-637X/710/2/1009

456. Whittet, D.C.B., Cook, A.M., Herbst, E., Chiar, J.E., Shenoy, S.S.: Observational Constraints on Methanol Production in Interstellar and Preplanetary Ices. ApJ, 742 28 (2011). DOI 10.1088/0004-637X/742/1/28

457. Whittet, D.C.B., Duley, W.W.: Carbon monoxide frosts in the interstellar medium. A\&ARev, 2, 167-189 (1991). DOI 10.1007/BF00872766 
458. Wienen, M., Wyrowski, F., Schuller, F., Menten, K.M., Walmsley, C.M., Bronfman, L., Motte, F.: Ammonia from cold high-mass clumps discovered in the inner Galactic disk by the ATLASGAL survey. A\&A, 544, A146 (2012). DOI 10.1051/0004-6361/ 201118107

459. Willacy, K., Langer, W., Allen, M., Bryden, G.: Turbulence-driven Diffusion in Protoplanetary Disks: Chemical Effects in the Outer Regions. ApJ, 644, 1202-1213 (2006). DOI 10.1086/503702

460. Willacy, K., Langer, W.D.: The Importance of Photoprocessing in Protoplanetary Disks. ApJ, 544, 903-920 (2000). DOI 10.1086/317236

461. Willacy, K., Langer, W.D., Velusamy, T.: Dust Emission and Molecular Depletion in L1498. ApJL, 507, L171-L175 (1998). DOI 10.1086/311695

462. Willacy, K., Millar, T.J.: Desorption processes and the deuterium fractionation in molecular clouds. MNRAS, 298, 562-568 (1998). DOI 10.1046/j.1365-8711.1998.01648. $\mathrm{X}$

463. Williams, J.P., Cieza, L.A.: Protoplanetary Disks and Their Evolution. ARA\&A, 49 , 67-117 (2011). DOI 10.1146/annurev-astro-081710-102548

464. Wilner, D.J., D’Alessio, P., Calvet, N., Claussen, M.J., Hartmann, L.: Toward Planetesimals in the Disk around TW Hydrae: 3.5 Centimeter Dust Emission. ApJL, 626 , L109-L112 (2005). DOI 10.1086/431757

465. Windmark, F., Birnstiel, T., Ormel, C.W., Dullemond, C.P.: Breaking through: The effects of a velocity distribution on barriers to dust growth. A\&A, 544, L16 (2012). DOI 10.1051/0004-6361/201220004

466. Wirström, E.S., Charnley, S.B., Cordiner, M.A., Milam, S.N.: Isotopic Anomalies in Primitive Solar System Matter: Spin-state-dependent Fractionation of Nitrogen and Deuterium in Interstellar Clouds. ApJL, 757, L11 (2012). DOI 10.1088/2041-8205/ $757 / 1 / \mathrm{L} 11$

467. Woitke, P., Pinte, C., Tilling, I., Ménard, F., Kamp, I., Thi, W.F., Duchêne, G., Augereau, J.C.: Continuum and line modelling of discs around young stars - I. 300000 disc models for HERSCHEL/GASPS. MNRAS, 405, L26-L30 (2010). DOI $10.1111 / \mathrm{j} .1745-3933.2010 .00852 . \mathrm{x}$

468. Wood, K., Lada, C.J., Bjorkman, J.E., Kenyon, S.J., Whitney, B., Wolff, M.J.: Infrared Signatures of Protoplanetary Disk Evolution. ApJ, 567, 1183-1191 (2002). DOI $10.1086 / 338662$

469. Woodall, J., Agúndez, M., Markwick-Kemper, A.J., Millar, T.J.: The UMIST database for astrochemistry 2006. A\&A, 466, 1197-1204 (2007). DOI 10.1051/0004-6361: 20064981

470. Wooden, D.H., Harker, D.E., Woodward, C.E., Butner, H.M., Koike, C., Witteborn, F.C., McMurtry, C.W.: Silicate Mineralogy of the Dust in the Inner Coma of Comet C/1995 01 (Hale-Bopp) Pre- and Postperihelion. ApJ, 517, 1034-1058 (1999). DOI $10.1086 / 307206$

471. Wooden, D.H., Woodward, C.E., Harker, D.E.: Discovery of Crystalline Silicates in Comet C/2001 Q4 (NEAT). ApJL, 612, L77-L80 (2004). DOI 10.1086/424593

472. Wootten, A.: The Duplicity of IRAS 16293-2422: A Protobinary Star? ApJ, 337, 858 (1989). DOI 10.1086/167156

473. Wootten, A., Snell, R., Glassgold, A.E.: The determination of electron abundances in interstellar clouds. ApJ, 234, 876-880 (1979). DOI 10.1086/157569

474. Wouterloot, J.G.A., Henkel, C., Brand, J., Davis, G.R.: Galactic interstellar ${ }^{18} \mathrm{O} /\left\{{ }^{\wedge} 17\right\} \mathrm{O}$ ratios - a radial gradient? A\&A, 487, 237-246 (2008). DOI 10.1051/ 0004-6361:20078156

475. Wyatt, M.C.: Evolution of Debris Disks. ARA\&A, 46, 339-383 (2008). DOI 10.1146/ annurev.astro.45.051806.110525

476. Xie, T., Allen, M., Langer, W.D.: Turbulent Diffusion and Its Effects on the Chemistry of Molecular Clouds. ApJ, 440, 674 (1995). DOI 10.1086/175305

477. Yamaguchi, T., Takano, S., Sakai, N., Takeshi Sakai Sheng-Yuan, T.L., Su, Y.N., Hirano, N., Takakuwa, S., Aikawa, Y., Nomura, H., Yamamoto, S.: Detection of Phosphorus Nitride in the Lynds 1157 B1 Shocked Region. PASJ, 63, L37-L41 (2011)

478. Yang, L., Ciesla, F.J.: The effects of disk building on the distributions of refractory materials in the solar nebula. Meteoritics and Planetary Science 47, 99-119 (2012). DOI 10.1111/j.1945-5100.2011.01315.x 
479. Yıldız, U.A., Kristensen, L.E., van Dishoeck, E.F., Belloche, A., van Kempen, T.A., Hogerheijde, M.R., Güsten, R., van der Marel, N.: APEX-CHAMP ${ }^{+}$high-J CO observations of low-mass young stellar objects. III. NGC 1333 IRAS 4A/4B envelope, outflow, and ultraviolet heating. A\&A, 542, A86 (2012). DOI 10.1051/0004-6361/ 201118368

480. Young, E.D., Gounelle, M., Smith, R.L., Morris, M.R., Pontoppidan, K.M.: Astronomical Oxygen Isotopic Evidence for Supernova Enrichment of the Solar System Birth Environment by Propagating Star Formation. ApJ, 729, 43 (2011). DOI 10.1088/0004-637X/729/1/43

481. Zolensky, M., Nakamura-Messenger, K., Rietmeijer, F., Leroux, H., Mikouchi, T., Ohsumi, K., Simon, S., Grossman, L., Stephan, T., Weisberg, M., Velbel, M., Zega, T., Stroud, R., Tomeoka, K., Ohnishi, I., Tomioka, N., Nakamura, T., Matrajt, G., Joswiak, D., Brownlee, D., Langenhorst, F., Krot, A., Kearsley, A., Ishii, H., Graham, G., Dai, Z.R., Chi, M., Bradley, J., Hagiya, K., Gounelle, M., Bridges, J.: Comparing Wild 2 particles to chondrites and IDPs. Meteoritics and Planetary Science 43, 261-272 (2008). DOI 10.1111/j.1945-5100.2008.tb00621.x

482. Zsom, A., Ormel, C.W., Dullemond, C.P., Henning, T.: The outcome of protoplanetary dust growth: pebbles, boulders, or planetesimals?. III. Sedimentation driven coagulation inside the snowline. A\&A, 534, A73 (2011). DOI 10.1051/0004-6361/201116515 